\title{
Rigidity in equivariant stable homotopy theory
}

\author{
IRAKLI PATCHKORIA
}

\begin{abstract}
For any finite group $G$, we show that the 2 -local $G$-equivariant stable homotopy category, indexed on a complete $G$-universe, has a unique equivariant model in the sense of Quillen model categories. This means that the suspension functor, homotopy cofiber sequences and the stable Burnside category determine all "higherorder structure" of the 2-local $G$-equivariant stable homotopy category, such as the equivariant homotopy types of function $G$-spaces. Our result can be seen as an equivariant version of Schwede's rigidity theorem at the prime 2.
\end{abstract}

55P42, 55P91; 18G55

\section{Introduction}

One of the most difficult problems of algebraic topology is to calculate the stable homotopy groups of spheres. There has been extensive research in this direction establishing some remarkable results. A very important object used to do these kinds of computations is the classical stable homotopy category SHC. This category was first defined in [27] by Kan. Boardman, in his thesis [6], constructed the (derived) smash product on $S H C$ whose monoids represent multiplicative cohomology theories. In [8], Bousfield and Friedlander introduced a stable model category Sp of spectra with $\mathrm{Ho}(\mathrm{Sp})$ triangulated equivalent to $S H C$. The category Sp enjoys several nice point-set level properties. However, it does not possess a symmetric monoidal product that descends to Boardman's smash product on SHC. This initiated the search for new models for SHC that possess symmetric monoidal products. In the 1990s, several such models appeared: $S$-modules of Elmendorf, Kriz, Mandell and May [15], symmetric spectra from Hovey, Shipley and Smith [24], simplicial (continuous) functors from Lydakis [31], and orthogonal spectra from Mandell, May, Schwede and Shipley [34]. All these models turned out to be Quillen equivalent to Sp (and hence, to each other) and this naturally motivated the following:

Question How many models does $S H C$ admit up to Quillen equivalence? 
In [43], Schwede answered this question. He proved that the stable homotopy category is rigid, ie if $\mathscr{C}$ is a stable model category with $\operatorname{Ho}(\mathscr{C})$ triangulated equivalent to $S H C$, then the model categories $\mathscr{C}$ and Sp are Quillen equivalent. In other words, up to Quillen equivalence, there is a unique stable model category whose homotopy category is triangulated equivalent to the stable homotopy category. This result implies that all "higher order structure" of the stable homotopy theory, such as homotopy types of function spaces, is determined by the suspension functor and the class of homotopy cofiber sequences.

Generally, when passing from a model category $\mathscr{C}$ to its homotopy category $\mathrm{Ho}(\mathscr{C})$, one loses "higher homotopical information" such as homotopy types of mapping spaces in $\mathscr{C}$ or the algebraic $K$-theory of $\mathscr{C}$. In particular, the existence of a triangulated equivalence of homotopy categories does not necessarily imply that two given models are Quillen equivalent to each other. Here is an easy example of such a loss of information. Let Mod- $K(n)$ denote the model category of right modules over the $n^{\text {th }}$ Morava $K$-theory $K(n)$, and let $\mathbf{d g} \operatorname{Mod}-\pi_{*} K(n)$ denote the model category of differential graded modules over the graded homotopy ring $\pi_{*} K(n)$. Then the homotopy categories $\operatorname{Ho}(\operatorname{Mod}-K(n))$ and $\operatorname{Ho}\left(\mathbf{d g} \operatorname{Mod}-\pi_{*} K(n)\right)$ are triangulated equivalent, whereas the model categories Mod- $K(n)$ and $\mathbf{d g} \operatorname{Mod}-\pi_{*} K(n)$ are not Quillen equivalent. The reason is that the homotopy types of function spaces in $\mathbf{d g} \operatorname{Mod}-\pi_{*} K(n)$ are products of Eilenberg-MacLane spaces, which is not the case for Mod-K(n) (see eg [38, Proposition A.1.10]).

Another important example which we would like to recall is due to Schlichting. It is easy to see that, for any prime $p$, the homotopy categories $\operatorname{Ho}\left(\operatorname{Mod}-\mathbb{Z} / p^{2}\right)$ and $\operatorname{Ho}\left(\operatorname{Mod}-\mathbb{F}_{p}[t] /\left(t^{2}\right)\right)$ are triangulated equivalent. In [41], Schlichting shows that the algebraic $K$-theories of the subcategories of compact objects of $\operatorname{Mod}-\mathbb{Z} / p^{2}$ and $\operatorname{Mod}-\mathbb{F}_{p}[t] /\left(t^{2}\right)$ are different for $p \geq 5$. It then follows from Dugger and Shipley [11, Corollary 3.10] that the model categories $\operatorname{Mod}-\mathbb{Z} / p^{2}$ and $\operatorname{Mod}-\mathbb{F}_{p}[t] /\left(t^{2}\right)$ are not Quillen equivalent. Note that there is also a reinterpretation of this example in terms of differential graded algebras by Dugger and Shipley [12].

Initiated by Schwede's result, much research has been done in recent years on establishing essential uniqueness of models for certain homotopy categories. In [40], Roitzheim shows that the $K_{(2)}$-local stable homotopy category has a unique model. For other theorems of this type, see Barnes and Roitzheim [3], and Hutschenreuter [25].

The present work establishes a new uniqueness result. We prove an equivariant version of Schwede's rigidity theorem at the prime 2. Before formulating our main result, we would like to say a few words on equivariant stable homotopy theory. 
The $G$-equivariant stable homotopy category (indexed on a complete $G$-universe), for any compact Lie group $G$, was introduced in the book [30] by Lewis, May, Steinberger, and McClure. Roughly speaking, the objects of this category are $G$-spectra indexed on finite-dimensional $G$-representations. In this paper, we will work with the stable model category $\operatorname{Sp}_{G}^{O}$ of $G$-equivariant orthogonal spectra indexed on a complete $G$-universe; see Mandell and May [33]. The homotopy category of $\operatorname{Sp}_{G}^{O}$ is the $G$-equivariant stable homotopy category. The advantage of this model is that it possesses a symmetric monoidal product compatible with the model structure. As in the nonequivariant case, the $G$-equivariant stable homotopy category has some other monoidal models, such as the category of orthogonal $G$-spectra equipped with the $\mathbb{S}$-model structure (flat model structure) in Stolz's [48, Theorem 2.3.27], the model category of $S_{G}$-modules [33, Section IV.2] and the model category of $G$-equivariant continuous functors of Blumberg [5]. For a finite group $G$, the model categories of $G$-equivariant topological symmetric spectra in the sense of Mandell [32] and Hausmann [20] are also monoidal models for the $G$-equivariant stable homotopy category. Note that all these model categories are known to be $G-\mathbf{T o p}_{*}$-Quillen equivalent to each other; see [33, Theorem IV.1.1; 5, Theorem 1.3; 48, Proposition 2.3.31; 32; 20].

Now we return to the actual content of this paper. Suppose $G$ is a finite group and $H$ a subgroup of $G$. For any $g \in G$, let ${ }^{g} H$ denote the conjugate subgroup $g H^{-1}$. Then the map

$$
g: \Sigma_{+}^{\infty} G /{ }^{g} H \longrightarrow \Sigma_{+}^{\infty} G / H
$$

in the homotopy category $\operatorname{Ho}\left(\operatorname{Sp}_{G}^{O}\right)$, given by $[x] \mapsto[x g]$ on the point-set level, is called the conjugation map associated to $g$ and $H$. Further, if $K$ is another subgroup of $G$ such that $K \leq H$, then we have the restriction map

$$
\operatorname{res}_{K}^{H}: \Sigma_{+}^{\infty} G / K \longrightarrow \Sigma_{+}^{\infty} G / H,
$$

which is just the obvious projection on the point-set level. Moreover, there is also a map backwards, called the transfer map

$$
\operatorname{tr}_{K}^{H}: \Sigma_{+}^{\infty} G / H \longrightarrow \Sigma_{+}^{\infty} G / K,
$$

given by the Pontryagin-Thom construction; see eg [30, Section IV.3] or tom Dieck [10, Section II.8]. These morphisms generate the stable Burnside (orbit) category, which is the full preadditive subcategory of $\operatorname{Ho}\left(\operatorname{Sp}_{G}^{O}\right)$ with objects the stable orbits $\Sigma_{+}^{\infty} G / H$ for $H \leq G$; see [30, Section V.9] and also Lewis [29].

Let $G$ be a finite group. We say that a model category $\mathscr{C}$ is a $G$-equivariant stable model category if it is enriched, tensored and cotensored over the category $G-\mathbf{T o p}_{*}$ of pointed $G$-spaces in a compatible way (ie the pushout-product axiom holds) and if 
the adjunction

$$
S^{V} \wedge-: \mathscr{C} \rightleftarrows \mathscr{C}: \Omega^{V}(-)
$$

is a Quillen equivalence for any finite-dimensional orthogonal $G$-representation $V$.

All the models for the $G$-equivariant stable homotopy category mentioned above are $G$-equivariant stable model categories. Different kinds of equivariant spectra indexed on incomplete universes provide examples of $G-\mathbf{T o p}_{*}$-model categories that are not $G$-equivariant stable model categories, but are stable as underlying model categories.

Here is the main result of this paper.

Theorem 1.1.1 Let $G$ be a finite group, let $\mathscr{C}$ be a cofibrantly generated, proper, $G$-equivariant stable model category, and let $\mathrm{Sp}_{G}^{O}$,(2) denote the 2-localization of $\mathrm{Sp}_{G}^{O}$. Suppose that

$$
\Psi: \operatorname{Ho}\left(\operatorname{Sp}_{G}^{O},(2)\right) \stackrel{\sim}{\longrightarrow} \operatorname{Ho}(\mathscr{C})
$$

is an equivalence of triangulated categories such that

$$
\Psi\left(\Sigma_{+}^{\infty} G / H\right) \cong G / H_{+} \wedge^{\mathbf{L}} \Psi(\mathbb{S})
$$

for any $H \leq G$. Suppose further that the latter isomorphisms are natural with respect to the restrictions, conjugations and transfers. Then there is a zigzag of $G-\mathbf{T o p}_{*}-$ Quillen equivalences between $\mathscr{C}$ and $\operatorname{Sp}_{G}^{O}$,(2) .

In fact, we strongly believe that the following integral version of Theorem 1.1.1 should be true:

Conjecture 1.1.2 Let $G$ be a finite group and let $\mathscr{C}$ be a cofibrantly generated, proper, $G$-equivariant stable model category. Suppose that

$$
\Psi: \operatorname{Ho}\left(\operatorname{Sp}_{G}^{O}\right) \stackrel{\sim}{\longrightarrow} \operatorname{Ho}(\mathscr{C})
$$

is an equivalence of triangulated categories such that

$$
\Psi\left(\Sigma_{+}^{\infty} G / H\right) \cong G / H_{+} \wedge^{\mathbf{L}} \Psi(\mathbb{S})
$$

for any $H \leq G$. Suppose further that the latter isomorphisms are natural with respect to the restrictions, conjugations and transfers. Then there is a zigzag of $G-\mathbf{T o p}_{*}$-Quillen equivalences between $\mathscr{C}$ and $\operatorname{Sp}_{G}^{O}$.

Note that if $G$ is trivial, then the statement of Conjecture 1.1.2 is true. This is Schwede's rigidity theorem [43] (or, more precisely, a special case of it, as the model category in Schwede's theorem need not be cofibrantly generated, topological or proper). In particular, the solution of Conjecture 1.1.2 would imply that all "higher-order structure" 
of the $G$-equivariant stable homotopy theory, such as the equivariant homotopy types of function $G$-spaces, is determined by the suspension functor, the class of homotopy cofiber sequences and the basic $\pi_{0}$-information of $\mathrm{Ho}\left(\mathrm{Sp}_{G}^{O}\right)$, ie the stable Burnside (orbit) category.

The proof of Theorem 1.1.1 is divided into two main parts: the first is categorical and the second is computational. The categorical part of the proof is mainly discussed in Section 3 and would essentially reduce a proof of Conjecture 1.1.2 to showing that a certain exact endofunctor

$$
F: \operatorname{Ho}\left(\operatorname{Sp}_{G}^{O}\right) \longrightarrow \operatorname{Ho}\left(\operatorname{Sp}_{G}^{O}\right)
$$

is an equivalence of categories. The computational part shows that, 2-locally, the endofunctor is indeed an equivalence of categories. The proof starts by generalizing Schwede's arguments from [42] to free (naive) $G$-spectra. From this point on, classical techniques of equivariant stable homotopy theory enter the proof. These include the double coset formula, Wirthmüller isomorphism, geometric fixed points, isotropy separation and the tom Dieck splitting. The central idea is to do induction on the order of subgroups and use the case of free $G$-spectra as the induction basis.

The only part of the proof of Theorem 1.1.1 which uses that we are working 2-locally is the part about free $G$-spectra in Section 4. The essential fact one needs here is that the self map $2 \cdot \mathrm{id}: M(2) \rightarrow M(2)$ of the $\bmod 2$ Moore spectrum is not zero in the stable homotopy category. For $p$ an odd prime, the map $p \cdot \mathrm{id}: M(p) \rightarrow M(p)$ is equal to zero, and this makes a big difference between the 2-primary and odd primary cases. Observe that the nontriviality of $2 \cdot \mathrm{id}: M(2) \longrightarrow M(2)$ amounts to the fact that $M(2)$ does not possess an $A_{2}$-structure with respect to the canonical unit map $\mathbb{S} \rightarrow M(2)$. In fact, for any prime $p$, the mod $p$ Moore spectrum $M(p)$ has an $A_{p-1}$-structure but does not admit an $A_{p}$-structure; see Angeltveit [2]. The obstruction for the latter is the element $\alpha_{1} \in \pi_{2 p-3} \mathbb{S}_{(p)}$. This is used by Schwede to obtain the integral rigidity result for the stable homotopy category in [43]. It seems to be rather nontrivial to generalize Schwede's obstruction theory arguments about coherent actions of Moore spaces [43] to the equivariant case.

This paper is organized as follows. Section 2 contains some basic facts about model categories, triangulated categories and $G$-equivariant orthogonal spectra. We also review the level and stable model structures on the category of orthogonal $G$-spectra. In Section 3, we discuss the categorical part of the proof. Here we introduce the category of orthogonal $G$-spectra $\operatorname{Sp}_{G}^{O}(\mathscr{C})$ internal to an equivariant model category $\mathscr{C}$, and we show that if $\mathscr{C}$ is stable in an equivariant sense and additionally satisfies certain technical conditions, then $\mathscr{C}$ and $\operatorname{Sp}_{G}^{O}(\mathscr{C})$ are Quillen equivalent. This allows us to reduce the proof of Theorem 1.1.1 to showing that a certain exact endofunctor $F$ 
of $\operatorname{Ho}\left(\operatorname{Sp}_{G}^{O}\right.$, (2) $)$ is an equivalence of categories. In Section 4, we show that $F$ becomes an equivalence when restricted to the full subcategory of free $G$-spectra.

In Section 5, we prove a technical result that reduces the proof of the main theorem to checking that the map

$$
F:\left[\Sigma_{+}^{\infty} G / H, \Sigma_{+}^{\infty} G / H\right]_{*}^{G} \longrightarrow\left[F\left(\Sigma_{+}^{\infty} G / H\right), F\left(\Sigma_{+}^{\infty} G / H\right)\right]_{*}^{G}
$$

is an isomorphism for any subgroup $H$ of $G$. This is then verified inductively in Section 7. The results of Section 4 are used for the induction basis. The induction step uses geometric fixed points and a certain short exact sequence that we discuss in Section 6.

\section{Preliminaries}

\subsection{Model categories}

A model category is defined as a bicomplete category $\mathscr{C}$ equipped with three classes of morphisms, called weak equivalences, fibrations and cofibrations, satisfying certain axioms. We will not list these axioms here. The point of this structure is that it allows one to "do homotopy theory" in $\mathscr{C}$. Good references for model categories include [13], [23] and [39].

The fundamental example of a model category is the category of topological spaces; see [39; 23, Theorem 2.4.19]. Further important examples are the category of simplicial sets (see [39; 17, Theorem I.11.3]) and the category of chain complexes of modules over a ring as in [23, Theorem 2.3.11].

For any model category $\mathscr{C}$, one has the associated homotopy category $\operatorname{Ho}(\mathscr{C})$, which is defined as the localization of $\mathscr{C}$ with respect to the class of weak equivalences; see eg [23, Section 1.2] or [13]. The model structure guarantees that we do not face set-theoretic problems when passing to localization, ie $\mathrm{Ho}(\mathscr{C})$ has Hom-sets.

A Quillen adjunction between two model categories $\mathscr{C}$ and $\mathscr{D}$ is defined to be a pair of adjoint functors

$$
F: \mathscr{C} \rightleftarrows \mathscr{D}: E
$$

where the left adjoint $F$ preserves cofibrations and acyclic cofibrations (or, equivalently, $E$ preserves fibrations and acyclic fibrations). We refer to $F$ as a left Quillen functor and to $E$ as a right Quillen functor. Quillen's total derived functor theorem (see eg [39] or [17, Theorem II.8.7]) says that any such pair of adjoint functors induces an adjunction

$$
\mathbf{L} F: \operatorname{Ho}(\mathscr{C}) \rightleftarrows \operatorname{Ho}(\mathscr{D}): \mathbf{R} E
$$


The functor $\mathbf{L} F$ is called the left derived functor of $F$ and $\mathbf{R} E$ the right derived functor of $E$. If $\mathbf{L} F$ is an equivalence of categories (or, equivalently, $\mathbf{R} E$ is an equivalence), then the Quillen adjunction is called a Quillen equivalence.

Next, let us quickly review cofibrantly generated model categories. Here we mainly follow [23, Section 2.1]. Let $I$ be a set of morphisms in an arbitrary cocomplete category. A relative I-cell complex is a morphism that is a (possibly transfinite) composition of pushouts of coproducts of maps in I. A map is called I-injective if it has the right lifting property with respect to $I$. An I-cofibration is map that has the left lifting property with respect to $I$-injective maps. The class of $I$-cell complexes will be denoted by $I$-cell. Next, $I$-inj will stand for the class of $I$-injective maps and $I$-cof for the class of $I$-cofibrations. It is easy to see that $I$-cell $\subset I$-cof. Finally, let us recall the notion of smallness. An object $K$ of a cocomplete category is small with respect to a given class $\mathcal{I}$ of morphisms if the representable functor associated to $K$ commutes with colimits of large enough transfinite sequences of morphisms from $\mathcal{I}$. See [23, Definition 2.13] for more details.

Definition 2.1.1 [23, Definition 2.1.17] Let $\mathscr{C}$ be a model category. We say that $\mathscr{C}$ is cofibrantly generated if there are sets $I$ and $J$ of maps in $\mathscr{C}$ such that:

(i) The domains of $I$ and $J$ are small relative to $I$-cell and $J$-cell, respectively.

(ii) The class of fibrations is $J$-inj.

(iii) The class of acyclic fibrations is $I-$ inj.

Here is a general result that will be used in this paper.

Proposition 2.1.2 (See eg [23, Theorem 2.1.19]) Let $\mathscr{C}$ be a category with small limits and colimits. Suppose that $\mathscr{W}$ is a subcategory of $\mathscr{C}$ and that $I$ and $J$ are sets of morphisms of $\mathscr{C}$. Assume that the following conditions are satisfied:

(i) The subcategory $\mathscr{W}$ satisfies the so-called "two out of three" property and is closed under retracts.

(ii) The domains of $I$ and $J$ are small relative to $I$-cell and $J$-cell, respectively.

(iii) $J$-cell $\subset \mathscr{W} \cap I$-cof.

(iv) $\quad I-i n j=\mathscr{W} \cap J-i n j$.

Then $\mathscr{C}$ is a cofibrantly generated model category with $\mathscr{W}$ the class of weak equivalences, $J$-inj the class fibrations and I-cof the class of cofibrations. 
Note that the set $I$ is usually referred to as a set of generating cofibrations and $J$ as a set of generating acyclic cofibrations.

Furthermore, we recall the definitions of monoidal model categories and enriched model categories.

Definition 2.1.3 (See eg [23, Definition 4.2.6]) A monoidal model category is a closed symmetric monoidal category $\mathscr{V}$ together with a model structure such that the following conditions hold:

(i) (The pushout-product axiom) Let $i: K \rightarrow L$ and $j: A \rightarrow B$ be cofibrations in the model category $\mathscr{V}$. Then the induced map

$$
i \square j: K \wedge B \vee_{K \wedge A} L \wedge A \longrightarrow L \wedge B
$$

is a cofibration in $\mathscr{V}$. Furthermore, if either $i$ or $j$ is an acyclic cofibration, then so is $i \square j$.

(ii) Let $q: Q I \rightarrow I$ be a cofibrant replacement for the unit $I$. Then the maps

$$
q \wedge 1: Q I \wedge X \longrightarrow I \wedge X \text { and } 1 \wedge q: X \wedge Q I \longrightarrow X \wedge I
$$

are weak equivalences for any cofibrant $X$.

Definition 2.1.4 (See eg [23, Definition 4.2.18]) Let $\mathscr{V}$ be a monoidal model category. A $\mathscr{V}$-model category is a model category $\mathscr{C}$ with the following data and properties:

(i) The category $\mathscr{C}$ is enriched, tensored and cotensored over $\mathscr{V}$; see [28, Sections 1.2, 3.7]. This means that we have tensors $K \wedge X$, cotensors $X^{K}$ and mapping objects $\operatorname{Hom}(X, Y) \in \mathscr{V}$ for $K \in \mathscr{V}$ and $X, Y \in \mathscr{C}$, and that all these functors are related by $\mathscr{V}$-enriched adjunctions

$$
\operatorname{Hom}(K \wedge X, Y) \cong \operatorname{Hom}\left(X, Y^{K}\right) \cong \operatorname{Hom}(K, \operatorname{Hom}(X, Y)) \text {. }
$$

(ii) (The pushout-product axiom) Let $i: K \rightarrow L$ be a cofibration in the model category $\mathscr{V}$ and $j: A \rightarrow B$ a cofibration in the model category $\mathscr{C}$. Then the induced map

$$
i \square j: K \wedge B \vee_{K \wedge A} L \wedge A \longrightarrow L \wedge B
$$

is a cofibration in $\mathscr{C}$. Furthermore, if either $i$ or $j$ is an acyclic cofibration, then so is $i \square j$.

(iii) If $q: Q I \rightarrow I$ is a cofibrant replacement for the unit $I$ in $\mathscr{V}$, then the induced map $q \wedge 1: Q I \wedge X \rightarrow I \wedge X$ is a weak equivalence in $\mathscr{C}$ for any cofibrant $X$. 
Finally, let us recall the definition of a proper model category.

Definition 2.1.5 A model category is called left proper if weak equivalences are preserved by pushouts along cofibrations. Dually, a model category is called right proper if weak equivalences are preserved by pullbacks along fibrations. A model category which is left proper and right proper is said to be proper.

\subsection{Triangulated categories and stable model categories}

We start this subsection by recalling some basics about triangulated categories. The references for triangulated categories include [16, Chapter IV; 37; 50, Section 10.2].

A triangulated category is an additive category $\mathcal{T}$ together with a self equivalence $\Sigma: \mathcal{T} \longrightarrow \mathcal{T}$ (suspension functor) and a class of triangles in $\mathcal{T}$ of type

$$
X \stackrel{f}{\longrightarrow} Y \stackrel{g}{\longrightarrow} Z \stackrel{h}{\longrightarrow} \Sigma X,
$$

called distinguished triangles. These are subject to certain conditions which axiomatize the mapping cone sequences from homological algebra or cofiber sequences from algebraic topology. We do not recall these conditions here and refer to [16, Chapter IV; 37; 50, Section 10.2] for details.

An exact (or triangulated) functor between triangulated categories $\mathcal{T}$ and $\mathcal{S}$ is an additive functor $F: \mathcal{T} \rightarrow \mathcal{S}$ together with a natural isomorphism $\alpha: F \circ \Sigma \cong \Sigma \circ F$ such that, for any distinguished triangle

$$
X \stackrel{f}{\longrightarrow} Y \stackrel{g}{\longrightarrow} Z \stackrel{h}{\longrightarrow} \Sigma X
$$

in $\mathcal{T}$, the triangle

$$
F(X) \stackrel{F(f)}{\longrightarrow} F(Y) \stackrel{F(g)}{\longrightarrow} F(Z) \stackrel{\alpha \circ F(h)}{\longrightarrow} \Sigma F(X)
$$

is distinguished in $\mathcal{S}$.

A natural transformation $\theta: F \longrightarrow G$ of exact functors is a said to be triangulated if it commutes with the suspension isomorphisms, ie if the following diagram commutes:

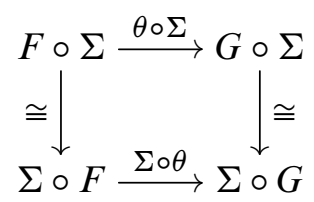

Let $\mathcal{T}$ be a triangulated category with infinite coproducts. A full triangulated subcategory $\mathcal{S} \subset \mathcal{T}$ is called localizing if it is closed under infinite coproducts. A set of 
objects $\mathcal{P}$ in $\mathcal{T}$ is said to generate the triangulated category $\mathcal{T}$ if the smallest localizing subcategory of $\mathcal{T}$ containing $\mathcal{P}$ coincides with $\mathcal{T}$.

Suppose now that we are given a triangulated category $\mathcal{T}$ with infinite coproducts and a set of objects $\mathcal{P}$ in $\mathcal{T}$ consisting of compact objects (an object $P$ is called compact if $\mathcal{T}(P,-)$ commutes with infinite coproducts). Then by [47, Lemma 2.2.1], the following are equivalent:

(i) The set $\mathcal{P}$ generates $\mathcal{T}$.

(ii) An object $X$ of $\mathcal{T}$ is trivial if and only if $\mathcal{T}\left(\Sigma^{n} P, X\right)=0$ for any $P \in \mathcal{P}$ and integer $n$.

The following two lemmas will be very useful.

Lemma 2.2.1 Let $\mathcal{T}$ and $\mathcal{S}$ be triangulated categories with infinite coproducts and a set $\mathcal{P}$ of generators for $\mathcal{T}$. Further, suppose $F, G: \mathcal{T} \longrightarrow \mathcal{S}$ are exact functors which commute with infinite coproducts, and $\theta: F \longrightarrow G$ is a triangulated natural transformation. If $\theta(P): F(P) \rightarrow G(P)$ is an isomorphism for any $P \in \mathcal{P}$, then $\theta$ is a natural isomorphism of functors.

Proof Consider the full subcategory of $\mathcal{T}$ consisting of those objects $X$ for which

$$
\theta(X): F(X) \longrightarrow G(X)
$$

is an isomorphism. This full subcategory is a triangulated subcategory and is closed under infinite coproducts, ie it is a localizing subcategory. By our assumption, this localizing subcategory contains the set $\mathcal{P}$. Since $\mathcal{P}$ generates $\mathcal{T}$, the minimal localizing subcategory containing $\mathcal{P}$ coincides with $\mathcal{T}$. Hence $\theta(X): F(X) \rightarrow G(X)$ is an isomorphism for any $X$ in $\mathcal{T}$.

Before stating the second lemma, we introduce the notation $\mathcal{T}(X, Y)_{*}$ for $\mathcal{T}\left(\Sigma^{*} X, Y\right)$.

Lemma 2.2.2 Let $\mathcal{T}$ be a triangulated category with infinite coproducts and with a set of compact generators $\mathcal{P}$. Further, suppose $F: \mathcal{T} \longrightarrow \mathcal{T}$ is an exact functor that commutes with infinite coproducts and, for any $P \in \mathcal{P}$, the object $F(P)$ is isomorphic to $P$. If the map

$$
F: \mathcal{T}\left(P, P^{\prime}\right)_{*} \longrightarrow \mathcal{T}\left(F(P), F\left(P^{\prime}\right)\right)_{*}
$$

is an isomorphism for any $P, P^{\prime} \in \mathcal{P}$, then $F$ is an equivalence of categories (here we implicitly identify $F\left(\Sigma^{*} P\right)$ with $\left.\Sigma^{*} F(P)\right)$. 
Proof For any fixed $P \in \mathcal{P}$, the full subcategory of those objects $Y$ in $\mathcal{T}$ for which

$$
F: \mathcal{T}(P, Y)_{*} \longrightarrow \mathcal{T}(F(P), F(Y))_{*}
$$

is an isomorphism is a localizing subcategory of $\mathcal{T}$. By our assumptions, $\mathcal{P}$ is contained in this localizing subcategory, and $\mathcal{P}$ generates $\mathcal{T}$. Hence the latter localizing subcategory coincides with $\mathcal{T}$. Now we want to show that

$$
F: \mathcal{T}(X, Y)_{*} \longrightarrow \mathcal{T}(F(X), F(Y))_{*}
$$

is an isomorphism for any $X$ and $Y$. For any fixed $Y$, consider the full subcategory of those objects $X$ of $\mathcal{T}$ for which the latter morphism is an isomorphism. Again we see that this subcategory is localizing, and by the previous paragraph, it contains $\mathcal{P}$. Thus it coincides with $\mathcal{T}$, and we conclude that $F$ is fully faithful.

Finally, since $F$ is fully faithful, exact and commutes with infinite coproducts, its essential image is a localizing subcategory. By assumption, $\mathcal{P}$ is contained in the essential image of $F$. Hence the essential image of $F$ coincides with $\mathcal{T}$.

The examples of triangulated categories we are interested in come from stable model categories. Recall $[39 ; 23$, Definition 6.1 .1$]$ that the homotopy category $\operatorname{Ho}(\mathscr{C})$ of a pointed model category $\mathscr{C}$ supports a suspension functor

$$
\Sigma: \operatorname{Ho}(\mathscr{C}) \longrightarrow \operatorname{Ho}(\mathscr{C})
$$

with a right adjoint loop functor

$$
\Omega: \operatorname{Ho}(\mathscr{C}) \longrightarrow \operatorname{Ho}(\mathscr{C}) .
$$

If the functors $\Sigma$ and $\Omega$ are inverse equivalences, then the pointed model category $\mathscr{C}$ is called a stable model category. For any stable model category $\mathscr{C}$, the homotopy category $\operatorname{Ho}(\mathscr{C})$ is naturally triangulated; see [23, Section 7.1] and also [39, Section I.4]. The suspension functor is the shift, and the distinguished triangles come from the cofiber sequences.

Examples of stable model categories are the model category of chain complexes and also various model categories of spectra ( $S$-modules [15], orthogonal spectra [34], symmetric spectra [24], sequential spectra [8]).

Next, suppose

$$
F: \mathscr{C} \rightleftarrows \mathscr{D}: E
$$

is a Quillen adjunction between stable model categories $\mathscr{C}$ and $\mathscr{D}$. Then by [23, Section 7.1] (see also [39, Section I.4]), the derived functors

$$
\mathbf{L} F: \operatorname{Ho}(\mathscr{C}) \rightleftarrows \operatorname{Ho}(\mathscr{D}): \mathbf{R} E \text {. }
$$


are exact functors of triangulated categories, and the derived unit id $\rightarrow \mathbf{R} E \circ \mathbf{L} F$ and counit $\mathbf{L} F \circ \mathbf{R} E \rightarrow$ id are triangulated natural transformations.

For any stable model category $\mathscr{C}$ and objects $X, Y \in \mathscr{C}$, we will denote the abelian group of morphisms from $X$ to $Y$ in $\operatorname{Ho}(\mathscr{C})$ by $[X, Y]^{\mathrm{Ho}(\mathscr{C})}$.

\section{3 $G$-equivariant spaces}

Convention 2.3.1 In this paper, $G$ will always denote a finite group.

Convention 2.3.2 By a topological space, we will always mean a compactly generated weak Hausdorff space.

The category $G$-Top $*$ of pointed topological $G$-spaces admits a proper and cofibrantly generated model structure such that $f: X \rightarrow Y$ is a weak equivalence (resp. fibration) if the induced map on $H$-fixed points

$$
f^{H}: X^{H} \longrightarrow Y^{H}
$$

is a weak homotopy equivalence (resp. Serre fibration) for any subgroup $H \leq G$; see eg [33, Section III.1]. The set of $G$-maps

$$
\left(G / H \times S^{n-1}\right)_{+} \longrightarrow\left(G / H \times D^{n}\right)_{+}, \quad n \geq 0, H \leq G
$$

generates cofibrations in this model structure. The acyclic cofibrations are generated by the maps

$$
\text { incl }_{0}:\left(G / H \times D^{n}\right)_{+} \longrightarrow\left(G / H \times D^{n} \times I\right)_{+}, \quad n \geq 0, H \leq G .
$$

The model category $G-$ Top $_{*}$ is a closed symmetric monoidal model category; see [33, Section III.1]. The monoidal product on $G-\mathbf{T o p}_{*}$ is given by the smash product $X \wedge Y$, with the diagonal $G$-action, for any $X, Y \in G$-Top , $_{*}$ and the mapping object is the nonequivariant pointed mapping space $\operatorname{Map}(X, Y)$ with the conjugation $G$-action.

\section{$2.4 G$-equivariant orthogonal spectra}

We start by reminding the reader about the definition of an orthogonal spectrum [34].

Definition 2.4.1 An orthogonal spectrum $X$ consists of the following data:

- A sequence of pointed spaces $X_{n}$, for $n \geq 0$.

- A base-point preserving continuous action of the orthogonal group $O(n)$ on $X_{n}$ for each $n \geq 0$.

- Continuous based maps $\sigma_{n}: X_{n} \wedge S^{1} \longrightarrow X_{n+1}$. 
This data is subject to the following condition: for all $n, m \geq 0$, the iterated structure map $X_{n} \wedge S^{m} \rightarrow X_{n+m}$ is $O(n) \times O(m)$-equivariant.

Next, let us recall the definition of $G$-equivariant orthogonal spectra. Here we mainly follow [45]; see also [33], which is the original source for $G$-equivariant orthogonal spectra.

Definition 2.4.2 An orthogonal $G$-spectrum ( $G$-equivariant orthogonal spectrum) is an orthogonal spectrum $X$ equipped with a categorical $G$-action, ie with a group homomorphism $G \rightarrow \operatorname{Aut}(X)$.

The category of orthogonal $G$-spectra is denoted by $\operatorname{Sp}_{G}^{O}$. Any orthogonal $G$-spectrum $X$ can be evaluated on an arbitrary finite-dimensional orthogonal $G$-representation $V$. The $G$-space $X(V)$ is defined by

$$
X(V)=\mathbf{L}\left(\mathbb{R}^{n}, V\right)_{+} \wedge O(n) X_{n},
$$

where the number $n$ is the dimension of $V$, the vector space $\mathbb{R}^{n}$ is equipped with the standard scalar product and $\mathbf{L}\left(\mathbb{R}^{n}, V\right)$ is the space of (not necessarily equivariant) linear isometries from $\mathbb{R}^{n}$ to $V$. The $G$-action on $X(V)$ is given diagonally:

$$
g \cdot[\varphi, x]=[g \varphi, g x], \quad g \in G, \varphi \in \mathbf{L}\left(\mathbb{R}^{n}, V\right), x \in X_{n} .
$$

For the trivial $G$-representation $\mathbb{R}^{n}$, the pointed $G$-space $X\left(\mathbb{R}^{n}\right)$ is canonically isomorphic to the pointed $G$-space $X_{n}$. Next, let $S^{V}$ denote the representation sphere of $V$, ie the one-point compactification of $V$. Using the iterated structure maps of $X$, for any finite-dimensional orthogonal $G$-representations $V$ and $W$, one can define $G$-equivariant generalized structure maps

$$
\sigma_{V, W}: X(V) \wedge S^{W} \longrightarrow X(V \oplus W) .
$$

These are then used to define $G$-equivariant homotopy groups

$$
\pi_{k}^{G} X=\operatorname{colim}_{n}\left[S^{k+n \rho_{G}}, X\left(n \rho_{G}\right)\right]^{G}, \quad k \in \mathbb{Z},
$$

where $\rho_{G}$ denotes the regular representation of $G$. Furthermore, for any subgroup $H \leq G$ and integer $k$, one defines $\pi_{k}^{H} X$ to be the $k^{\text {th }} H$-equivariant homotopy group of $X$ considered as an $H$-spectrum.

Definition 2.4.3 A map $f: X \rightarrow Y$ of $G$-equivariant orthogonal spectra is called a stable equivalence if the induced map

$$
\pi_{k}^{H}(f): \pi_{k}^{H} X \longrightarrow \pi_{k}^{H} Y
$$

is an isomorphism for any integer $k$ and any subgroup $H \leq G$. 


\subsection{Comparison of different definitions}

Before continuing the recollection, let us explain the relation of Definition 2.4.2 with the original definition of $G$-equivariant orthogonal spectra due to Mandell and May. For this, we first recall the category $O_{G}$. The objects of $O_{G}$ are finite-dimensional orthogonal $G$-representations. For any orthogonal $G$-representations $V$ and $W$, the pointed morphism $G$-space $O_{G}(V, W)$ is defined to be the Thom space of the $G$-equivariant vector bundle

$$
\xi(V, W) \longrightarrow \mathbf{L}(V, W),
$$

where $\mathbf{L}(V, W)$ is the space of linear isometric embeddings from $V$ to $W$, and

$$
\xi(V, W)=\{(f, x) \in \mathbf{L}(V, W) \times W \mid x \perp f(V)\} .
$$

For more details about this category, see [33, Section II.4]. It follows from [33, Theorem V.1.5] (see also [21, Proposition A.19]) that the category of $O_{G}$-spaces (which is the category of $G-\mathbf{T o p}_{*}$-enriched functors from $O_{G}$ to $G-\mathbf{T o p}_{*}$ ) is equivalent to the category of $G$-equivariant orthogonal spectra.

Remark 2.5.1 In [33, Section II.2], Mandell and May define $G$-equivariant orthogonal spectra indexed on a $G$-universe $\mathscr{U}$ (which is a countably infinite-dimensional real inner product space with certain properties; see [33, Definition II.1.1]). Such a $G-$ spectrum is a collection of $G$-spaces indexed on those representations that embed into $\mathscr{U}$ together with certain equivariant structure maps. It follows from [33, Theorems II.4.3, V.1.5] that for any $G$-universe $\mathscr{U}$, the category of orthogonal $G$-spectra indexed on $\mathscr{U}$ and the category $\operatorname{Sp}_{G}^{O}$ are equivalent. This shows that universes are not relevant for the point-set level definition of an orthogonal $G$-spectrum. However, they become important when one considers the homotopy theory of orthogonal $G$-spectra. We will use the homotopy theory of orthogonal $G$-spectra where all finite-dimensional orthogonal $G$-representations are built in. This means that we will work with the genuine $G$-spectra or, in other words, with the homotopy theory of orthogonal $G-$ spectra indexed on a complete universe (as a model of such a universe one can take the sum $\infty \rho_{G}$ of countable copies of the regular representation $\rho_{G}$ ).

Next, the category $\operatorname{Sp}_{G}^{O}$ is a closed symmetric monoidal category. The symmetric monoidal structure on $\mathrm{Sp}_{G}^{O}$ is given by the smash product of underlying orthogonal spectra (see [34]) with the diagonal $G$-action. Further, for any universe $\mathscr{U}$, the category of $G$-equivariant orthogonal spectra indexed on $\mathscr{U}$, as well as the category of $O_{G}-$ spaces, are closed symmetric monoidal categories. It follows from [33, Theorems II.4.3, V.1.5] (see also [21, Proposition A.19]) that all the equivalences discussed above are, in fact, equivalences of closed symmetric monoidal categories. 
From this point on we will freely use all the results of [33] for the category $\operatorname{Sp}_{G}^{O}$ having the above equivalences in mind.

\subsection{The level model structure on $\mathrm{Sp}_{G}^{O}$}

In this subsection we closely follow [33, Section III.2].

For any finite group $G$, the category $O_{G}$ is skeletally small, ie there is a small full subcategory sk $O_{G}$ of $O_{G}$ such that the embedding sk $O_{G} \hookrightarrow O_{G}$ is an equivalence of categories. Such a subcategory is called a small skeleton of $O_{G}$. We can fix once and for all a small skeleton sk $O_{G}$ of $O_{G}$.

Next, for any finite-dimensional orthogonal $G$-representation $V$, the evaluation functor

$$
\operatorname{Ev}_{V}: \operatorname{Sp}_{G}^{O} \longrightarrow G-\mathbf{T o p}_{*}
$$

given by $X \mapsto X(V)$, has a left adjoint $G-\mathbf{T o p}_{*}$-functor

$$
F_{V}: G-\mathbf{T o p}_{*} \longrightarrow \mathrm{Sp}_{G}^{O}
$$

which is defined by (see [33, Section II.4])

$$
F_{V} A(W)=O_{G}(V, W) \wedge A .
$$

Let $I_{1 \mathrm{v}}^{G}$ denote the set of morphisms

$$
\left.\left\{F_{V}\left(G / H \times S^{n-1}\right)_{+}\right) \longrightarrow F_{V}\left(\left(G / H \times D^{n}\right)_{+}\right) \mid V \in \operatorname{sk} O_{G}, n \geq 0, H \leq G\right\}
$$

and $J_{\mathrm{lv}}^{G}$ denote the set of morphisms

$$
\left\{F_{V}\left(\left(G / H \times D^{n}\right)_{+}\right) \longrightarrow F_{V}\left(\left(G / H \times D^{n} \times I\right)_{+}\right) \mid V \in \operatorname{sk} O_{G}, n \geq 0, H \leq G\right\} .
$$

In other words, the sets $I_{1 \mathrm{v}}^{G}$ and $J_{1 \mathrm{v}}^{G}$ are obtained by applying the functors $F_{V}$, for $V \in$ sk $O_{G}$, to the generating cofibrations and generating acyclic cofibrations of $G-\mathbf{T o p}_{*}$, respectively. Further, we recall

Definition 2.6.1 Let $f: X \rightarrow Y$ be a morphism in $\operatorname{Sp}_{G}^{O}$. The map $f$ is called a level equivalence if $f(V): X(V) \rightarrow Y(V)$ is a weak equivalence in $G$-Top $*$ for any $V \in \operatorname{sk} O_{G}$. It is called a level fibration if $f(V): X(V) \rightarrow Y(V)$ is a fibration in $G-$ Top $_{*}$ for any $V \in \operatorname{sk} O_{G}$. A map in $\operatorname{Sp}_{G}^{O}$ is called a cofibration if it has the left lifting property with respect to all maps that are level fibrations and level equivalences (ie level acyclic fibrations).

Proposition 2.6.2 [33, Theorem III.2.4] The category $\mathrm{Sp}_{G}^{O}$, together with level equivalences, level fibrations and cofibrations, forms a cofibrantly generated, proper model category. The set $I_{\mathrm{lv}}^{G}$ serves as a set of generating cofibrations and the set $J_{1 \mathrm{v}}^{G}$ serves as a set of generating acyclic cofibrations. 


\subsection{The stable model structure on $\mathrm{Sp}_{G}^{O}$}

The reference for this subsection is [33, Section III.4].

Recall that for any $G$-equivariant orthogonal spectrum $X$, we have the generalized structure maps

$$
\sigma_{V, W}: X(V) \wedge S^{W} \longrightarrow X(V \oplus W) .
$$

Let $\tilde{\sigma}_{V, W}: X(V) \rightarrow \Omega^{W} X(V \oplus W)$ denote the adjoint of $\sigma_{V, W}$.

Definition 2.7.1 An orthogonal $G$-spectrum $X$ is called a $G-\Omega$-spectrum if the maps $\widetilde{\sigma}_{V, W}$ are weak equivalences in $G-\mathbf{T o p}_{*}$ for any $V$ and $W$ in $O_{G}$.

Before formulating the theorem about the stable model structure on $\mathrm{Sp}_{G}^{O}$, let us recall certain morphisms in $\mathrm{Sp}_{G}^{O}$ that will form a generating set of acyclic cofibrations for this model structure. Let $V, W \in \operatorname{sk} O_{G}$ and

$$
\lambda_{V, W}: F_{V \oplus W} S^{W} \longrightarrow F_{V} S^{0}
$$

denote the map of $G$-equivariant orthogonal spectra that is adjoint to the map

$$
S^{W} \longrightarrow \operatorname{Ev}_{V \oplus W}\left(F_{V} S^{0}\right)=O_{G}(V, V \oplus W)
$$

that sends $z \in W$ to $(V \stackrel{(1,0)}{\longrightarrow} V \oplus W, z)$; see [33, Definition III.4.3]. Using the mapping cylinder construction, the map $\lambda_{V, W}$ factors as a composite

$$
F_{V \oplus W} S^{W} \stackrel{\kappa_{V, W}}{\longrightarrow} M \lambda_{V \oplus W} \stackrel{r_{V \oplus W}}{\longrightarrow} F_{V} S^{0},
$$

where $r_{V \oplus W}$ is a $G$-equivariant homotopy equivalence, and $\kappa_{V, W}$ is a cofibration and a stable equivalence [33, Lemma III.4.5, Definition III.4.6]. Now consider any generating cofibration

$$
i:\left(G / H \times S^{n-1}\right)_{+} \longrightarrow\left(G / H \times D^{n}\right)_{+} .
$$

Let $i \square \kappa V, W$ denote the pushout-product induced from this commutative square:

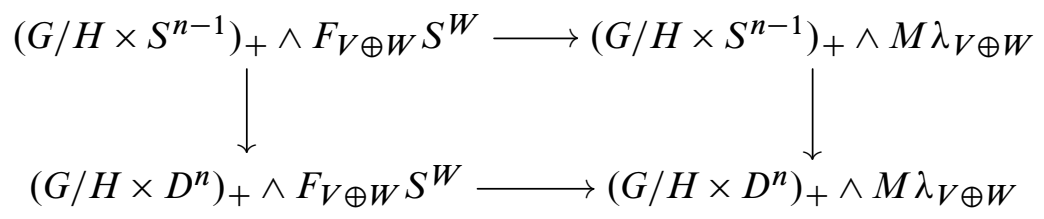

Define

$$
K^{G}=\left\{i \square \kappa_{V, W} \mid V, W \in \operatorname{sk} O_{G}, H \leq G, n \geq 0\right\} .
$$


Let $J_{\text {st }}^{G}$ stand for the union $J_{1 \mathrm{v}}^{G} \cup K^{G}$. For convenience, we will also introduce the notation $I_{\mathrm{st}}^{G}=I_{\mathrm{lv}}^{G}$.

Before stating the main theorem of this subsection, we need the following definition.

Definition 2.7.2 A map $f: X \rightarrow Y$ of orthogonal $G$-spectra is called a stable fibration, if it has the right lifting property with respect to the maps that are cofibrations and stable equivalences.

Theorem 2.7.3 [33, Theorem III.4.2] The category $\mathrm{Sp}_{G}^{O}$, together with cofibrations, stable equivalences and stable fibrations, forms a proper, cofibrantly generated, stable model category. The set $I_{\mathrm{st}}^{G}$ generates cofibrations and the set $J_{\text {st }}^{G}$ generates acyclic cofibrations. Furthermore, the fibrant objects are precisely the $G-\Omega$-spectra.

The category $\mathrm{Sp}_{G}^{O}$, together with the latter model structure, is referred to as the stable model category of orthogonal $G$-spectra (indexed on a complete $G$-universe). From now on, the notation $\mathrm{Sp}_{G}^{O}$ will always stand for this model category.

Finally, we recall that the stable model category $\mathrm{Sp}_{G}^{O}$, together with the smash product, forms a closed symmetric monoidal model category [33, Section III.7]. In particular, the following holds:

Proposition 2.7.4 Suppose that $i: K \rightarrow L$ and $j: A \rightarrow B$ are cofibrations in $\operatorname{Sp}_{G}^{O}$. Then the pushout-product

$$
i \square j: K \wedge B \vee_{K \wedge A} L \wedge A \longrightarrow L \wedge B
$$

is a cofibration in $\operatorname{Sp}_{G}^{O}$. The map $i \square j$ is also a stable equivalence if, in addition, $i$ or $j$ is a stable equivalence.

\subsection{The equivariant stable homotopy category}

In this subsection, we list some well known properties of the homotopy category $\operatorname{Ho}\left(\operatorname{Sp}_{G}^{O}\right)$. Note that the category $\operatorname{Ho}\left(\mathrm{Sp}_{G}^{O}\right)$ is equivalent to the Lewis-May $G-$ equivariant stable homotopy category of genuine $G$-spectra (see [33, Section IV.1]) introduced in [30].

As noted in the previous subsection, the model category $\operatorname{Sp}_{G}^{O}$ is stable, and hence the homotopy category $\mathrm{Ho}\left(\mathrm{Sp}_{G}^{O}\right)$ is naturally triangulated. Further, since the maps

$$
\lambda_{V}=\lambda_{0, V}: F_{V} S^{V} \longrightarrow F_{0} S^{0}
$$


are stable equivalences [33, Lemma III.4.5], it follows that the functor

$$
S^{V} \wedge-: \operatorname{Ho}\left(\mathrm{Sp}_{G}^{O}\right) \longrightarrow \mathrm{Ho}\left(\mathrm{Sp}_{G}^{O}\right)
$$

is an equivalence of categories for any finite-dimensional orthogonal $G$-representation $V$.

Next, before continuing, let us introduce the following notational convention. For any $G$-equivariant orthogonal spectra $X$ and $Y$, the abelian group $[X, Y]^{\mathrm{Ho}\left(\mathrm{Sp}_{G}^{O}\right)}$ of morphisms from $X$ to $Y$ in $\operatorname{Ho}\left(\operatorname{Sp}_{G}^{O}\right)$ will be denoted by $[X, Y]^{G}$.

An adjunction argument implies that, for any subgroup $H \leq G$ and an orthogonal $G$-spectrum $X$, there is a natural isomorphism

$$
\left[\Sigma_{+}^{\infty} G / H, X\right]_{*}^{G} \cong \pi_{*}^{H} X .
$$

As a consequence, we see that the set

$$
\left\{\Sigma_{+}^{\infty} G / H \mid H \leq G\right\}
$$

is a set of compact generators for the triangulated category $\operatorname{Ho}\left(\operatorname{Sp}_{G}^{O}\right)$; see Subsection 2.2. Note that, since $G$ is finite, for $*>0$ and any subgroups $H, H^{\prime} \leq G$, the abelian group

$$
\left[\Sigma_{+}^{\infty} G / H, \Sigma_{+}^{\infty} G / H^{\prime}\right]_{*}^{G}
$$

is finite; see eg [18, Proposition A.3].

Finally, recall from the introduction that the stable Burnside category, which is the full preadditive subcategory of $\operatorname{Ho}\left(\operatorname{Sp}_{G}^{O}\right)$ with objects the stable orbits $\Sigma_{+}^{\infty} G / H$ for $H \leq G$, is generated by the conjugations, transfers and restrictions. The stable Burnside category plays an important role in equivariant stable homotopy theory, as well as in representation theory. The contravariant functors from this category to abelian groups are exactly Mackey functors. Note that the stable Burnside category shows up in the formulation and proof of Theorem 1.1.1.

\section{Categorical input}

\subsection{Outline}

Recall that $G$ is a finite group. We start with the following:

Definition 3.1.1 Let $\mathscr{C}$ be a $G$-Top Todel $_{*}$-motegory (see Definition 2.1.4 and Subsection 2.3). Then $\mathscr{C}$ is said to be a $G$-equivariant stable model category if the adjunction

$$
S^{V} \wedge-: \mathscr{C} \rightleftarrows \mathscr{C}: \Omega^{V}(-)=(-)^{S^{V}}
$$


is a Quillen equivalence for any finite-dimensional orthogonal $G$-representation $V$.

Examples of $G$-equivariant stable model categories are the model category $\mathrm{Sp}_{G}^{O}$ of $G$-equivariant orthogonal spectra [33, Chapters II and III], the model category of $G$-equivariant orthogonal spectra equipped with the $\mathbb{S}$-model structure [48], the model category of $S_{G}$-modules [33, Section IV.2], the model category of $G$-equivariant continuous functors [5] and the model categories of $G$-equivariant topological symmetric spectra ([32] and [20]).

The following proposition is an equivariant version of [47, Theorem 3.8.2].

Proposition 3.1.2 Let $\mathscr{C}$ be a cofibrantly generated (see Definition 3.3.1), proper, $G$ equivariant stable model category. Then the category $\operatorname{Sp}_{G}^{O}(\mathscr{C})$ of internal orthogonal $G$-spectra in $\mathscr{C}$ (see Definition 3.2.1) possesses a $G$-equivariant stable model structure, and the $G-$ Top $_{*}$-adjunction

$$
\Sigma^{\infty}: \mathscr{C} \rightleftarrows \operatorname{Sp}_{G}^{O}(\mathscr{C}): \mathrm{Ev}_{0}
$$

is a Quillen equivalence.

The proof of this proposition is a straightforward equivariant generalization of the arguments in [47, Section 3.8]. It will occupy a significant part of this section.

The point of Proposition 3.1.2 is that one can replace (under some technical assumptions) any $G$-equivariant stable model category by a $G$-spectral one (see Definition 3.5.1), ie by an $\operatorname{Sp}_{G}^{O}$-model category. In particular, this implies that $\mathrm{Ho}(\mathscr{C})$ is tensored over the $G$-equivariant stable homotopy category $\mathrm{Ho}\left(\mathrm{Sp}_{G}^{O}\right)$.

To stress the importance of Proposition 3.1.2, we will now give a general strategy for how one should try to prove Conjecture 1.1.2. Recall that we are given a triangulated equivalence

$$
\Psi: \operatorname{Ho}\left(\mathrm{Sp}_{G}^{O}\right) \stackrel{\sim}{\longrightarrow} \operatorname{Ho}(\mathscr{C})
$$

with certain properties. By Proposition 3.1.2, there is a $G-$ Top $_{*}-$ Quillen equivalence

$$
\Sigma^{\infty}: \mathscr{C} \rightleftarrows \operatorname{Sp}_{G}^{O}(\mathscr{C}): \mathrm{Ev}_{0}
$$

Let $X$ be a cofibrant replacement of $\left(\mathbf{L} \Sigma^{\infty} \circ \Psi\right)(\mathbb{S})$. Since $\operatorname{Sp}_{G}^{O}(\mathscr{C})$ is $G$-spectral (see Definition 3.5.1), there is a $G-$ Top $_{*}-$ Quillen adjunction

$$
-\wedge X: \operatorname{Sp}_{G}^{O} \rightleftarrows \operatorname{Sp}_{G}^{O}(\mathscr{C}): \operatorname{Hom}(X,-) .
$$


Hence, in order to prove Conjecture 1.1.2, it suffices to show that the latter Quillen adjunction is a Quillen equivalence. Next, it follows from the assumptions on $\Psi$ that we have isomorphisms

$$
\Psi\left(\Sigma_{+}^{\infty} G / H\right) \cong \operatorname{REv}_{0}\left(\Sigma_{+}^{\infty} G / H \wedge^{\mathbf{L}} X\right)
$$

that are natural with respect to transfers, conjugations, and restrictions. Using these isomorphisms, we can choose an inverse of $\Psi$,

$$
\Psi^{-1}: \operatorname{Ho}(\mathscr{C}) \longrightarrow \operatorname{Ho}\left(\operatorname{Sp}_{G}^{O}\right),
$$

such that $\Psi^{-1}\left(\operatorname{REv}_{0}\left(\Sigma_{+}^{\infty} G / H \wedge^{\mathbf{L}} X\right)\right)=\Sigma_{+}^{\infty} G / H$. Moreover, since the isomorphisms above are natural with respect to the maps in the stable Burnside category, we get the identities

$$
\begin{aligned}
\Psi^{-1}\left(\mathbf{R E v}_{0}\left(g \wedge^{\mathbf{L}} X\right)\right) & =g, \\
\Psi^{-1}\left(\mathbf{R E v}_{0}\left(\operatorname{res}_{K}^{H} \wedge^{\mathbf{L}} X\right)\right) & =\operatorname{res}_{K}^{H}, \\
\Psi^{-1}\left(\mathbf{R E v}_{0}\left(\operatorname{tr}_{K}^{H} \wedge^{\mathbf{L}} X\right)\right) & =\operatorname{tr}_{K}^{H},
\end{aligned}
$$

where $g \in G$ and $K \leq H \leq G$. Now let us consider the composite

$$
F: \operatorname{Ho}\left(\operatorname{Sp}_{G}^{O}\right) \stackrel{-\wedge^{\mathbf{L}} X}{\longrightarrow} \operatorname{Ho}\left(\operatorname{Sp}_{G}^{O}(\mathscr{C})\right) \stackrel{\mathbf{R E v}_{0}}{\longrightarrow} \operatorname{Ho}(\mathscr{C}) \stackrel{\Psi^{-1}}{\longrightarrow} \operatorname{Ho}\left(\operatorname{Sp}_{G}^{O}\right)
$$

Since the functors $\mathbf{R} \mathrm{Ev}_{0}$ and $\Psi^{-1}$ are equivalences, to prove that $(-\wedge X, \operatorname{Hom}(X,-))$ is a Quillen equivalence corresponds to showing that the endofunctor

$$
F: \operatorname{Ho}\left(\operatorname{Sp}_{G}^{O}\right) \longrightarrow \operatorname{Ho}\left(\operatorname{Sp}_{G}^{O}\right)
$$

is an equivalence of categories. By the assumptions of Conjecture 1.1.2 and the properties of $\Psi^{-1}$, we see that $F$ enjoys the following properties:

(i) $F\left(\Sigma_{+}^{\infty} G / H\right)=\Sigma_{+}^{\infty} G / H$ for $H \leq G$.

(ii) $F$ preserves transfers, conjugations, and restrictions (and hence the stable Burnside category).

(iii) $F$ is an exact functor of triangulated categories and preserves infinite coproducts.

Similarly, if we start with the 2-localized genuine $G$-equivariant stable homotopy category $\operatorname{Ho}\left(\mathrm{Sp}_{G}^{O}\right.$,(2) $)$ and an equivalence $\mathrm{Ho}\left(\mathrm{Sp}_{G}^{O},{ }_{(2)}\right) \sim \operatorname{Ho}(\mathscr{C})$ as in the formulation of Theorem 1.1.1, we obtain an endofunctor $\operatorname{Ho}\left(\operatorname{Sp}_{G}^{O}\right.$,(2) $) \rightarrow \operatorname{Ho}\left(\operatorname{Sp}_{G}^{O}\right.$, (2) $)$ that also satisfies the properties (i), (ii) and (iii) above. The following theorem, which is one of the central results of this paper, immediately implies Theorem 1.1.1. 
Theorem 3.1.3 Let $G$ be a finite group and $F: \operatorname{Ho}\left(\operatorname{Sp}_{G}^{O},{ }_{(2)}\right) \rightarrow \operatorname{Ho}\left(\operatorname{Sp}_{G}^{O}\right.$,(2) $)$ an exact functor of triangulated categories that preserves arbitrary coproducts such that

$$
F\left(\Sigma_{+}^{\infty} G / H\right)=\Sigma_{+}^{\infty} G / H
$$

for $H \leq G$, and also

$$
F(g)=g, \quad F\left(\operatorname{res}_{K}^{H}\right)=\operatorname{res}_{K}^{H} \quad \text { and } \quad F\left(\operatorname{tr}_{K}^{H}\right)=\operatorname{tr}_{K}^{H}
$$

for $g \in G$ and $K \leq H \leq G$. Then $F$ is an equivalence of categories.

The proof of this theorem will be completed at the very end of this paper. In this section, we will concentrate on the proof of Proposition 3.1.2 and on the $p$-localization of the stable model category $\operatorname{Sp}_{G}^{O}$.

Let us outline the plan that will lead to the proof of Proposition 3.1.2. We first define the category $\operatorname{Sp}_{G}^{O}(\mathscr{C})$ of orthogonal $G$-spectra internal to a $G-\mathbf{T o p}_{*}$-model category $\mathscr{C}$ and discuss its categorical properties. Next, for any cofibrantly generated $G-$ Top $_{*}-$ model category $\mathscr{C}$, we construct the level model structure on $\operatorname{Sp}_{G}^{O}(\mathscr{C})$. Finally, using the same strategy as in [47], we establish the $G$-equivariant stable model structure on $\operatorname{Sp}_{G}^{O}(\mathscr{C})$ for any proper, cofibrantly generated, $G-\mathbf{T o p}_{*}$-model category $\mathscr{C}$ that is stable as an ordinary model category.

\subsection{Orthogonal $G$-spectra in equivariant model categories}

Recall the $G-\mathbf{T o p}_{*}$-category $O_{G}$ from Subsection 2.5. The objects of $O_{G}$ are finitedimensional orthogonal $G$-representations. For any finite-dimensional orthogonal $G$-representations $V$ and $W$, the pointed morphism $G$-space from $V$ to $W$ is the Thom space $O_{G}(V, W)$. Recall also that the category $\mathrm{Sp}_{G}^{O}$ is equivalent to the category of $O_{G}$-spaces (which is the category of $G-\mathbf{T o p}_{*}$-enriched functors from $O_{G}$ to $\left.G-\mathbf{T o p}_{*}\right)$.

Now suppose that $\mathscr{C}$ is a $G-\mathbf{T o p}_{*}-$ model category (in particular, $\mathscr{C}$ is pointed). We remind the reader that this means that we have tensors $K \wedge X$, cotensors $X^{K}$ and pointed mapping $G$-spaces $\operatorname{Map}(X, Y)$ for $K \in G$-Top $*$ and $X, Y \in \mathscr{C}$, that are related by adjunctions and satisfy certain properties; see Definition 2.1.4. In particular, the pushout-product axiom holds. Specifically, let $i: K \rightarrow L$ be a cofibration in the model category $G-\mathbf{T o p}_{*}$ and $j: A \rightarrow B$ a cofibration in the model category $\mathscr{C}$. Then the induced map

$$
i \square j: K \wedge B \vee_{K \wedge A} L \wedge A \longrightarrow L \wedge B
$$

is a cofibration in $\mathscr{C}$. Further, if either $i$ or $j$ is an acyclic cofibration, then so is $i \square j$. 
Definition 3.2.1 Let $\mathscr{C}$ be a $G$-Top ${ }_{*}$-model category. An orthogonal $G$-spectrum in $\mathscr{C}$ is a $G-\mathbf{T o p}_{*}$-enriched functor (see [28, Section 1.2]) from the category $O_{G}$ to $\mathscr{C}$.

The category of orthogonal $G$-spectra in $\mathscr{C}$ will be denoted by $\operatorname{Sp}_{G}^{O}(\mathscr{C})$. Note that by [33, Theorem II.4.3] (see also Subsection 2.5), the category $\operatorname{Sp}_{G}^{O}\left(G-\mathbf{T o p}_{*}\right)$ is equivalent to $\operatorname{Sp}_{G}^{O}$. Next, since $\mathscr{C}$ is complete and cocomplete, so is the category $\operatorname{Sp}_{G}^{O}(\mathscr{C})$ (see [28, Section 3.3]) and limits and colimits are constructed levelwise.

Remark 3.2.2 When talking about limits and colimits over $O_{G}$ or using the notations $\int_{V \in O_{G}}$ and $\int^{V \in O_{G}}$, we will always implicitly mean that the indexing category over which these (co)limits are taken is the chosen small skeleton sk $O_{G}$.

Proposition 3.2.3 Let $\mathscr{C}$ be a $G$-Top ${ }_{*}-$ model category. The category $\operatorname{Sp}_{G}^{O}(\mathscr{C})$ is enriched, tensored and cotensored over the symmetric monoidal category $\mathrm{Sp}_{G}^{O}$ of equivariant orthogonal $G$-spectra.

Proof Let $K \in \mathrm{Sp}_{G}^{O}$ and $X \in \mathrm{Sp}_{G}^{O}(\mathscr{C})$. We define $K \wedge X \in \mathrm{Sp}_{G}^{O}(\mathscr{C})$ by the following $G-$ Top $_{*}$-enriched coend:

$$
K \wedge X=\int^{V, W \in O_{G}} O_{G}(V \oplus W,-) \wedge K(V) \wedge X(W) .
$$

This product is unital and coherently associative. The proof uses the enriched Yoneda lemma [28, Section 3.10, (3.71)] and the Fubini theorem [28, Section 3.10, (3.63)]. We do not provide the details here as they are standard and well-known. Next, one defines cotensors by a $G-\mathbf{T o p}_{*}-$ enriched end

$$
X^{K}(V)=\int_{W \in O_{G}} X(W \oplus V)^{K(W)} .
$$

Finally, for any $X, Y \in \operatorname{Sp}_{G}^{O}(\mathscr{C})$, one can define Hom- $G$-spectra by a $G-\mathbf{T o p}_{*}-$ enriched end

$$
\operatorname{Hom}(X, Y)(V)=\int_{W \in O_{G}} \operatorname{Map}(X(W), Y(W \oplus V)) .
$$

It is a consequence of $[28$, Section $3.10,(3.71)]$ that these functors satisfy all the necessary adjointness properties:

$$
\operatorname{Hom}(K \wedge X, Y) \cong \operatorname{Hom}\left(X, Y^{K}\right) \cong \operatorname{Hom}(K, \operatorname{Hom}(X, Y)) .
$$




\subsection{The level model structure on $\operatorname{Sp}_{G}^{o}(\mathscr{C})$}

In order to establish the stable model structure on $\operatorname{Sp}_{G}^{O}(\mathscr{C})$, one needs the additional assumption that $\mathscr{C}$ is a cofibrantly generated $G-\mathbf{T o p}_{*}$-model category.

Definition 3.3.1 Let $\mathscr{C}$ be a $G-$ Top $_{*}$-model category. We say that $\mathscr{C}$ is a cofibrantly generated $G-$ Top $_{*}$-model category if there are sets $I$ and $J$ of maps in $\mathscr{C}$ such that the following hold:

(i) Let $A$ be the domain or codomain of a morphism from $I$. Then for any subgroup $H \leq G$ and any $n \geq 0$, the object

$$
\left(G / H \times D^{n}\right)_{+} \wedge A
$$

is small relative to $I$-cell (and hence relative to $I$-cof by [23, Proposition 2.1.16]).

(ii) Domains of morphisms in $J$ are small relative to $J$-cell and $I$-cell.

(iii) The class of fibrations is $J$-inj.

(iv) The class of acyclic fibrations is $I$-inj.

The model category $G-\mathbf{T o p}_{*}$ is a cofibrantly generated $G-\mathbf{T o p}_{*}-$ model category; see [33, Theorem III.1.8]. Other important examples of cofibrantly generated $G-$ Top $_{*}-$ model categories are the model category $\mathrm{Sp}_{G}^{O}$ of $G$-equivariant orthogonal spectra [33, Theorem III.4.2], the model category of $G$-equivariant orthogonal spectra equipped with the $\mathbb{S}$-model structure [48, Theorem 2.3.27], the model category of $S_{G}$-modules [33, Theorem IV.2.8], the model category of $G$-equivariant continuous functors [5, Theorem 1.3] and the model categories of $G$-equivariant topological symmetric spectra ([32] and [20]).

Remark 3.3.2 If a $G-$ Top $_{*}$-model category $\mathscr{C}$ is cofibrantly generated as an ordinary model category (see Definition 2.1.1), then it does not necessarily follow that $\mathscr{C}$ is a cofibrantly generated $G-$ Top $_{*}$-model category in the sense of Definition 3.3.1.

The conditions in Definition 3.3.1 are necessary for the proof of Proposition 3.4.7. In fact, all the claims in this section that come before Proposition 3.4.7 do not use the fact that $\mathscr{C}$ satisfies all the conditions of Definition 3.3.1. They still hold if we only assume that $\mathscr{C}$ is a $G-\mathbf{T o p}_{*}$-model category and cofibrantly generated as an underlying model category. However, for the rest of the paper, we will concentrate only on cofibrantly generated $G-$ Top $_{*}$-model categories in the sense of Definition 3.3.1 since more general model categories are irrelevant here. 
Now suppose that $\mathscr{C}$ is a cofibrantly generated $G-\mathbf{T o p}_{*}$-model category with $I$ and $J$ generating cofibrations and acyclic cofibrations.

Definition 3.3.3 Let $f: X \rightarrow Y$ be a morphism in $\operatorname{Sp}_{G}^{O}(\mathscr{C})$. The map $f$ is called a level equivalence if $f(V): X(V) \rightarrow Y(V)$ is a weak equivalence in $\mathscr{C}$ for any $V \in O_{G}$. It is called a level fibration if $f(V): X(V) \rightarrow Y(V)$ is a fibration in $\mathscr{C}$ for any $V \in O_{G}$. A map in $\operatorname{Sp}_{G}^{O}(\mathscr{C})$ is called a cofibration if it has the left lifting property with respect to all maps that are level fibrations and level equivalences (ie level acyclic fibrations).

The level model structure on $\operatorname{Sp}_{G}^{O}(\mathscr{C})$ that we will construct now is a cofibrantly generated model structure. Before stating the main proposition of this subsection, we would like to introduce the set of morphisms that will serve as generators of (acyclic) cofibrations in the level model structure on $\operatorname{Sp}_{G}^{O}(\mathscr{C})$.

The evaluation functor $\operatorname{Ev}_{V}: \operatorname{Sp}_{G}^{O}(\mathscr{C}) \rightarrow \mathscr{C}$, given by $X \mapsto X(V)$, has a left adjoint $G-$ Top $_{*}$-functor

$$
F_{V}: \mathscr{C} \longrightarrow \operatorname{Sp}_{G}^{O}(\mathscr{C})
$$

defined by

$$
F_{V} A=O_{G}(V,-) \wedge A
$$

For any finite-dimensional orthogonal $G$-representation $V$, consider the following sets of morphisms:

$$
F_{V} I=\left\{F_{V} i \mid i \in I\right\} \quad \text { and } \quad F_{V} J=\left\{F_{V} j \mid j \in J\right\} .
$$

We define

$$
F I=\bigcup_{V \in \operatorname{sk} O_{G}} F_{V} I \text { and } F J=\bigcup_{V \in \operatorname{sk} O_{G}} F_{V} J
$$

where sk $O_{G}$ denotes the chosen small skeleton of the category $O_{G}$.

The following proposition is an equivariant analog of [47, Proposition 3.7.2] (compare [19, Theorem 4.31]).

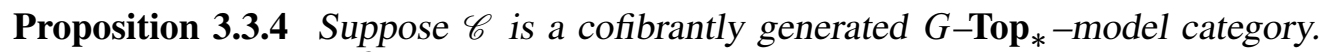
Then the category $\operatorname{Sp}_{G}^{O}(\mathscr{C})$ of orthogonal $G$-spectra in $\mathscr{C}$, together with the level equivalences, cofibrations and level fibrations described in Definition 3.3.3, forms a cofibrantly generated model category. The set FI generates cofibrations and the set FJ generates acyclic cofibrations.

Proof Illman's results [26, Theorem 7.1, Corollary 7.2] imply that, for any finitedimensional orthogonal $G$-representations $V$ and $W$, the space $O_{G}(V, W)$ is a $G-\mathrm{CW}$ complex. Since, for any object $A$ in $\mathscr{C}$,

$$
F_{V} A(W)=O_{G}(V, W) \wedge A,
$$


and the evaluation functors preserve colimits, it follows that any morphism in $F I-$ cell is a levelwise cofibration and any morphism in $F J$-cell is a levelwise acyclic cofibration. The rest of the proof is a verbatim translation of the proof of [47, Proposition 3.7.2] to our case, and we do not provide the details.

\subsection{The stable model structure on $\operatorname{Sp}_{G}^{O}(\mathscr{C})$}

This subsection establishes the stable model structure on $\operatorname{Sp}_{G}^{O}(\mathscr{C})$. For this one needs more assumptions than in Proposition 3.3.4. More precisely, we have to assume that the cofibrantly generated $G-\mathbf{T o p}_{*}$-model category $\mathscr{C}$ is proper and stable as an ordinary model category. The strategy is to follow the arguments given in [47, Section 3.8].

Let $W$ be a finite-dimensional orthogonal $G$-representation and

$$
\lambda_{W}=\lambda_{0, W}: F_{W} S^{W} \longrightarrow F_{0} S^{0}=\mathbb{S}
$$

denote the stable equivalence of $G$-equivariant orthogonal spectra that is adjoint to the identity map

$$
\text { id: } S^{W} \longrightarrow \operatorname{Ev}_{W}(\mathbb{S})=S^{W}
$$

see [33, Definition III.4.3, Lemma III.4.5] or Subsection 2.7.

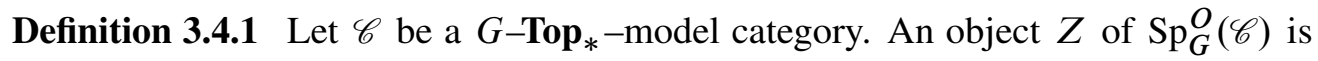
called an $\Omega$-spectrum if it is level fibrant and for any finite-dimensional orthogonal $G$-representation $W$, the induced map

$$
\lambda_{W}^{*}: Z \cong Z^{F_{0} S^{0}} \longrightarrow Z^{F_{W} S^{W}}
$$

is a level equivalence.

Since $Z^{F_{W} S^{W}} \cong Z(W \oplus-)^{S^{W}}$, this definition recovers the definition of a $G-$ $\Omega$-spectrum in the sense of [33, Definition III.3.1] when $\mathscr{C}=G$-Top ; $_{*}$; see also Definition 2.7.1.

Now suppose again that $\mathscr{C}$ is a cofibrantly generated $G-$ Top $_{*}-$ model category. By Proposition 3.3.4, the level model structure on $\operatorname{Sp}_{G}^{O}(\mathscr{C})$ is cofibrantly generated. Hence we can choose (and fix once and for all) a cofibrant replacement functor

$$
(-)^{c}: \operatorname{Sp}_{G}^{O}(\mathscr{C}) \longrightarrow \operatorname{Sp}_{G}^{O}(\mathscr{C}) .
$$

Definition 3.4.2 A morphism $f: A \rightarrow B$ in $\operatorname{Sp}_{G}^{O}(\mathscr{C})$ is a stable equivalence if, for any $\Omega$-spectrum $Z$, the map

$$
\operatorname{Hom}\left(f^{c}, Z\right): \operatorname{Hom}\left(B^{c}, Z\right) \longrightarrow \operatorname{Hom}\left(A^{c}, Z\right)
$$

is a level equivalence of $G$-equivariant orthogonal spectra. 
The following proposition is an equivariant analog of [47, Proposition 3.8.5]. Again we do not provide details here as the proof is completely analogous to the nonequivariant counterpart from [47].

Proposition 3.4.3 Let $\mathscr{C}$ be a left proper and cofibrantly generated $G$-Top - $_{*}$-model category. Suppose that $i: K \rightarrow L$ is a cofibration in $\operatorname{Sp}_{G}^{O}$ and $j: A \rightarrow B$ a cofibration in $\operatorname{Sp}_{G}^{O}(\mathscr{C})$. Then the pushout-product

$$
i \square j: K \wedge B \vee_{K \wedge A} L \wedge A \longrightarrow L \wedge B
$$

is a cofibration in $\operatorname{Sp}_{G}^{O}(\mathscr{C})$. The map $i \square j$ is also a stable equivalence if, in addition, $i$ or $j$ is a stable equivalence.

Next, we introduce the set $J_{\text {st }}$ which will serve as a set of generating acyclic cofibrations for the stable model structure on $\operatorname{Sp}_{G}^{O}(\mathscr{C})$ that we are going to establish. Let $W$ be a finite-dimensional orthogonal $G$-representation. Consider the levelwise mapping cylinder $M \lambda_{W}$ of the map $\lambda_{W}: F_{W} S^{W} \rightarrow F_{0} S^{0}$. The map $\lambda_{W}$ factors as a composite

$$
F_{W} S^{W} \stackrel{\kappa_{W}}{\longrightarrow} M \lambda_{W} \stackrel{r_{W}}{\longrightarrow} F_{0} S^{0},
$$

where $r_{W}$ is a $G$-equivariant homotopy equivalence and $\kappa_{W}$ a cofibration and a stable equivalence [33, Lemma III.4.5, Definition III.4.6]; see also Subsection 2.7. Define

$$
K=\left\{\kappa_{W} \square F_{V} i \mid V, W \in \operatorname{sk} O_{G}, i \in I\right\},
$$

where $\square$ is the pushout-product, $I$ is the fixed set of generating cofibrations in $\mathscr{C}$ (see Definition 3.3.1) and sk $O_{G}$ the fixed small skeleton of $O_{G}$. Next, recall from Proposition 3.3.4 that we have sets $F I$ and $F J$, generating cofibrations and acyclic cofibrations, respectively, in the level model structure. Define

$$
J_{\text {st }}=F J \cup K .
$$

For convenience we will denote the set $F I$ by $I_{\mathrm{st}}$. The cofibrations in the stable model structure on $\operatorname{Sp}_{G}^{O}(\mathscr{C})$ will be the same as in the level model structure, and thus $I_{\mathrm{st}}=F I$ will serve as a set of generating cofibrations for the stable model structure.

The following three propositions are again equivariant analogs of [47, Proposition 3.8.6, Lemma 3.8.7, Proposition 3.8.8]. For convenience we once again omit the proofs as they are very similar to those in [47].

Proposition 3.4.4 Let $\mathscr{C}$ be a left proper and cofibrantly generated $G$-Top - $_{*}$-model category. Then any morphism in $J_{\mathrm{st}}-$ cell is an $I_{\mathrm{st}}-$ cofibration (ie a cofibration) and a stable equivalence. 
Proposition 3.4.5 Let $\mathscr{C}$ be a cofibrantly generated $G$-Top - $_{*}$-model category and $X$ an object of $\operatorname{Sp}_{G}^{O}(\mathscr{C})$. Then the map $X \rightarrow *$ is $J_{\text {st }}$-injective if and only if $X$ is an $\Omega$-spectrum.

Proposition 3.4.6 Let $\mathscr{C}$ be a right proper and cofibrantly generated $G$-Top Todel $_{*}$-model category that is stable as an ordinary model category. Then a map in $\operatorname{Sp}_{G}^{O}(\mathscr{C})$ is $J_{\text {st }}-$ injective and a stable equivalence if and only if it is a level acyclic fibration.

Finally, we are ready to establish the stable model structure. The following proposition constructs the desired model structure. The proof that this model structure is stable is postponed to the next subsection.

Proposition 3.4.7 Let $\mathscr{C}$ be a proper and cofibrantly generated $G-$ Top $_{*}-$ model category that is stable as an ordinary model category. Then the category $\operatorname{Sp}_{G}^{O}(\mathscr{C})$ admits a cofibrantly generated model structure with stable equivalences as weak equivalences. The sets $I_{\mathrm{st}}$ and $J_{\text {st }}$ generate cofibrations and acyclic cofibrations, respectively. Furthermore, the fibrant objects are precisely the $\Omega$-spectra.

Proof The strategy of the proof is to verify the conditions of Proposition 2.1.2. The only things that still have to be checked are the smallness conditions. The rest follows from the previous three propositions.

That the domains of morphisms from $I_{\text {st }}$ are small relative to $I_{\mathrm{st}}-$ cell follows from the equality $I_{\mathrm{st}}=F I$ and Proposition 3.3.4. Next, recall that $J_{\mathrm{st}}=F J \cup K$. We will now verify that the domains of morphisms from $J_{\text {st }}$ are small relative to levelwise cofibrations. This will imply that the domains of morphisms in $J_{\text {st }}$ are small relative to $J_{\text {st }}$-cell since

$$
J_{\mathrm{st}}-\text { cell } \subset I_{\mathrm{st}}-\text { cof }
$$

by Proposition 3.4.4, and any morphism in $I_{\mathrm{st}}-$ cof is a levelwise cofibration as we saw in the proof of Proposition 3.3.4. That the domains of morphisms in $F J$ are small relative to levelwise cofibrations follows from an adjunction argument, Definition 3.3.1(ii) and [23, Proposition 2.1.16]. It remains to show that the domains of morphisms from $K$ are small relative to levelwise cofibrations. Any morphism in $K$ is a pushout-product of the form

$$
\kappa_{W} \square F_{V} i:\left(M \lambda_{W} \wedge F_{V} A\right) \vee_{F_{W}} S^{W} \wedge F_{V} A\left(F_{W} S^{W} \wedge F_{V} B\right) \longrightarrow M \lambda_{W} \wedge F_{V} B,
$$

where the morphism $i: A \rightarrow B$ is from the set $I$, and $V$ and $W$ are finite-dimensional orthogonal $G$-representations. For any finite $G-\mathrm{CW}$ complex $L$ and any object $D$ 
that is the domain or codomain of a map from $I$, the spectrum $F_{W} L \wedge F_{V} D$ is small relative to levelwise cofibrations. Indeed, we have an isomorphism

$$
\operatorname{Hom}\left(F_{W} L \wedge F_{V} D, X\right) \cong \operatorname{Map}(L \wedge D, X(V \oplus W)) .
$$

Since a pushout of small objects is small, Definition 3.3.1(i) implies that $L \wedge D$ is small with respect to $I$-cof, and hence $F_{W} L \wedge F_{V} D$ is small relative to levelwise cofibrations. Now we twice use that pushouts of small objects are small. First we conclude that $M \lambda_{W} \wedge F_{V} A$ is small relative to levelwise cofibrations and then we also see that

$$
\left(M \lambda_{W} \wedge F_{V} A\right) \vee_{F_{W}} S^{W} F_{V} A\left(F_{W} S^{W} \wedge F_{V} B\right)
$$

is small relative to levelwise cofibrations.

\section{5 $G$-equivariant stable model categories and completing the proof of Proposition 3.1.2}

We start with the following:

Definition 3.5.1 An $\mathrm{Sp}_{G}^{O}$-model category is called $G$-spectral. In other words, a model $\mathscr{C}$ category is $G$-spectral if it is enriched, tensored and cotensored over the model category $\operatorname{Sp}_{G}^{O}$, and the pushout-product axiom for tensors holds; see Definition 2.1.4.

By Proposition 2.7.4 the model category $\mathrm{Sp}_{G}^{O}$ is $G$-spectral. Next, Proposition 3.4.3 shows that the model structure of Proposition 3.4.7 on $\operatorname{Sp}_{G}^{O}(\mathscr{C})$ is $G$-spectral.

Recall from Definition 3.1.1 that a $G$-equivariant stable model category is a $G-\mathbf{T o p}_{*}-$ model category such that the Quillen adjunction

$$
S^{V} \wedge-: \mathscr{C} \rightleftarrows \mathscr{C}: \Omega^{V}(-)
$$

is a Quillen equivalence for any finite-dimensional orthogonal $G$-representation $V$. Before stating the next proposition, note that every $G$-spectral model category is obviously a $G-\mathbf{T o p}_{*}$-model category.

Proposition 3.5.2 Let $\mathscr{C}$ be a $G$-spectral model category. Then $\mathscr{C}$ is a $G$-equivariant stable model category.

Proof Consider the left Quillen functors

$$
S^{V} \wedge-: \mathscr{C} \longrightarrow \mathscr{C} \text { and } F_{V} S^{0} \wedge-: \mathscr{C} \longrightarrow \mathscr{C}
$$

and their derived functors

$$
S^{V} \wedge^{\mathbf{L}}-: \operatorname{Ho}(\mathscr{C}) \longrightarrow \operatorname{Ho}(\mathscr{C}) \text { and } F_{V} S^{0} \wedge^{\mathbf{L}}-: \operatorname{Ho}(\mathscr{C}) \longrightarrow \operatorname{Ho}(\mathscr{C}) \text {. }
$$


Since the map $\lambda_{V}: F_{V} S^{V} \rightarrow \mathbb{S}$ is a stable equivalence [33, Lemma III.4.5], for every cofibrant $X$ in $\mathscr{C}$, one has the weak equivalences

and

$$
S^{V} \wedge F_{V} S^{0} \wedge X \cong F_{V} S^{V} \wedge X \underset{\simeq}{\stackrel{\lambda_{V} \wedge 1}{\longrightarrow}} X
$$

$$
F_{V} S^{0} \wedge S^{V} \wedge X \cong F_{V} S^{V} \wedge X \underset{\simeq}{\stackrel{\lambda_{V} \wedge 1}{\longrightarrow}} X
$$

This implies that the functors $S^{V} \wedge^{\mathbf{L}}-$ and $F_{V} S^{0} \wedge^{\mathbf{L}}-$ are mutually inverse equivalences of categories.

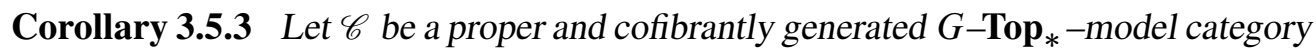
that is stable as an ordinary model category. Then the category $\operatorname{Sp}_{G}^{O}(\mathscr{C})$ together with the model structure of Proposition 3.4.7 is a $G$-equivariant stable model category.

From this point on, the model structure of Proposition 3.4.7 will be referred to as the

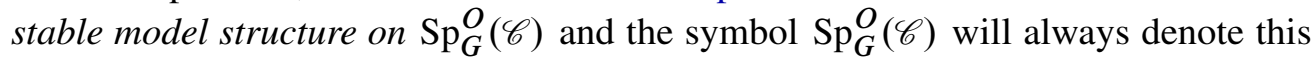
model category.

Finally, we observe that the $G-\mathbf{T o p}_{*}$-adjunction

$$
F_{0}=\Sigma^{\infty}: \mathscr{C} \rightleftarrows \operatorname{Sp}_{G}^{O}(\mathscr{C}): \mathrm{Ev}_{0}
$$

is a Quillen equivalence for every cofibrantly generated (in the sense of Definition 3.3.1) and proper $G$-equivariant stable model category $\mathscr{C}$. The proof of this fact is a verbatim translation of the last part of the proof of [47, Theorem 3.8.2] to our case. This finishes the proof of Proposition 3.1.2.

Remark 3.5.4 The Quillen equivalence

$$
\Sigma^{\infty}: \mathscr{C} \rightleftarrows \operatorname{Sp}_{G}^{O}(\mathscr{C}): \mathrm{Ev}_{0}
$$

is, in fact, a $G-\mathbf{T o p}_{*}$-Quillen equivalence. Indeed, $\left(\Sigma^{\infty}, \mathrm{Ev}_{0}\right)$ is a $G-\mathbf{T o p}_{*}$-enriched adjunction, and an enriched adjunction that is an underlying Quillen equivalence is an enriched Quillen equivalence by definition. Next, since enriched left adjoints preserve tensors [28, Sections 3.2, 3.7], the functor $\Sigma^{\infty}$ preserves tensors. Similarly, the right adjoint $\mathrm{Ev}_{0}$ preserves cotensors. Further, the equivalence

$$
\mathbf{L} \Sigma^{\infty}: \operatorname{Ho}(\mathscr{C}) \rightleftarrows \operatorname{Ho}\left(\operatorname{Sp}_{G}^{O}(\mathscr{C})\right): \mathbf{R} \mathrm{Ev}_{0}
$$

is a $\operatorname{Ho}\left(G-\mathbf{T o p}_{*}\right)$-enriched equivalence. Finally, we note that the functor $\mathbf{L} \Sigma^{\infty}$ preserves derived tensors, and since $\mathbf{R} \mathrm{Ev}_{0}$ is an inverse of $\mathbf{L} \Sigma^{\infty}$, it is also compatible with derived tensors. 


\subsection{The $p$-local model structure on $G$-equivariant orthogonal spectra}

This subsection reviews the $p$-localization of the stable model structure on $\operatorname{Sp}_{G}^{O}$ for any prime $p$. Note that one can construct the $p$-local model structure on $\mathrm{Sp}_{G}^{O}$ by using general localization techniques of [22] or [7]. Another possibility is to translate the arguments of [46, Section 4] to the equivariant context.

Definition 3.6.1 (i) A map $f: X \rightarrow Y$ of orthogonal $G$-spectra is called a $p$-local equivalence if the induced map

$$
\pi_{*}^{H}(f) \otimes \mathbb{Z}_{(p)}: \pi_{*}^{H} X \otimes \mathbb{Z}_{(p)} \longrightarrow \pi_{*}^{H} Y \otimes \mathbb{Z}_{(p)}
$$

is an isomorphism for any subgroup $H$ of $G$.

(ii) A map $f: X \rightarrow Y$ of orthogonal $G$-spectra is called a $p$-local fibration if it has the right lifting property with respect to all maps that are cofibrations and $p$-local equivalences.

Recall from Section 2 that the stable model structure on $\operatorname{Sp}_{G}^{O}$ is cofibrantly generated where $I_{\mathrm{st}}^{G}=I_{\mathrm{lv}}^{G}$ and $J_{\mathrm{st}}^{G}=K^{G} \cup J_{\mathrm{lv}}^{G}$ are generating cofibrations and acyclic cofibrations, respectively. Further, we also recall that the $\bmod l$ Moore space $M(l)$ is defined by the following pushout:

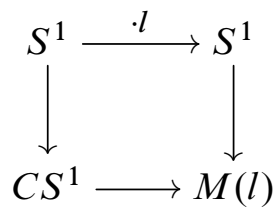

$(C(-)=(I, 0) \wedge-$ is the pointed cone functor.) Let $\iota: M(l) \rightarrow C M(l)$ denote the inclusion of $M(l)$ into the cone $C M(l)$. Define $J_{(p)}^{G}$ to be the set of maps of orthogonal $G$-spectra

$$
F_{n}\left(G / H_{+} \wedge \Sigma^{m} \iota\right): F_{n}\left(G / H_{+} \wedge \Sigma^{m} M(l)\right) \longrightarrow F_{n}\left(G / H_{+} \wedge \Sigma^{m} C M(l)\right),
$$

where $n, m \geq 0, H \leq G$ and $l$ is prime to $p$, ie invertible in $\mathbb{Z}_{(p)}$. We let $J_{\text {loc }}^{G}$ denote the union $J_{\text {st }}^{G} \cup J_{(p)}^{G}$.

Proposition 3.6.2 Let $G$ be a finite group and $p$ a prime. Then the category $\mathrm{Sp}_{G}^{O}$ of $G$-equivariant orthogonal spectra, together with $p$-local equivalences, cofibrations and $p$-local fibrations, forms a cofibrantly generated stable model category. The set $I_{\mathrm{st}}^{G}$ generates cofibrations and the set $J_{\text {loc }}^{G}$ generates acyclic cofibrations. Furthermore, the fibrant objects are precisely the $G-\Omega$-spectra whose $H$-equivariant homotopy groups are $p$-local for any $H \leq G$. 
Proof We do not give the details here and only observe that it is completely analogous to the proof in [46, Section 4].

Proposition 3.6.3 Suppose that $i: K \rightarrow L$ and $j: A \rightarrow B$ are cofibrations in $\operatorname{Sp}_{G}^{O},{ }_{(p)}$. Then the pushout-product

$$
i \square j: K \wedge B \vee_{K \wedge A} L \wedge A \longrightarrow L \wedge B
$$

is a cofibration in $\operatorname{Sp}_{G}^{O},{ }_{(p)}$. If, in addition, $i$ or $j$ is a $p$-local equivalence (ie a weak equivalence in $\mathrm{Sp}_{G}^{O},(p)$, then so is $i \square j$.

Proof By [23, Corollary 4.2.5], it suffices to check the claim for generating cofibrations and acyclic cofibrations. We do not give details because they are straightforward.

Since every stable equivalence of $G$-equivariant orthogonal spectra is a $p$-local equivalence, one obtains the following corollary:

Corollary 3.6.4 The model category $\mathrm{Sp}_{G}^{O}$, ${ }_{(p)}$ is $G$-spectral, ie an $\operatorname{Sp}_{G}^{O}$-model category (see Definition 3.5.1).

In view of Proposition 3.5.2, we also obtain the following:

Corollary 3.6.5 The model category $\mathrm{Sp}_{G}^{O},{ }_{(p)}$ is a $G$-equivariant stable model category (see Definition 3.1.1).

We end this subsection with some useful comments and remarks about the homotopy category $\mathrm{Ho}\left(\mathrm{Sp}_{G}^{O},{ }_{(p)}\right)$. Since the model category $\mathrm{Sp}_{G}^{O},{ }_{(p)}$ is stable, the homotopy category $\operatorname{Ho}\left(\operatorname{Sp}_{G}^{O},{ }_{(p)}\right)$ is naturally triangulated. Further, the following is a set of compact generators for $\mathrm{Ho}\left(\mathrm{Sp}_{G}^{O},{ }_{(p)}\right)$ :

$$
\left\{\Sigma_{+}^{\infty} G / H \mid H \leq G\right\} .
$$

This follows from the natural isomorphism

$$
\left[\Sigma_{+}^{\infty} G / H, X\right]_{*}^{\mathrm{Ho}\left(\mathrm{Sp}_{G}^{O},(p)\right)} \cong \pi_{*}^{H} X \otimes \mathbb{Z}_{(p)} .
$$

Finally, we note that for any $G$-equivariant orthogonal spectra $X$ and $Y$, the abelian group of morphisms $[X, Y]^{\mathrm{Ho}\left(\mathrm{Sp}_{G}^{O},{ }_{(p)}\right)}$ in $\mathrm{Ho}\left(\mathrm{Sp}_{G}^{O},{ }_{(p)}\right)$ (which will be also denoted by $[X, Y]^{G}$ abusing notation) is $p$-local. This follows from the fact that, for any integer $l$

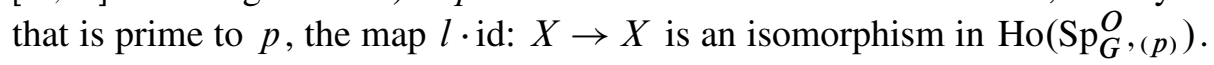




\subsection{Reduction to Theorem 3.1.3}

Now we are finally ready to explain why the arguments of Subsection 3.1 carry over to the $p$-local case.

Let $\mathscr{C}$ be a cofibrantly generated (in the sense of Definition 3.3.1), proper, $G$-equivariant stable model category. Suppose that

$$
\Psi: \operatorname{Ho}\left(\operatorname{Sp}_{G}^{O},(p)\right) \stackrel{\sim}{\longrightarrow} \operatorname{Ho}(\mathscr{C})
$$

is an equivalence of triangulated categories such that

$$
\Psi\left(\Sigma_{+}^{\infty} G / H\right) \cong G / H_{+} \wedge^{\mathbf{L}} \Psi(\mathbb{S})
$$

for any $H \leq G$. Suppose further that the latter isomorphisms are natural with respect to the restrictions, conjugations and transfers. By Proposition 3.1.2, there is a $G-\mathbf{T o p}_{*}-$ Quillen equivalence

$$
\Sigma^{\infty}: \mathscr{C} \rightleftarrows \operatorname{Sp}_{G}^{O}(\mathscr{C}): \mathrm{Ev}_{0}
$$

Next, as in Subsection 3.1, let $X$ be a cofibrant replacement of $\left(\mathbf{L} \Sigma^{\infty} \circ \Psi\right)(\mathbb{S})$. Since $\mathrm{Sp}_{G}^{O}(\mathscr{C})$ is $G$-spectral (see Proposition 3.4.3), there is a $G-\mathbf{T o p}_{*}-$ Quillen adjunction

$$
-\wedge X: \operatorname{Sp}_{G}^{O} \rightleftarrows \operatorname{Sp}_{G}^{O}(\mathscr{C}): \operatorname{Hom}(X,-)
$$

Since the Hom groups of $\operatorname{Ho}(\mathscr{C})$ are $p$-local, the latter Quillen adjunction yields a $G-$ Top $_{*}-$ Quillen adjunction

$$
-\wedge X: \operatorname{Sp}_{G}^{O},(p) \rightleftarrows \operatorname{Sp}_{G}^{O}(\mathscr{C}): \operatorname{Hom}(X,-) .
$$

Next, choose $\Psi^{-1}$ as in Subsection 3.1 and consider the composite

$$
F: \operatorname{Ho}\left(\operatorname{Sp}_{G}^{O},(p)\right) \stackrel{-\wedge^{\mathbf{L}} X}{\longrightarrow} \operatorname{Ho}\left(\operatorname{Sp}_{G}^{O}(\mathscr{C})\right) \stackrel{\mathbf{R E v}_{0}}{\longrightarrow} \operatorname{Ho}(\mathscr{C}) \stackrel{\Psi^{-1}}{\longrightarrow} \operatorname{Ho}\left(\operatorname{Sp}_{G}^{O},{ }_{(p)}\right) .
$$

Since the functors $\mathbf{R} \mathrm{Ev}_{0}$ and $\Psi^{-1}$ are equivalences, proving that $(-\wedge X, \operatorname{Hom}(X,-))$ is a Quillen equivalence corresponds to showing that the endofunctor

$$
F: \operatorname{Ho}\left(\mathrm{Sp}_{G}^{O},{ }_{(p)}\right) \longrightarrow \operatorname{Ho}\left(\mathrm{Sp}_{G}^{O},{ }_{(p)}\right)
$$

is an equivalence of categories. By the assumptions and the properties of $\Psi^{-1}$, we see that $F$ enjoys the following properties: 
(i) $F\left(\Sigma_{+}^{\infty} G / H\right)=\Sigma_{+}^{\infty} G / H$, for $H \leq G$.

(ii) $F(g)=g, F\left(\operatorname{res}_{K}^{H}\right)=\operatorname{res}_{K}^{H}$, and $F\left(\operatorname{tr}_{K}^{H}\right)=\operatorname{tr}_{K}^{H}$, for $g \in G$ and $K \leq H \leq G$.

(iii) $F$ is an exact functor of triangulated categories and preserves infinite coproducts.

So finally, we see that in order to prove Theorem 1.1.1, it suffices to prove Theorem 3.1.3. Note that we do not expect that an odd primary version of Theorem 3.1.3 is true. However, we still think that Conjecture 1.1.2 holds. Schwede's paper [43] suggests that the proof in the odd primary case should use the explicit construction of the endofunctor $F$, whereas in the 2-local case, certain axiomatic properties of $F$ are enough to get the desired result, as Theorem 3.1.3 shows. This is a generic difference between the 2-local case and the $p$-local case for $p$ an odd prime.

\section{Free $G$-spectra}

The set $\left\{\Sigma_{+}^{\infty} G / H \mid H \leq G\right\}$ is a set of compact generators for the triangulated category $\mathrm{Ho}\left(\operatorname{Sp}_{G}^{O},(2)\right)$. Hence, by Lemma 2.2.2, in order to prove Theorem 3.1.3 it suffices to show that, for any subgroups $H$ and $K$ of $G$, the map

$F:\left[\Sigma_{+}^{\infty} G / H, \Sigma_{+}^{\infty} G / K\right]_{*}^{G} \longrightarrow\left[F\left(\Sigma_{+}^{\infty} G / H\right), F\left(\Sigma_{+}^{\infty} G / K\right)\right]_{*}^{G}=\left[\Sigma_{+}^{\infty} G / H, \Sigma_{+}^{\infty} G / K\right]_{*}^{G}$ induced by $F$ is an isomorphism.

In this section we show that, under the assumptions of 3.1.3, the map

$$
F:\left[\Sigma_{+}^{\infty} G, \Sigma_{+}^{\infty} G\right]_{*}^{G} \longrightarrow\left[F\left(\Sigma_{+}^{\infty} G\right), F\left(\Sigma_{+}^{\infty} G\right)\right]_{*}^{G}=\left[\Sigma_{+}^{\infty} G, \Sigma_{+}^{\infty} G\right]_{*}^{G}
$$

is an isomorphism. In fact, we will not need all the assumptions of 3.1.3 to prove this. Since the functor $F$ sends $\Sigma_{+}^{\infty} G$ to itself, it restricts to an exact endofunctor of the localizing subcategory of $\operatorname{Ho}\left(\operatorname{Sp}_{G}^{O},{ }_{(2)}\right)$ generated by $\Sigma_{+}^{\infty} G$. We will prove that any exact endofunctor of the latter localizing subcategory that preserves infinite coproducts, the suspension spectrum $\Sigma_{+}^{\infty} G$, and the morphisms $g: \Sigma_{+}^{\infty} G \longrightarrow \Sigma_{+}^{\infty} G$ for any $g \in G$, is an equivalence of categories.

Note that the graded endomorphism ring $\left[\Sigma_{+}^{\infty} G, \Sigma_{+}^{\infty} G\right]_{*}^{G}$ is isomorphic to the graded group algebra $\pi_{*} \mathbb{S}[G]$, and the localizing subcategory generated by $\Sigma_{+}^{\infty} G$ in $\operatorname{Ho}\left(\operatorname{Sp}_{G}^{O}\right)$ is equivalent to $\operatorname{Ho}\left(\operatorname{Mod}-\Sigma_{+}^{\infty} G\right)$, where $\Sigma_{+}^{\infty} G$ is considered as the group ring spectrum of $G$.

We say that an object $X \in \operatorname{Ho}\left(\operatorname{Sp}_{G}^{O}\right)$ is a free $G$-spectrum if it is contained in the localizing subcategory generated by $\Sigma_{+}^{\infty} G$.

In the rest of the paper everything will be 2 -localized and hence we will mostly omit the subscript 2. 


\subsection{Cellular structures}

We start with the following

Definition 4.1.1 Let $R$ be an orthogonal ring spectrum, $X$ an $R$-module and $n$ and $m$ integers such that $n \leq m$. We say that $X$ admits a finite $(n, m)$-cell structure if there are sequences of distinguished triangles

$$
\bigvee_{I_{k}} \Sigma^{k-1} R \longrightarrow \operatorname{sk}_{k-1} X \longrightarrow \mathrm{sk}_{k} X \longrightarrow \bigvee_{I_{k}} \Sigma^{k} R
$$

in $\operatorname{Ho}(\operatorname{Mod}-R)$, for $k=n, n+1, \ldots, m$, such that the sets $I_{k}$ are finite, $\operatorname{sk}_{n-1} X=*$ and $\operatorname{sk}_{m} X=X$.

In other words, an $R$-module $X$ admits a finite $(n, m)$-cell structure if and only if it admits a structure of a finite $R$-cell complex with all possible cells in dimensions between $n$ and $m$.

Recall that there is a Quillen adjunction

$$
G_{+} \wedge-: \operatorname{Mod}-\mathbb{S} \rightleftarrows \operatorname{Mod}-\Sigma_{+}^{\infty} G: U
$$

and that $\left[\Sigma_{+}^{\infty} G, \Sigma_{+}^{\infty} G\right]_{*}^{G} \cong \pi_{*} \mathbb{S}[G]$. The following proposition can be considered as a 2-local naive equivariant version of [42, Lemma 4.1] (compare [9, Theorem 4.2]).

Proposition 4.1.2 Any $\alpha \in\left[\Sigma_{+}^{\infty} G, \Sigma_{+}^{\infty} G\right]_{n}{ }^{\mathrm{Ho}\left(\mathrm{Sp}_{G},{ }_{(2)}\right)}$, for $n \geq 8$, factors over an $\Sigma_{+}^{\infty} G$-module that admits a finite $(1, n-1)-$ cell structure.

Proof We will omit the subscript 2. Under the derived adjunction

$$
G_{+} \wedge^{\mathbf{L}}-: \operatorname{Ho}(\operatorname{Mod}-\mathbb{S}) \rightleftarrows \operatorname{Ho}\left(\operatorname{Mod}-\Sigma_{+}^{\infty} G\right): \mathbf{R} U
$$

the element $\alpha$ corresponds to some map $\tilde{\alpha}: \mathbb{S}^{n} \rightarrow \mathbf{R} U\left(\Sigma_{+}^{\infty} G\right) \cong \bigvee_{G} \mathbb{S}$. By the proof of [42, Lemma 4.1], for any $g \in G$, we have a factorization

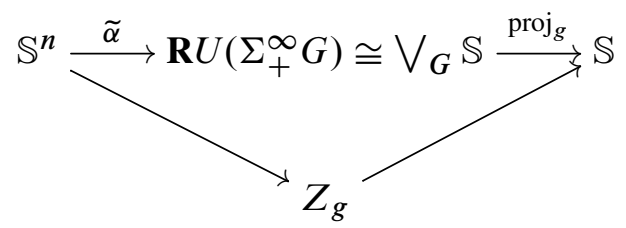

in the stable homotopy category, where $Z_{g}$ has $\mathbb{S}$-cells in dimensions between 1 and $n-1$. Here we use the fact that $n \geq 8$. Indeed, since $n \geq 8$, for any $g \in G$, the morphism $\operatorname{proj}_{g} \circ \tilde{\alpha}$ has an $\mathbb{F}_{2}-$ Adams filtration of at least 2 by the Hopf invariant one 
theorem [1], and hence one of the implications of [42, Lemma 4.1] applies to $\operatorname{proj}_{g} \circ \tilde{\alpha}$. Assembling these factorizations together, we get the following commutative diagram:

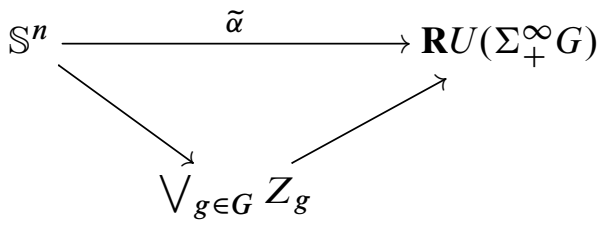

Finally, by adjunction, one obtains the following desired factorization:

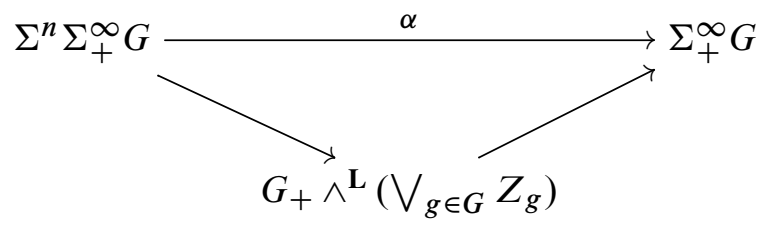

Next, we use Proposition 4.1.2 to prove the following important lemma.

Lemma 4.1.3 Let $G$ be a finite group and

$$
F: \operatorname{Ho}\left(\left(\operatorname{Mod}-\Sigma_{+}^{\infty} G\right)_{(2)}\right) \longrightarrow \operatorname{Ho}\left(\left(\operatorname{Mod}-\Sigma_{+}^{\infty} G\right)_{(2)}\right)
$$

an exact endofunctor such that

$$
F\left(\Sigma_{+}^{\infty} G\right)=\Sigma_{+}^{\infty} G
$$

and $F(g)=g$ in $\left[\Sigma_{+}^{\infty} G, \Sigma_{+}^{\infty} G\right]^{G}$ for any $g \in G$. Suppose that the map of graded rings

$$
F:\left[\Sigma_{+}^{\infty} G, \Sigma_{+}^{\infty} G\right]_{*}^{G} \longrightarrow\left[F\left(\Sigma_{+}^{\infty} G\right), F\left(\Sigma_{+}^{\infty} G\right)\right]_{*}^{G}=\left[\Sigma_{+}^{\infty} G, \Sigma_{+}^{\infty} G\right]_{*}^{G}
$$

is an isomorphism for $*$ below and including dimension $n$ for some $n \geq 0$. Then the following hold:

(i) Let $K$ and $L$ be $\Sigma_{+}^{\infty} G$-modules that admit finite $\left(\beta_{K}, \tau_{K}\right)-$ and $\left(\beta_{L}, \tau_{L}\right)-$-cell structures, respectively, and assume that $\tau_{K}-\beta_{L} \leq n$. Then the map

$$
F:[K, L]^{G} \longrightarrow[F(K), F(L)]^{G}
$$

is an isomorphism.

(ii) Let $K$ be an $\Sigma_{+}^{\infty} G$-module admitting a finite $\left(\beta_{K}, \tau_{K}\right)$-cell structure with $\tau_{K}-\beta_{K} \leq n+1$. Then there is an $\Sigma_{+}^{\infty} G$-module $K^{\prime}$ with a finite $\left(\beta_{K^{\prime}}, \tau_{K^{\prime}}\right)$ cell structure such that $\beta_{K} \leq \beta_{K^{\prime}}, \tau_{K^{\prime}} \leq \tau_{K}$ and $F\left(K^{\prime}\right) \cong K$. 
(iii) If $n+1 \geq 8$, then the map

$$
F:\left[\Sigma_{+}^{\infty} G, \Sigma_{+}^{\infty} G\right]_{n+1}^{G} \longrightarrow\left[\Sigma_{+}^{\infty} G, \Sigma_{+}^{\infty} G\right]_{n+1}^{G}
$$

is an isomorphism.

Proof Part (i) When $K$ and $L$ are both finite wedges of type $\bigvee \Sigma^{l_{0}} \Sigma_{+}^{\infty} G\left(l_{0}\right.$ is fixed), then the claim holds by assumption.

We start with the case when $L$ is a finite wedge of copies of $\Sigma^{l_{0}} \Sigma_{+}^{\infty} G$ for some integer $l_{0}$, and we proceed by induction on $\tau_{K}-\beta_{K}$. As already noted, the claim holds when $\tau_{K}-\beta_{K}=0$. Now suppose we are given $K$ with $\tau_{K}-\beta_{K}=r$ for $r \geq 1$, and assume that the claim holds for all $\Sigma_{+}^{\infty} G$-modules $M$ that have a finite $\left(\beta_{M}, \tau_{M}\right)$-cell structure with $\tau_{M}-\beta_{M}<r$. Consider the distinguished triangle

$$
\bigvee_{I_{\tau_{K}}} \Sigma^{\tau_{K}-1} \Sigma_{+}^{\infty} G \longrightarrow \mathrm{sk}_{\tau_{K}-1} K \longrightarrow K \longrightarrow \bigvee_{I_{\tau_{K}}} \Sigma^{\tau_{K}} \Sigma_{+}^{\infty} G
$$

The $\Sigma_{+}^{\infty} G$-module $\mathrm{sk}_{\tau_{K}-1} K$ has a finite $\left(\beta_{K}, \tau_{K}-1\right)$-cell structure. For convenience, let $P$ denote the wedge $\bigvee_{I_{\tau_{K}}} \Sigma^{\tau_{K}-1} \Sigma_{+}^{\infty} G$. The latter distinguished triangle induces a commutative diagram

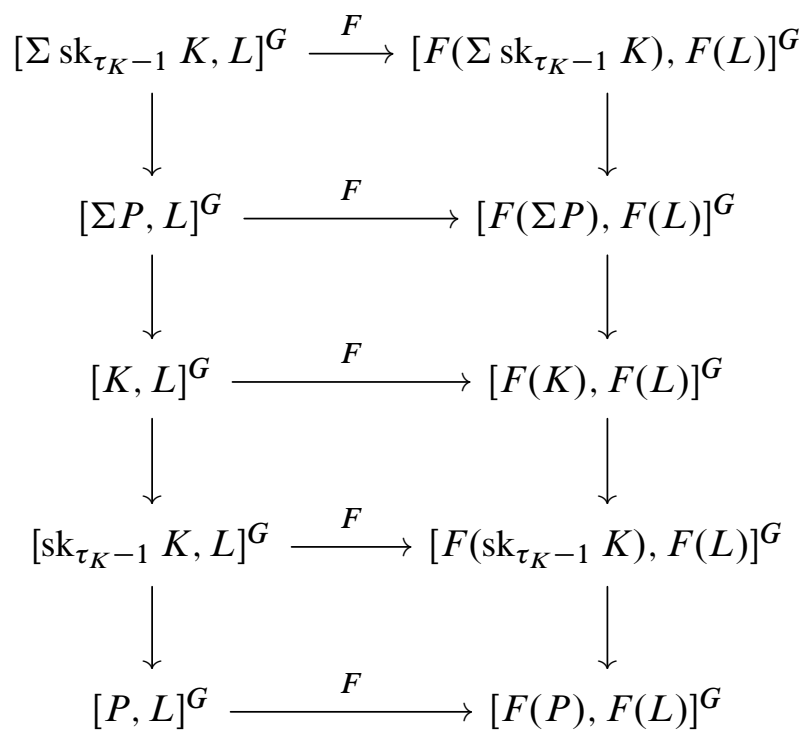

with exact columns (The functor $F$ is exact.). By the induction basis, the second and the last horizontal morphisms in this diagram are isomorphisms. The fourth morphism is an isomorphism by the induction assumption. Finally, since $\Sigma \mathrm{sk}_{\tau_{K}-1} K$ has a finite 
$\left(\beta_{K}+1, \tau_{K}\right)$-cell structure, the first horizontal map is also an isomorphism by the induction assumption. Hence, the claim follows by the five lemma.

Next, we do a similar induction with respect to $\tau_{L}-\beta_{L}$. The case $\tau_{L}-\beta_{L}=0$ is taken care of by the previous paragraph. For the inductive step we choose a distinguished triangle

$$
\mathrm{sk}_{\beta_{L}} L \longrightarrow L \longrightarrow L^{\prime} \longrightarrow \Sigma \mathrm{sk}_{\beta_{L}} L
$$

The octahedral axiom implies that the $\Sigma_{+}^{\infty} G$-module $L^{\prime}$ admits a finite $\left(\beta_{L}+1, \tau_{L}\right)$ cell structure. Now, as in the previous case, a five lemma argument finishes the proof.

Part (ii) We do induction on $\tau_{K}-\beta_{K}$. If $\tau_{K}-\beta_{K}=0$, then $K$ is stably equivalent to a finite wedge $\bigvee \Sigma^{l_{0}} \Sigma_{+}^{\infty} G$, for a fixed integer $l_{0}$, and the claim holds since $F\left(\Sigma_{+}^{\infty} G\right)=$ $\Sigma_{+}^{\infty} G$. For the induction step, choose a distinguished triangle

$$
\bigvee_{I_{\tau_{K}}} \Sigma^{\tau_{K}-1} \Sigma_{+}^{\infty} G \stackrel{\alpha}{\longrightarrow} \operatorname{sk}_{\tau_{K}-1} K \longrightarrow K \longrightarrow \bigvee_{I_{\tau_{K}}} \Sigma^{\tau_{K}} \Sigma_{+}^{\infty} G
$$

as above. By the induction assumption, there is an $\Sigma_{+}^{\infty} G$-module $M$ with a finite $\left(\beta_{M}, \tau_{M}\right)$-cell structure such that $\beta_{K} \leq \beta_{M}, \tau_{M} \leq \tau_{K}-1$ and $F(M) \cong \operatorname{sk}_{\tau_{K}-1} K$. Consider the composite

$$
F\left(\bigvee_{I_{\tau_{K}}} \Sigma^{\tau_{K}-1} \Sigma_{+}^{\infty} G\right) \stackrel{\cong}{\longrightarrow} \bigvee_{I_{\tau_{K}}} \Sigma^{\tau_{K}-1} \Sigma_{+}^{\infty} G \stackrel{\alpha}{\longrightarrow} \mathrm{sk}_{\tau_{K}-1} K \stackrel{\cong}{\longrightarrow} F(M) .
$$

Since $\tau_{K}-1-\beta_{M} \leq \tau_{K}-1-\beta_{K} \leq n$, part (i) yields that there exists

$$
\alpha^{\prime} \in\left[\bigvee_{I_{\tau_{K}}} \Sigma^{\tau_{K}-1} \Sigma_{+}^{\infty} G, M\right]^{G}
$$

such that $F\left(\alpha^{\prime}\right)$ equals the latter composition. Next, choose a distinguished triangle

$$
\bigvee_{I_{\tau_{K}}} \Sigma^{\tau_{K}-1} \Sigma_{+}^{\infty} G \stackrel{\alpha^{\prime}}{\longrightarrow} M \longrightarrow K^{\prime} \longrightarrow \bigvee_{I_{\tau_{K}}} \Sigma^{\tau_{K}} \Sigma_{+}^{\infty} G
$$

The $\Sigma_{+}^{\infty} G$-module $K^{\prime}$ has a finite $\left(\beta_{M}, \tau_{K}\right)$-cell structure. On the other hand, since $F$ is exact, one of the axioms for triangulated categories implies that there is a morphism $K \rightarrow F\left(K^{\prime}\right)$ that makes the diagram

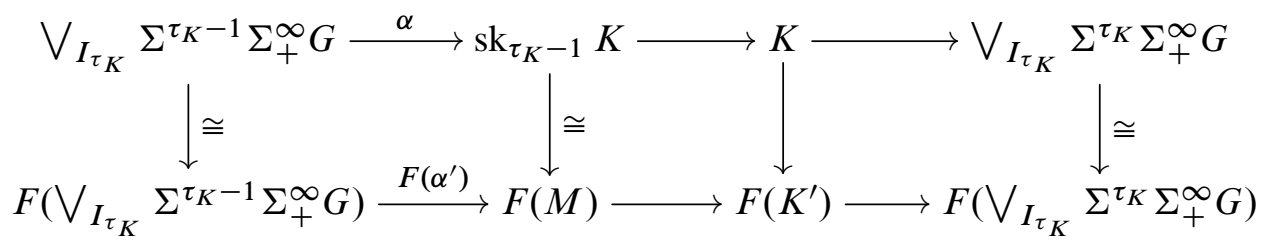


commute. Now another five lemma argument shows that, in fact, the map $K \rightarrow F\left(K^{\prime}\right)$ is an isomorphism in $\operatorname{Ho}\left(\operatorname{Mod}-\Sigma_{+}^{\infty} G\right)$, and thus the proof of part (ii) is completed.

Part (iii) By Proposition 4.1.2, any morphism $\alpha \in\left[\Sigma^{n+1} \Sigma_{+}^{\infty} G, \Sigma_{+}^{\infty} G\right]^{G}$ factors over some $\Sigma_{+}^{\infty} G$-module $K$ that has a finite $(1, n)$-cell structure. By part (ii), there exists an $\Sigma_{+}^{\infty} G$-module $K^{\prime}$ admitting a finite $\left(\beta_{K^{\prime}}, \tau_{K^{\prime}}\right)$-cell structure such that $1 \leq \beta_{K^{\prime}}$, $\tau_{K^{\prime}} \leq n$ and $F\left(K^{\prime}\right) \cong K$. Hence we get the following commutative diagram:

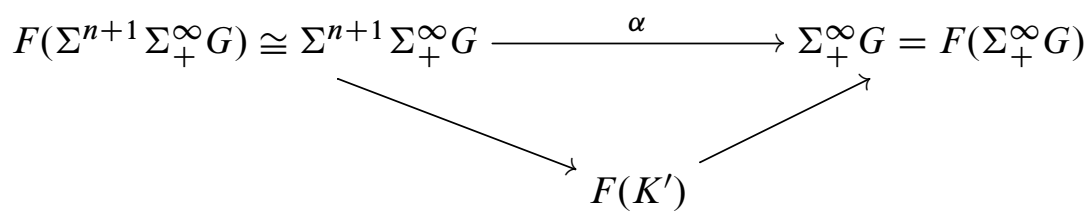

Since $n+1-\beta_{K^{\prime}} \leq n+1-1=n$ and $\tau_{K^{\prime}}-0=\tau_{K^{\prime}} \leq n$, part (i) implies that both maps in the latter factorization are in the image of $F$. Hence, the map $\alpha$ is also in the image of the functor $F$, yielding that

$$
F:\left[\Sigma_{+}^{\infty} G, \Sigma_{+}^{\infty} G\right]_{n+1}^{G} \longrightarrow\left[\Sigma_{+}^{\infty} G, \Sigma_{+}^{\infty} G\right]_{n+1}^{G}
$$

is surjective. As the source and target of this morphism are finite of the same cardinality, we conclude that it is an isomorphism.

From Lemma 4.1.3, together with Lemma 2.2.2, we have the following:

Corollary 4.1.4 Let $F$ be as in 4.1.3 and assume that it commutes with infinite coproducts. If the morphism

$$
F:\left[\Sigma_{+}^{\infty} G, \Sigma_{+}^{\infty} G\right]_{*}^{G} \longrightarrow\left[\Sigma_{+}^{\infty} G, \Sigma_{+}^{\infty} G\right]_{*}^{G}
$$

is an isomorphism for $* \leq 7$, then the functor $F$ is an equivalence of categories.

\subsection{Taking care of the dimensions $\leq 7$}

For this subsection,

$$
F: \operatorname{Ho}\left(\left(\operatorname{Mod}-\Sigma_{+}^{\infty} G\right)_{(2)}\right) \longrightarrow \operatorname{Ho}\left(\left(\operatorname{Mod}-\Sigma_{+}^{\infty} G\right)_{(2)}\right)
$$

again denotes an exact endofunctor that commutes with infinite coproducts such that

$$
F\left(\Sigma_{+}^{\infty} G\right)=\Sigma_{+}^{\infty} G
$$

and $F(g)=g$ in $\left[\Sigma_{+}^{\infty} G, \Sigma_{+}^{\infty} G\right]^{G}$ for any $g \in G$. We will now show that the map

$$
F:\left[\Sigma_{+}^{\infty} G, \Sigma_{+}^{\infty} G\right]_{*}^{G} \longrightarrow\left[F\left(\Sigma_{+}^{\infty} G\right), F\left(\Sigma_{+}^{\infty} G\right)\right]_{*}^{G}=\left[\Sigma_{+}^{\infty} G, \Sigma_{+}^{\infty} G\right]_{*}^{G}
$$


is an isomorphism for $* \leq 7$. By Corollary 4.1.4 this will imply that the functor $F$ is a self equivalence of the category of free $G$-spectra.

Recall that we have a preferred isomorphism $\left[\Sigma_{+}^{\infty} G, \Sigma_{+}^{\infty}\right]_{*}^{G} \cong \pi_{*} \mathbb{S}[G]$. Since $F(g)=g$ for any $g \in G$, and since the map $F: \pi_{*} \mathbb{S}[G] \longrightarrow \pi_{*} \mathbb{S}[G]$ is a ring homomorphism, we conclude that it is an isomorphism for $*=0$. Note that $\pi_{*} \mathbb{S}[G]$ is finite for $*>0$ and the Hopf maps $\eta, v$ and $\sigma$ multiplicatively generate $\pi_{* \leq 7} \mathbb{S}$. Hence, it suffices to show that the Hopf maps (considered as elements of $\pi_{*} \mathbb{S}[G]$ via the unit map $\mathbb{S} \rightarrow \Sigma_{+}^{\infty} G$ ) are in the image of $F$.

We start by showing that $F(\eta)=\eta$. Recall that the mod 2 Moore spectrum $M(2)$ in the 2-localized (nonequivariant) stable homotopy category is defined by the distinguished triangle

$$
\mathbb{S} \stackrel{2}{\longrightarrow} \mathbb{S} \stackrel{\iota}{\longrightarrow} M(2) \stackrel{\partial}{\longrightarrow} \mathbb{S}^{1}
$$

and that the map 2: $M(2) \longrightarrow M(2)$ factors as a composite

$$
M(2) \stackrel{\partial}{\rightarrow} \mathbb{S}^{1} \stackrel{\eta}{\longrightarrow} \mathbb{S} \stackrel{\iota}{\longrightarrow} M(2) .
$$

Applying the functor $G_{+} \wedge^{\mathbf{L}}-: \operatorname{Ho}(\operatorname{Mod}-\mathbb{S}) \longrightarrow \operatorname{Ho}\left(\operatorname{Mod}-\Sigma_{+}^{\infty} G\right)$ to the distinguished triangle above gives us this distinguished triangle

$$
\Sigma_{+}^{\infty} G \stackrel{2}{\longrightarrow} \Sigma_{+}^{\infty} G \stackrel{1 \wedge \iota}{\longrightarrow} G_{+} \wedge M(2) \stackrel{1 \wedge \partial}{\longrightarrow} \Sigma \Sigma_{+}^{\infty} G
$$

in $\operatorname{Ho}\left(\operatorname{Mod}-\Sigma_{+}^{\infty} G\right)$. Further, the map 2: $G_{+} \wedge M(2) \longrightarrow G_{+} \wedge M(2)$ factors as

$$
G_{+} \wedge M(2) \stackrel{1 \wedge \partial}{\longrightarrow} \Sigma \Sigma_{+}^{\infty} G \stackrel{\eta}{\longrightarrow} \Sigma_{+}^{\infty} G \stackrel{1 \wedge \iota}{\longrightarrow} G_{+} \wedge M(2) .
$$

One of the axioms for triangulated categories implies that we can choose an isomorphism

$$
F\left(G_{+} \wedge M(2)\right) \cong G_{+} \wedge M(2)
$$

so that the diagram

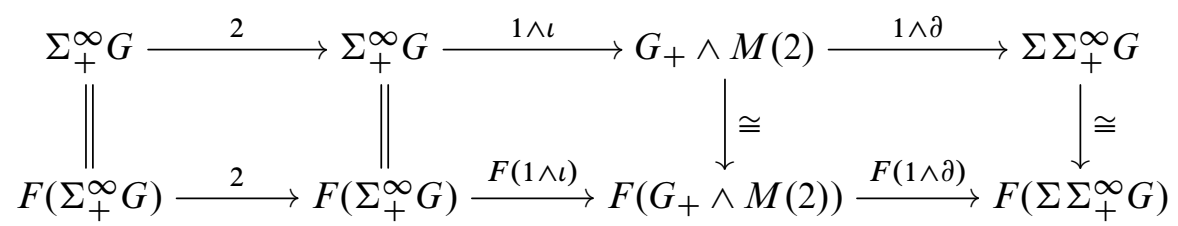

commutes. We fix the latter isomorphism once and for all and identify $F\left(G_{+} \wedge M(2)\right)$ with $G_{+} \wedge M(2)$. Note that, under this identification, the morphisms $F(1 \wedge \iota)$ and 
$F(1 \wedge \partial)$ correspond to $1 \wedge \iota$ and $1 \wedge \partial$, respectively. Next, since $F(2)=2$ and $2=(1 \wedge \iota) \eta(1 \wedge \partial)$, one gets the identity

$$
(1 \wedge \iota) F(\eta)(1 \wedge \partial)=2
$$

It is well known that the map 2: $M(2) \longrightarrow M(2)$ is nonzero; see [44, Proposition 4] (in fact, $[M(2), M(2)] \cong \mathbb{Z} / 4)$. Hence, $2: G_{+} \wedge M(2) \longrightarrow G_{+} \wedge M(2)$ is nonzero as there is a preferred ring isomorphism

$$
\left[G_{+} \wedge M(2), G_{+} \wedge M(2)\right]_{*}^{G} \cong[M(2), M(2)]_{*} \otimes \mathbb{Z}[G] .
$$

It now follows that $F(\eta) \neq 0$. Suppose $F(\eta)=\sum_{g \in A} \eta g$, where $A$ is a nonempty subset of $G$. We want to show that $A=\{1\}$. The identity $(1 \wedge \iota) F(\eta)(1 \wedge \partial)=2$ yields

$$
2=(1 \wedge \iota)\left(\sum_{g \in A} \eta g\right)(1 \wedge \partial)=\sum_{g \in A}(1 \wedge \iota) \eta(1 \wedge \partial) g=\sum_{g \in A} 2 g
$$

Once again using the isomorphism $\left[G_{+} \wedge M(2), G_{+} \wedge M(2)\right]_{*}^{G} \cong[M(2), M(2)]_{*} \otimes \mathbb{Z}[G]$ and the fact that $2 \neq 0$, we conclude that $A=\{1\}$, and hence $F(\eta)=\eta$.

Next, we show that $v$ is in the image of $F$. Let

$$
F(v)=m v+\sum_{g \in G \backslash\{1\}} n_{g} g v .
$$

Recall that, 2-locally, we have the identity (see eg [49, Theorem 14.1(i)])

$$
\eta^{3}=4 v
$$

Since $F(\eta)=\eta$, after applying $F$ to this identity, one obtains

$$
4 v=\eta^{3}=F\left(\eta^{3}\right)=F(4 v)=4 m v+\sum_{g \in G \backslash\{1\}} 4 n_{g} g v .
$$

As the element $v$ is a generator of the group $\pi_{3} \mathbb{S}_{(2)} \cong \mathbb{Z} / 8$, we conclude that $m=2 k+1$ for some $k \in \mathbb{Z}$, and for any $g \in G \backslash\{1\}$ we have $n_{g}=2 l_{g}$ and $l_{g} \in \mathbb{Z}$. Hence

$$
F(v)=(2 k+1) v+\sum_{g \in G \backslash\{1\}} 2 l_{g} g v .
$$

Using that $F(g)=g$, we also deduce that

$$
F\left(g_{0} v\right)=(2 k+1) g_{0} v+\sum_{g \in G \backslash\{1\}} 2 l_{g} g_{0} g v
$$


for any fixed $g_{0} \in G \backslash\{1\}$. Thus the image of $F$ in $\pi_{3} \mathbb{S}_{(2)}[G] \cong \bigoplus_{G} \mathbb{Z} / 8$ is additively generated by the rows of a $|G| \times|G|-$ matrix of the form

$$
\left(\begin{array}{ccc}
2 k+1 & & \text { even } \\
& \ddots & \\
\text { even } & & 2 k+1
\end{array}\right)
$$

where each diagonal entry is equal to $2 k+1$ and all the other entries are even. Since the determinant of this matrix is odd and hence a unit in $\mathbb{Z} / 8$, the homomorphism $F: \pi_{3} \mathbb{S}_{(2)}[G] \rightarrow \pi_{3} \mathbb{S}_{(2)}[G]$ is an isomorphism, and hence the element $v$ is in the image of $F$.

Finally, it remains to show that $\sigma \in \pi_{7} \mathbb{S} \subset \pi_{7} \mathbb{S}[G]$ is in the image of $F$. Recall that $\sigma$ is a generator of $\pi_{7} \mathbb{S}_{(2)} \cong \mathbb{Z} / 16$. We use the Toda bracket relation

$$
8 \sigma=\langle v, 8, v\rangle \quad \text { in } \pi_{*} \mathbb{S}_{(2)}
$$

(see eg [49, Lemmas 5.13 and 5.14]) that holds without indeterminacy as $\pi_{4} \mathbb{S}=0$. This implies that

$$
8 \sigma=\langle\nu, 8, v\rangle \quad \text { in } \pi_{*} \mathbb{S}[G] .
$$

Now since $F$ is an exact functor, one obtains

$$
8 F(\sigma)=\langle F(v), 8, F(v)\rangle
$$

Recall that

$$
F(v)=(2 k+1) v+\sum_{g \in G \backslash\{1\}} 2 l_{g} g v
$$

Let $F(\sigma)=m \sigma+\sum_{g \in G \backslash\{1\}} n_{g} g \sigma$. By [4, Theorem 1.3] and the relation $16 \sigma=0$, we get

$$
\begin{aligned}
8\left(m \sigma+\sum_{g \in G \backslash\{1\}} n_{g} g \sigma\right) & =\left\langle(2 k+1) v+\sum_{g \in G \backslash\{1\}} 2 l_{g} g v, 8,(2 k+1) v+\sum_{g \in G \backslash\{1\}} 2 l_{g} g v\right\rangle \\
& =8(2 k+1)^{2} \sigma .
\end{aligned}
$$

Hence we see that $m$ is odd and the numbers $n_{g}$ are even. Now a similar argument as in the case of $v$ implies that $F: \pi_{7} \mathbb{S}[G] \longrightarrow \pi_{7} \mathbb{S}[G]$ is surjective, and hence $\sigma$ is in the image of $F$.

By combining the results of this subsection with Corollary 4.1.4, we conclude that, under the assumptions of Theorem 3.1.3, the functor $F: \operatorname{Ho}\left(\operatorname{Sp}_{G,(2)}^{O}\right) \rightarrow \operatorname{Ho}\left(\operatorname{Sp}_{G,(2)}^{O}\right)$ becomes an equivalence when restricted to the full subcategory of free $G$-spectra, 
or equivalently, when restricted to $\operatorname{Ho}\left(\left(\operatorname{Mod}-\Sigma_{+}^{\infty} G\right)_{(2)}\right)$. In fact, we have proved the following more general proposition.

Proposition 4.2.1 Let $G$ be any finite group and

$$
F: \operatorname{Ho}\left(\left(\operatorname{Mod}-\Sigma_{+}^{\infty} G\right)_{(2)}\right) \longrightarrow \operatorname{Ho}\left(\left(\operatorname{Mod}-\Sigma_{+}^{\infty} G\right)_{(2)}\right)
$$

an exact endofunctor that preserves arbitrary coproducts such that

$$
F\left(\Sigma_{+}^{\infty} G\right)=\Sigma_{+}^{\infty} G
$$

and $F(g)=g$ in $\left[\Sigma_{+}^{\infty} G, \Sigma_{+}^{\infty} G\right]^{G}$ for all $g \in G$. Then $F$ is an equivalence of categories.

\section{Double coset embedding}

In this section, we will show that the graded maps between the generators $\left\{\Sigma_{+}^{\infty} G / H \mid\right.$ $H \leq G\}$ of $\mathrm{Ho}\left(\mathrm{Sp}_{G}^{O}\right)$ can be embedded into a certain direct sum indexed on a set of double cosets. This will be very useful in the proof of Theorem 3.1.3.

\subsection{Formulation}

Let $G$ be a finite group with subgroups $H$ and $K$. For the rest of this section, we fix once and for all a set $\{g\}$ of double coset representatives for $K \backslash G / H$. Recall that for any $g \in G$, the conjugated subgroup $\mathrm{gHg}^{-1}$ is denoted by ${ }^{g} H$. Further,

$$
\kappa_{g}:\left[\Sigma_{+}^{\infty} G / H, \Sigma_{+}^{\infty} G / K\right]_{*}^{G} \longrightarrow\left[\Sigma_{+}^{\infty} G /\left({ }^{g} H \cap K\right), \Sigma_{+}^{\infty} G /\left({ }^{g} H \cap K\right)\right]_{*}^{G}
$$

will stand for the map which is defined by the following commutative diagram:

$$
\begin{aligned}
& {\left[\Sigma_{+}^{\infty} G / H, \Sigma_{+}^{\infty} G / K\right]_{*}^{G} \stackrel{\kappa_{g}}{\longrightarrow}\left[\Sigma_{+}^{\infty} G /\left({ }^{g} H \cap K\right), \Sigma_{+}^{\infty} G /\left({ }^{g} H \cap K\right)\right]_{*}^{G}} \\
& g^{*} \downarrow \quad \uparrow\left(\operatorname{res}_{g_{H \cap K}}^{g_{H}}\right)^{*} \\
& {\left[\Sigma_{+}^{\infty} G /{ }^{g} H, \Sigma_{+}^{\infty} G / K\right]_{*}^{G} \stackrel{\left(\operatorname{tr}_{g_{H}}^{K} \cap K\right.}{{ }^{*}}\left[\Sigma_{+}^{\infty} G /{ }^{g} H, \Sigma_{+}^{\infty} G /\left({ }^{g} H \cap K\right)\right]_{*}^{G}}
\end{aligned}
$$

The aim of this section is to prove:

Proposition 5.1.1 The map

$$
\left[\Sigma_{+}^{\infty} G / H, \Sigma_{+}^{\infty} G / K\right]_{*}^{G} \stackrel{\left(\kappa_{g}\right)_{[g] \in K \backslash G / H}}{\longrightarrow} \bigoplus_{[g] \in K \backslash G / H}\left[\Sigma_{+}^{\infty} G /\left({ }^{g} H \cap K\right), \Sigma_{+}^{\infty} G /\left({ }^{g} H \cap K\right)\right]_{*}^{G}
$$

is a split monomorphism. 
The author suspects that this statement is known to the experts. However, since we were unable to find a reference, we decided to provide a detailed proof here. The proof is mainly based on the equivariant Spanier-Whitehead duality (see [30, Sections III.2 and V.9] and [35, Section XVI.7]) and on a combinatorial analysis of certain pointed $G$-sets.

\subsection{Induction and coinduction}

Let $H$ be a subgroup of $G$ with inclusion $i: H \hookrightarrow G$. The class of all finite-dimensional orthogonal $H$-representations of the form $i^{*} V$, where $V$ is a finite-dimensional orthogonal $G$-representation, contains the trivial representation and is closed under direct sums. Hence, according to [33, Section II.2, Theorem III.4.2], there is a stable model category $\mathrm{Sp}_{H \leq G}^{O}$ of $H$-equivariant orthogonal spectra indexed on the class of such representations (compare Subsection 2.7). Since finite-dimensional orthogonal $H$-representations that come from $G$-representations are cofinal in the class of all finite-dimensional orthogonal $H$-representations, [33, Remark V.1.10] implies that the Quillen adjunction

$$
\text { id: } \mathrm{Sp}_{H \leq G}^{O} \rightleftarrows \mathrm{Sp}_{H}^{O}: \mathrm{id}
$$

is a Quillen equivalence. Next, recall that there is a Quillen adjunction

$$
G \ltimes_{H}-: \operatorname{Sp}_{H \leq G}^{O} \rightleftarrows \operatorname{Sp}_{G}^{O}: \operatorname{Res}_{H}^{G},
$$

where $\left(G \ltimes_{H} X\right)(V)=G_{+} \wedge_{H} X\left(i^{*} V\right)$ for any $X \in \operatorname{Sp}_{H \leq G}^{O}$ and any finite-dimensional orthogonal $G$-representation $V$; see [33, Lemma V.2.2]. The functor $\operatorname{Res}_{H}^{G}$ is just the restriction along the map $i: H \hookrightarrow G$. In fact, the functor $\operatorname{Res}_{H}^{G}$ preserves weak equivalences, and moreover, it is also a left Quillen functor as we see from the Quillen adjunction

$$
\operatorname{Res}_{H}^{G}: \operatorname{Sp}_{G}^{O} \rightleftarrows \operatorname{Sp}_{H \leq G}^{O}: \operatorname{Map}_{H}\left(G_{+},-\right) .
$$

The right adjoint is defined by $\operatorname{Map}_{H}\left(G_{+}, X\right)(V)=\operatorname{Map}_{H}\left(G_{+}, X\left(i^{*} V\right)\right)$. Now since the functor id: $\mathrm{Sp}_{H \leq G}^{O} \rightarrow \mathrm{Sp}_{H}^{O}$ is a left Quillen functor, we also get a Quillen adjunction

$$
\operatorname{Res}_{H}^{G}: \operatorname{Sp}_{G}^{O} \rightleftarrows \operatorname{Sp}_{H}^{O}: \operatorname{Map}_{H}\left(G_{+},-\right)
$$

These Quillen adjunctions induce corresponding adjunctions on the derived level:

$$
G \ltimes_{H}-: \operatorname{Ho}\left(\operatorname{Sp}_{H}^{O}\right) \sim \operatorname{Ho}\left(\operatorname{Sp}_{H \leq G}^{O}\right) \rightleftarrows \operatorname{Ho}\left(\operatorname{Sp}_{G}^{O}\right): \operatorname{Res}_{H}^{G}
$$

and

$$
\operatorname{Res}_{H}^{G}: \operatorname{Ho}\left(\operatorname{Sp}_{G}^{O}\right) \rightleftarrows \operatorname{Ho}\left(\operatorname{Sp}_{H}^{O}\right): \operatorname{Map}_{H}\left(G_{+},-\right)
$$


Here we slightly abuse notation by denoting point-set level functors and their associated derived functors with the same symbols. Next, note that the equivalence

$$
\operatorname{Ho}\left(\operatorname{Sp}_{H}^{O}\right) \sim \operatorname{Ho}\left(\operatorname{Sp}_{H \leq G}^{O}\right)
$$

is a preferred one and is induced from the Quillen equivalence at the very beginning of this subsection.

The adjunctions recalled here are, in fact, special instances of the "change of groups" and "change of universe" functors of [33, Chapter V]. The functor $G \ltimes_{H}-$ is usually called the induction and the functor $\operatorname{Map}_{H}\left(G_{+},-\right)$is called the coinduction.

Now let $G \ltimes_{H} X$ denote the balanced product $G_{+} \wedge_{H} X$ for any pointed $G$-set (space) $X$. Consider the natural point-set level map

$$
w_{H}: G \ltimes_{H} X \longrightarrow \operatorname{Map}_{H}\left(G_{+}, X\right)
$$

given by

$$
w_{H}([g, x])(\gamma)= \begin{cases}\gamma g x & \text { if } \gamma g \in H \\ * & \text { if } \gamma g \notin H\end{cases}
$$

We remind the reader of the following result due to Wirthmüller:

Proposition 5.2.1 (Wirthmüller isomorphism; see eg [36]) The map $w_{H}$ induces a natural isomorphism between the derived functors

$$
G \ltimes_{H}-: \operatorname{Ho}\left(\operatorname{Sp}_{H}^{O}\right) \longrightarrow \operatorname{Ho}\left(\operatorname{Sp}_{G}^{O}\right) \quad \text { and } \operatorname{Map}_{H}\left(G_{+},-\right): \operatorname{Ho}\left(\operatorname{Sp}_{H}^{O}\right) \longrightarrow \operatorname{Ho}\left(\operatorname{Sp}_{G}^{O}\right)
$$

That is, the left and right adjoint functors of

$$
\operatorname{Res}_{H}^{G}: \operatorname{Ho}\left(\operatorname{Sp}_{G}^{O}\right) \longrightarrow \operatorname{Ho}\left(\operatorname{Sp}_{H}^{O}\right)
$$

are naturally isomorphic.

As a consequence of the Wirthmüller isomorphism, one gets that for any subgroup $L \leq G$, the equivariant spectrum $\Sigma_{+}^{\infty} G / L$ is self-dual. Indeed, the map

$$
\Sigma_{+}^{\infty} G / L \cong G \ltimes_{L} \mathbb{S} \stackrel{w_{L}}{\longrightarrow} \operatorname{Map}_{L}\left(G_{+}, \mathbb{S}\right) \cong \operatorname{Map}\left(\Sigma_{+}^{\infty} G / L, \mathbb{S}\right) \cong D\left(\Sigma_{+}^{\infty} G / L\right)
$$

is an isomorphism in $\operatorname{Ho}\left(\mathrm{Sp}_{G}^{O}\right)$, where $D$ is the equivariant Spanier-Whitehead duality functor [30, Sections II.6, III.2, V.9; 35, Section XVI.7].

We conclude the subsection with the following well-known lemma and its corollaries. 
Lemma 5.2.2 (Double coset formula) Suppose $G$ is a finite group with arbitrary subgroups $H$ and $K$. For $g \in G$, let $c_{g}:{ }^{g} H \rightarrow H$ denote the map $c_{g}(x)=g^{-1} x g$. Then for any pointed $H$-set $X$, the $K$-equivariant maps

$$
K \ltimes g_{H \cap K} \operatorname{Res}_{g_{H} \cap K}^{g}\left(c_{g}^{*} X\right) \longrightarrow \operatorname{Res}_{K}^{G}\left(G \ltimes_{H} X\right), \quad[k, x] \mapsto[k g, x],
$$

induce a natural splitting

$$
\bigvee_{[g] \in K \backslash G / H} K \ltimes{ }{ }_{H \cap K} \operatorname{Res}_{g_{H}}^{g}{ }_{H}{ }\left(c_{g}^{*} X\right) \cong \operatorname{Res}_{K}^{G}\left(G \ltimes_{H} X\right)
$$

Corollary 5.2.3 Suppose $G$ is a finite group with subgroups $H$ and $K$. Then for any $Y \in \mathrm{Ho}\left(\mathrm{Sp}_{H}^{O}\right)$, there is a natural splitting

$$
\bigvee_{[g] \in K \backslash G / H} K \ltimes_{g_{H} \cap K} \operatorname{Res}_{g_{H} g_{H}}\left(c_{g}^{*} Y\right) \cong \operatorname{Res}_{K}^{G}\left(G \ltimes_{H} Y\right)
$$

Note that, if $X$ is a pointed $G$-set, then there is a natural isomorphism

$$
G \ltimes_{H} \operatorname{Res}_{H}^{G} X \cong G / H_{+} \wedge X
$$

given by $[g, x] \mapsto([g] \wedge g x)$.

Corollary 5.2.4 The maps

$$
G /\left({ }^{g} H \cap K\right)_{+} \longrightarrow G / H_{+} \wedge G / K_{+}, \quad[x] \mapsto[x g] \wedge[x],
$$

of pointed $G$-sets induce a natural splitting

$$
\bigvee_{[g] \in K \backslash G / H} G /\left({ }^{g} H \cap K\right)_{+} \stackrel{\cong}{\longrightarrow} G / H_{+} \wedge G / K_{+} .
$$

Proof By the last observation and Lemma 5.2.2, we have a chain of isomorphisms of pointed $G$-sets:

$$
\begin{aligned}
\bigvee_{[g] \in K \backslash G / H} G /\left({ }^{g} H \cap K\right)_{+} & \cong \bigvee_{[g] \in K \backslash G / H} G \ltimes_{K}\left(K /\left({ }^{g} H \cap K\right)\right)_{+} \\
& \cong G \ltimes_{K}\left(\bigvee_{[g] \in K \backslash G / H} K /\left({ }^{g} H \cap K\right)_{+}\right) \\
& \cong G \ltimes_{K}\left(\bigvee_{[g] \in K \backslash G / H} K \ltimes^{g} H \cap K\right. \\
& \left.S^{0}\right) \\
& \cong G \ltimes_{K} \operatorname{Res}_{K}^{G}\left(G \ltimes_{H} S^{0}\right) \cong G \ltimes_{K} \operatorname{Res}_{K}^{G}\left(G / H_{+}\right) \\
& \cong G / K_{+} \wedge G / H_{+} \cong G / H_{+} \wedge G / K_{+} .
\end{aligned}
$$


Here the last isomorphism is the twist. Going through these explicit isomorphisms, we see that any $[x] \in G /\left({ }^{g} H \cap K\right)_{+}$is sent to $[x g] \wedge[x] \in G / H_{+} \wedge G / K_{+}$.

\subsection{Proof of Proposition 5.1.1}

As we already mentioned after Proposition 5.2.1, we have the isomorphisms

$$
D\left(\Sigma_{+}^{\infty} G / L\right) \cong \Sigma_{+}^{\infty} G / L, \quad L \leq G,
$$

in $\operatorname{Ho}\left(\operatorname{Sp}_{G}^{O}\right)$, where $D$ is the equivariant Spanier-Whitehead duality. It follows from [30, Sections II.6, III.2, V.9] (see also [29]) that, under these isomorphisms, the transfer maps correspond to restrictions. In particular, for any $g \in G$, the diagram

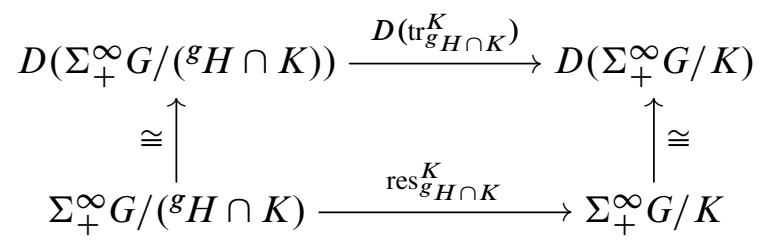

commutes. Combining this with the Spanier-Whitehead duality, for any $g \in G$, one gets the following commutative diagram with all vertical maps isomorphisms:

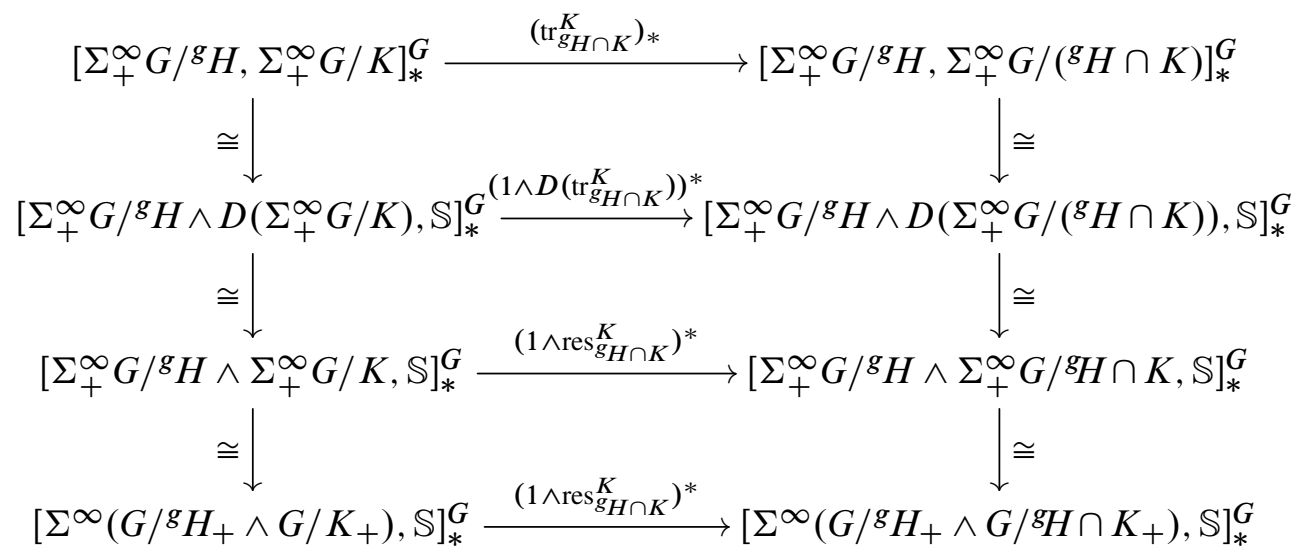

Again using the Spanier-Whitehead duality and that $\Sigma_{+}^{\infty} G / L$ is self-dual for $L \leq G$, we also have that, for every $g \in G$, the diagrams 


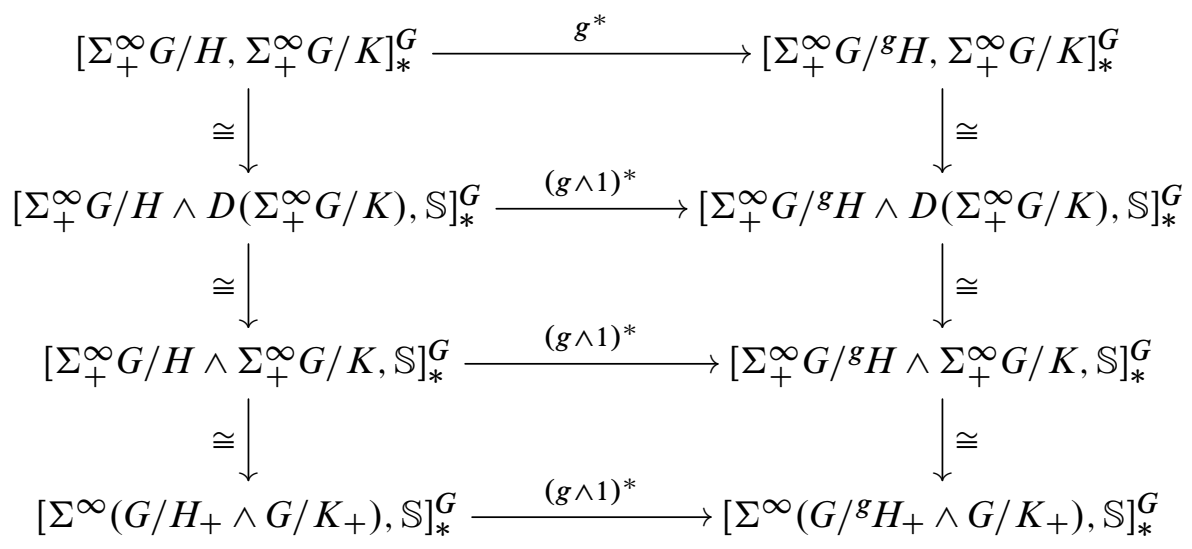

and

$\left[\Sigma_{+}^{\infty} G /{ }^{g} H, \Sigma_{+}^{\infty} G /{ }^{g} H \cap K\right]_{*}^{G} \stackrel{\left(\operatorname{res}_{g_{H}}^{g_{H}}\right)^{*}}{\longrightarrow}\left[\Sigma_{+}^{\infty} G /{ }^{g} H \cap K, \Sigma_{+}^{\infty} G /{ }^{g} H \cap K\right]_{*}^{G}$

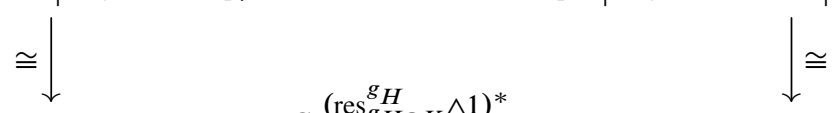

$\left[\Sigma_{+}^{\infty} G /{ }^{g} H \wedge D\left(\Sigma_{+}^{\infty} G /{ }^{g} H \cap K\right), \mathbb{S}\right]_{*}^{G} \stackrel{\left.\text { (res }_{g_{H}}^{g_{H}}{ }^{\wedge 1}\right)^{*}}{\longrightarrow}\left[\Sigma_{+}^{\infty} G /{ }^{g} H \cap K \wedge D\left(\Sigma_{+}^{\infty} G /{ }^{g} H \cap K\right), \mathbb{S}\right]_{*}^{G}$

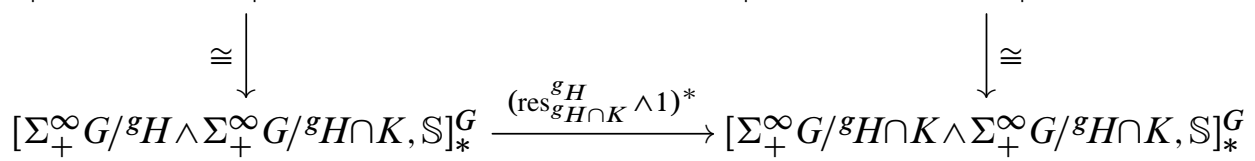

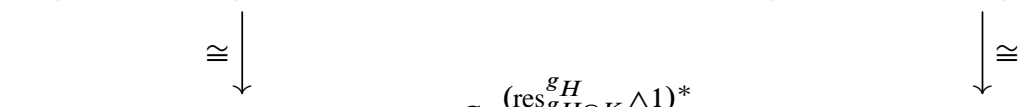

$\left[\Sigma^{\infty}\left(G /{ }^{g} H_{+} \wedge G /{ }^{g} H \cap K_{+}\right), \mathbb{S}\right]_{*}^{G} \stackrel{\left.\operatorname{res}_{g_{H}}^{g_{H}} \wedge\right)^{*}}{\longrightarrow}\left[\Sigma^{\infty}\left(G /{ }^{g} H \cap K_{+} \wedge G /{ }^{g} H \cap K_{+}\right), \mathbb{S}\right]_{*}^{G}$

commute. Hence by definition, for any $g \in G$, the morphism

$$
\kappa_{g}:\left[\Sigma_{+}^{\infty} G / H, \Sigma_{+}^{\infty} G / K\right]_{*}^{G} \longrightarrow\left[\Sigma_{+}^{\infty} G /\left({ }^{g} H \cap K\right), \Sigma_{+}^{\infty} G /\left({ }^{g} H \cap K\right)\right]_{*}^{G}
$$

is isomorphic to the morphism induced by the composite

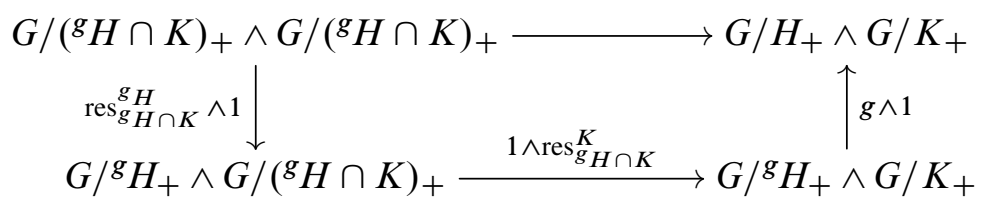

after applying the functor $\left[\Sigma^{\infty}(-), \mathbb{S}\right]_{*}^{G}$. To simplify notations let us denote this composite of maps of pointed $G$-sets by $\theta_{g}: G /\left({ }^{g} H \cap K\right)_{+} \wedge G /\left({ }^{g} H \cap K\right)_{+} \rightarrow$ $G / H_{+} \wedge G / K_{+}$. Thus, in order to prove Proposition 5.1.1, it suffices to check that the 
map of pointed $G$-sets

$$
\bigvee_{[g] \in K \backslash G / H}\left(G /\left({ }^{g} H \cap K\right)_{+} \wedge G /\left({ }^{g} H \cap K\right)_{+}\right) \stackrel{\left(\theta_{g}\right)_{[g] \in K \backslash G / H}}{\longrightarrow} G / H_{+} \wedge G / K_{+}
$$

has a $G$-equivariant section. This follows from the commutative diagram of pointed $G$-sets

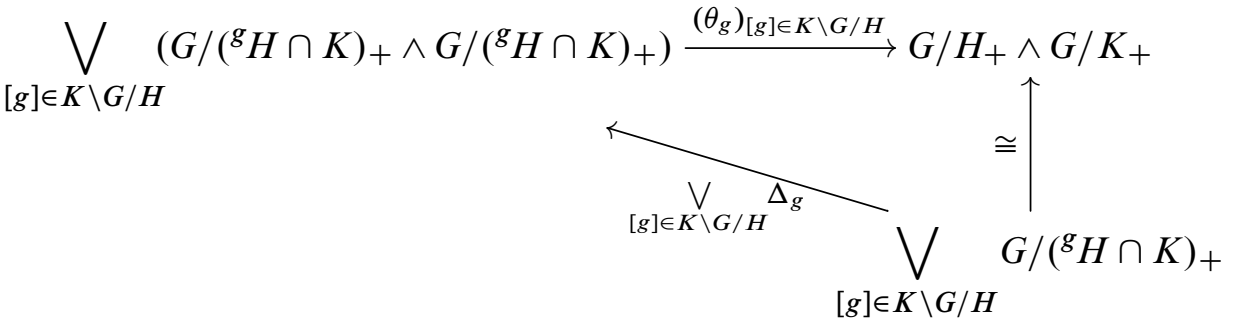

where the vertical map is the isomorphism from Corollary 5.2.4, and

$$
\Delta_{g}: G /\left({ }^{g} H \cap K\right)_{+} \longrightarrow G /\left({ }^{g} H \cap K\right)_{+} \wedge G /\left({ }^{g} H \cap K\right)_{+}
$$

is the diagonal defined by $[x] \mapsto[x] \wedge[x]$ for any $g$.

\section{A short exact sequence}

This section constructs a split short exact sequence that will play a fundamental role in the inductive proof of Theorem 3.1.3. The author thinks that this short exact sequence is well-known to the experts. However, since we were unable to find a reference, we decided to provide a detailed proof here.

\subsection{Geometric fixed points and the inflation functor}

To construct the desired short exact sequence, we need the geometric fixed point functor

$$
\Phi^{N}: \mathrm{Sp}_{G}^{O} \longrightarrow \mathrm{Sp}_{J}^{O}
$$

associated to an extension of finite groups

$$
E: 1 \longrightarrow N \stackrel{\iota}{\longrightarrow} G \stackrel{\varepsilon}{\longrightarrow} J \longrightarrow 1 .
$$

This functor is constructed in [33, Section V.4] and has some useful properties. In particular, the following holds: 
Proposition 6.1.1 [33, Proposition V.4.5] Let $A$ be a pointed $G$-space and $V$ a finite-dimensional orthogonal $G$-representation. Then there is a natural isomorphism of $J$-spectra

$$
\Phi^{N}\left(F_{V} A\right) \cong F_{V^{N}} A^{N}
$$

Also, the functor $\Phi^{N}: \mathrm{Sp}_{G}^{O} \longrightarrow \mathrm{Sp}_{J}^{O}$ preserves cofibrations and acyclic cofibrations.

Corollary 6.1.2 For any based $G$-space $A$, there is a natural isomorphism of $J_{-}$ spectra

$$
\Phi^{N}\left(\Sigma^{\infty} A\right) \cong \Sigma^{\infty}\left(A^{N}\right) \text {. }
$$

Next, we will also need the inflation (change of scalars) functor $\varepsilon^{*}: \operatorname{Sp}_{J}^{O} \rightarrow \operatorname{Sp}_{G}^{O}$ associated to an extension of finite groups

$$
E: 1 \longrightarrow N \stackrel{\iota}{\longrightarrow} G \stackrel{\varepsilon}{\longrightarrow} J \longrightarrow 1 .
$$

This functor is a left adjoint to the point-set level (categorical) fixed point functor. In fact, it is a left Quillen functor.

By Proposition 6.1.1 and Ken Brown's lemma, one can derive the functor $\Phi^{N}$ and get the functor

$$
\Phi^{N}: \mathrm{Ho}\left(\mathrm{Sp}_{G}^{O}\right) \longrightarrow \mathrm{Ho}\left(\mathrm{Sp}_{J}^{O}\right) .
$$

We can also derive the left Quillen functor $\varepsilon^{*}$ and obtain the derived inflation

$$
\varepsilon^{*}: \operatorname{Ho}\left(\operatorname{Sp}_{J}^{O}\right) \longrightarrow \operatorname{Ho}\left(\operatorname{Sp}_{G}^{O}\right) .
$$

The following result follows from [30, Proposition II.9.10] and [33, Sections VI.3-5].

Proposition 6.1.3 There is a triangulated natural isomorphism

$$
\nu: \mathrm{id} \stackrel{\cong}{\longrightarrow} \Phi^{N} \varepsilon^{*}
$$

of endofunctors on $\mathrm{Ho}\left(\mathrm{Sp}_{J}^{O}\right)$.

\subsection{Weyl groups}

Let $G$ be a finite group and $H$ a subgroup of $G$. Then $H$ is a normal subgroup of its normalizer $N(H)=\{g \in G \mid g H=H g\}$, and the quotient group $W(H)=$ $N(H) / H$ is called the Weyl group of $H$. According to the previous subsection, the short exact sequence

$$
1 \longrightarrow H \stackrel{\iota}{\longrightarrow} N(H) \stackrel{\varepsilon}{\longrightarrow} W(H) \longrightarrow 1
$$


gives us the geometric fixed point functor

$$
\Phi^{H}: \operatorname{Ho}\left(\operatorname{Sp}_{N(H)}^{O}\right) \longrightarrow \operatorname{Ho}\left(\operatorname{Sp}_{W(H)}^{O}\right)
$$

and the inflation functor

$$
\varepsilon^{*}: \operatorname{Ho}\left(\mathrm{Sp}_{W(H)}^{O}\right) \longrightarrow \operatorname{Ho}\left(\mathrm{Sp}_{N(H)}^{O}\right) .
$$

We will denote the composite functor

$$
\Phi^{H} \circ \operatorname{Res}_{N(H)}^{G}: \operatorname{Ho}\left(\operatorname{Sp}_{G}^{O}\right) \longrightarrow \operatorname{Ho}\left(\operatorname{Sp}_{W(H)}^{O}\right)
$$

by $\Phi_{\text {res }}^{H}$. It then follows from Corollary 6.1.2 that there is an isomorphism

$$
\Phi_{\text {res }}^{H}\left(\Sigma_{+}^{\infty} G / H\right) \cong \Sigma_{+}^{\infty}(G / H)^{H}
$$

in $\operatorname{Ho}\left(\mathrm{Sp}_{W(H)}^{O}\right)$. (This holds already on the point-set level.) Since $(G / H)^{H}=W(H)$ as $W(H)$-sets, one gets an isomorphism

$$
\Phi_{\text {res }}^{H}\left(\Sigma_{+}^{\infty} G / H\right) \cong \Sigma_{+}^{\infty} W(H)
$$

in $\operatorname{Ho}\left(\operatorname{Sp}_{W(H)}^{O}\right)$. Further, by definition, one has $\varepsilon^{*}\left(\Sigma_{+}^{\infty} W(H)\right)=\Sigma_{+}^{\infty} N(H) / H$ in $\mathrm{Ho}\left(\mathrm{Sp}_{N(H)}^{O}\right)$, and hence we get

$$
G \ltimes_{N(H)} \varepsilon^{*}\left(\Sigma_{+}^{\infty} W(H)\right)=\Sigma_{+}^{\infty} G / H .
$$

Having these identifications in mind, we are now ready to formulate the following:

Proposition 6.2.1 Let $W$ denote the Weyl group, $W(H)$. The composite

$$
\left[\Sigma_{+}^{\infty} W, \Sigma_{+}^{\infty} W\right]_{*}^{W} \stackrel{G \ltimes_{N(H)} \varepsilon^{*}}{\longrightarrow}\left[\Sigma_{+}^{\infty} G / H, \Sigma_{+}^{\infty} G / H\right]_{*}^{G} \stackrel{\Phi_{\mathrm{res}}^{H}}{\longrightarrow}\left[\Sigma_{+}^{\infty} W, \Sigma_{+}^{\infty} W\right]_{*}^{W}
$$

is an isomorphism.

Proof By Proposition 6.1.3, we have the natural isomorphism

$$
\nu: \mathrm{id} \stackrel{\cong}{\longrightarrow} \Phi^{H} \varepsilon^{*},
$$

which is a triangulated transformation. Since $\left(G \ltimes_{N(H)}-, \operatorname{Res}_{N(H)}^{G}\right)$ is a Quillen adjunction (see Subsection 5.2), the derived unit map

$$
\mathrm{id} \stackrel{\eta}{\longrightarrow} \operatorname{Res}_{N(H)}^{G}\left(G \ltimes_{N(H)}-\right)
$$

is a triangulated transformation as well; see Subsection 2.2. Hence, the composite $\Phi^{H} \eta \varepsilon^{*} \circ \nu:$ id $\stackrel{v}{\longrightarrow} \Phi^{H} \varepsilon^{*} \stackrel{\Phi^{H} \eta \varepsilon^{*}}{\longrightarrow} \Phi^{H}\left(\operatorname{Res}_{N(H)}^{G}\left(G \ltimes_{N(H)} \varepsilon^{*}\right)\right)=\Phi_{\text {res }}^{H}\left(G \ltimes_{N(H)} \varepsilon^{*}\right)$ 
is a triangulated transformation. Moreover, it follows from Proposition 6.1.3 and the identifications we did before Proposition 6.2.1 that this natural transformation is an isomorphism in $\operatorname{Ho}\left(\operatorname{Sp}_{W(H)}^{O}\right)$ when applied to $\Sigma_{+}^{\infty} W(H)$. Consequently, by Lemma 2.2.1, the restriction of the functor $\Phi^{H}\left(\operatorname{Res}_{N(H)}^{G}\left(G \ltimes_{N(H)} \varepsilon^{*}\right)\right)$ to the localizing subcategory of $\mathrm{Ho}\left(\mathrm{Sp}_{W(H)}^{O}\right)$ generated by $\Sigma_{+}^{\infty} W(H)$ is isomorphic to the identity functor and thus an equivalence of categories. This implies the desired result.

\subsection{The short exact sequence}

Suppose $G$ is a finite group and $\mathscr{F}$ a set of subgroups of $G$. The set $\mathscr{F}$ is said to be a family of subgroups of $G$ if it is closed under conjugation and taking subgroups.

Recall that, for any finite group $G$ and any family $\mathscr{F}$, there is a classifying space ( $G$-CW complex) $E \mathscr{F}$ characterized up to $G$-homotopy equivalence by the property that $E \mathscr{F}^{H}$ is contractible if $H \in \mathscr{F}$ and $E \mathscr{F}^{H}=\varnothing$ if $H \notin \mathscr{F}$; see eg [14].

Let $\mathscr{P}$ denote the family of proper subgroups of $G$. Consider the equivariant map $E \mathscr{P}_{+} \stackrel{\text { proj }}{\longrightarrow} S^{0}$ which sends the elements of $E \mathscr{P}$ to the nonbase point of $S^{0}$. The mapping cone sequence of this map (called the isotropy separation sequence) combined with the tom Dieck splitting [10, Theorem II.7.7] gives the following well-known fact:

Proposition 6.3.1 Let $G$ be a finite group. Then there is a split short exact sequence

$$
0 \longrightarrow\left[S, \Sigma_{+}^{\infty} E \mathscr{P}\right]_{*}^{G} \stackrel{\operatorname{proj}_{*}}{\longrightarrow}[\mathbb{S}, \mathbb{S}]_{*}^{G} \stackrel{\Phi^{G}}{\longrightarrow}[\mathbb{S}, \mathbb{S}]_{*} \longrightarrow 0 .
$$

Now suppose $H$ is a subgroup of $G$. Then for any $X \in \operatorname{Ho}\left(\operatorname{Sp}_{G}^{O}\right)$, there is a natural isomorphism

$$
G \ltimes_{H} \operatorname{Res}_{H}^{G} X \cong G / H_{+} \wedge^{\mathbf{L}} X
$$

given on the point-set level by $[g, x] \mapsto([g] \wedge g x)$. In particular,

$$
G \ltimes_{H} \mathbb{S} \cong \Sigma_{+}^{\infty} G / H .
$$

Having this preferred isomorphism in mind, we will once and for all identify $G \ltimes_{H} \mathbb{S}$ with $\Sigma_{+}^{\infty} G / H$. Next, let $\mathscr{P}[H]$ denote the family of proper subgroups of $H$. Note that this is a family with respect to $H$ and not necessarily with respect to the whole group $G$. Here is the main result of this section, which is an important tool in the proof of Theorem 3.1.3.

Proposition 6.3.2 Let $G$ be a finite group, $H$ a subgroup, and $W$ the Weyl group, $W(H)$. Then there is a split short exact sequence $\left[\Sigma_{+}^{\infty} G / H, G \ltimes_{H} \Sigma_{+}^{\infty} E \mathscr{P}[H]\right]_{*}^{G} \stackrel{\operatorname{proj}_{*}}{\longrightarrow}\left[\Sigma_{+}^{\infty} G / H, \Sigma_{+}^{\infty} G / H\right]_{*}^{G} \stackrel{\Phi_{\text {res }}^{H}}{\longrightarrow}\left[\Sigma_{+}^{\infty} W, \Sigma_{+}^{\infty} W\right]_{*}^{W}$, 
where the morphism proj: $G \ltimes_{H} \Sigma_{+}^{\infty} E \mathscr{P}[H] \longrightarrow \Sigma_{+}^{\infty} G / H$ is defined as the composite

$$
G \ltimes_{H} \Sigma_{+}^{\infty} E \mathscr{P}[H] \stackrel{G \ltimes_{H} \operatorname{proj}}{\longrightarrow} G \ltimes_{H} \mathbb{S} \cong \Sigma_{+}^{\infty} G / H .
$$

Before proving this proposition we have to recall some important technical facts.

\subsection{Technical preparation}

It follows from the definition that, for any $K \in \mathscr{P}[H]$, the set of $H$-fixed points of $G / K$ is empty. Together with Corollary 6.1.2, this implies that $\Phi_{\text {res }}^{H}\left(\Sigma_{+}^{\infty} G / K\right)=*$ in $\operatorname{Ho}\left(\mathrm{Sp}_{W(H)}^{O}\right)$. Since the classifying space $E \mathscr{P}[H]$ is built out of $H$-cells of orbit type $H / K$ with $K \leq H$ and $K \neq H$, one obtains:

Proposition 6.4.1 Let $G$ be a finite group. For any subgroup $H \leq G$, the $G-C W$ complex $G \times{ }_{H} E \mathscr{P}[H]$ is built out of $G$-cells of orbit type $G / K$ with $K \leq H$ and $K \neq H$. Furthermore, $\Phi_{\text {res }}^{H}\left(G \ltimes_{H} \Sigma_{+}^{\infty} E \mathscr{P}[H]\right)=*$ in $\operatorname{Ho}\left(\operatorname{Sp}_{W(H)}^{O}\right)$.

Next, we recall that the functor $\operatorname{Map}_{H}\left(G_{+},-\right): \operatorname{Ho}\left(\operatorname{Sp}_{H}^{O}\right) \rightarrow \operatorname{Ho}\left(\operatorname{Sp}_{G}^{O}\right)$ is right adjoint to $\operatorname{Res}_{H}^{G}$. Recall also the map $w_{H}: G \ltimes_{H}(-) \rightarrow \operatorname{Map}_{H}\left(G_{+},-\right)$that induces the Wirthmüller isomorphism; see Proposition 5.2.1. The following proposition is a consequence of the Wirthmüller isomorphism.

Proposition 6.4.2 For any $Y$ in $\operatorname{Ho}\left(\operatorname{Sp}_{H}^{O}\right)$, the natural map

$$
\left[\mathbb{S}, G \ltimes_{H} Y\right]_{*}^{G} \stackrel{w_{H *}}{\longrightarrow}\left[\mathbb{S}, \operatorname{Map}_{H}\left(G_{+}, Y\right)\right]_{*}^{G} \cong\left[\operatorname{Res}_{H}^{G}(\mathbb{S}), Y\right]_{*}^{H}=[\mathbb{S}, Y]_{*}^{H}
$$

is an isomorphism.

Corollary 6.4.3 Let $G$ be a finite group with subgroups $H$ and $K$. Then for any spectrum $Y \in \operatorname{Ho}\left(\mathrm{Sp}_{H}^{O}\right)$, there is a natural isomorphism

$$
\left[\Sigma_{+}^{\infty} G / K, G \ltimes_{H} Y\right]_{*}^{G} \cong \bigoplus_{[g] \in K \backslash G / H}\left[S, \operatorname{Res}_{K \cap^{g} H_{H}}^{g_{H}}\left(c_{g}^{*}(Y)\right)\right]_{*}^{K \cap{ }^{g} H}
$$


Proof By adjunction, Corollary 5.2.3 and Proposition 6.4.2, one has the following chain of isomorphisms:

$$
\begin{aligned}
{\left[\Sigma_{+}^{\infty} G / K, G \ltimes_{H} Y\right]_{*}^{G} } & \cong\left[G \ltimes_{K} \mathbb{S}, G \ltimes_{H} Y\right]_{*}^{G} \cong\left[\mathbb{S}, \operatorname{Res}_{K}^{G}\left(G \ltimes_{H} Y\right)\right]_{*}^{K} \\
& \cong\left[\mathbb{S}, \bigvee_{[g] \in K \backslash G / H} K \ltimes_{K \cap{ }^{g} H} \operatorname{Res}_{K \cap{ }^{g}{ }_{H}}\left(c_{g}^{*}(Y)\right)\right]_{*}^{K} \\
& \cong \bigoplus_{[g] \in K \backslash G / H}\left[\mathbb{S}, K \ltimes_{K \cap{ }^{g} H} \operatorname{Res}_{K \cap{ }^{g}{ }_{H}}^{g}\left(c_{g}^{*}(Y)\right)\right]_{*}^{K} \\
& \cong \bigoplus_{[g] \in K \backslash G / H}\left[\mathbb{S}, \operatorname{Res}_{K{ }^{g}{ }^{g} H}^{g}\left(c_{g}^{*}(Y)\right)\right]_{*}^{K \cap{ }^{g} H} .
\end{aligned}
$$

\subsection{Proof of Proposition 6.3.2}

In this subsection we prove that the sequence $\left[\Sigma_{+}^{\infty} G / H, G \ltimes_{H} \Sigma_{+}^{\infty} E \mathscr{P}[H]\right]_{*}^{G} \stackrel{\operatorname{proj}_{*}}{\longrightarrow}\left[\Sigma_{+}^{\infty} G / H, \Sigma_{+}^{\infty} G / H\right]_{*}^{G} \stackrel{\Phi_{\mathrm{res}}^{H}}{\longrightarrow}\left[\Sigma_{+}^{\infty} W, \Sigma_{+}^{\infty} W\right]_{*}^{W}$ is a split short exact sequence. It follows from Proposition 6.2.1 that

$$
\Phi_{\text {res }}^{H}:\left[\Sigma_{+}^{\infty} G / H, \Sigma_{+}^{\infty} G / H\right]_{*}^{G} \longrightarrow\left[\Sigma_{+}^{\infty} W, \Sigma_{+}^{\infty} W\right]_{*}^{W}
$$

is a retraction and thus, in particular, surjective. Further, Proposition 6.4.1 implies that

$$
\Phi_{\text {res }}^{H} \circ \operatorname{proj}_{*}=0 \text {. }
$$

Hence, it remains to show that the map

$$
\operatorname{proj}_{*}:\left[\Sigma_{+}^{\infty} G / H, G \ltimes_{H} \Sigma_{+}^{\infty} E \mathscr{P}[H]\right]_{*}^{G} \longrightarrow\left[\Sigma_{+}^{\infty} G / H, \Sigma_{+}^{\infty} G / H\right]_{*}^{G}
$$

is injective and $\operatorname{Ker} \Phi_{\text {res }}^{H} \subset \operatorname{Im}\left(\operatorname{proj}_{*}\right)$. For this we choose a set $\{g\}$ of double coset representatives for $H \backslash G / H$. By Corollary 6.4.3, the following is a commutative diagram with all vertical arrows isomorphisms:

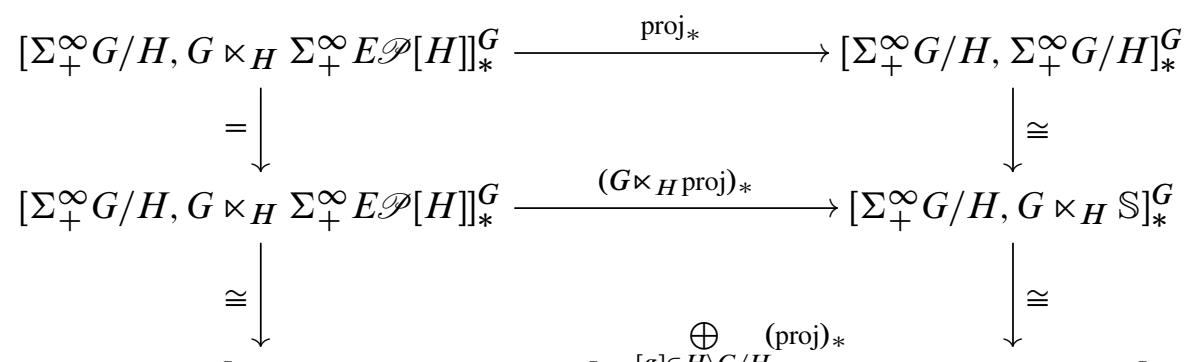

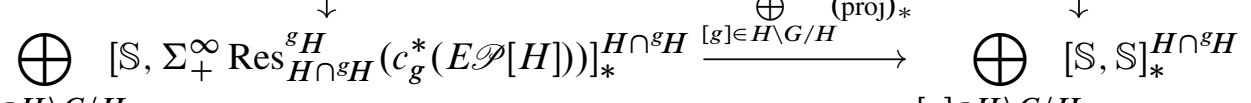
$[g] \in H \backslash G / H$ 
We will now identify the summands of the lower horizontal map. For this, one has to consider two cases.

Case $1 \quad H \cap{ }^{g} H=H$ : In this case, $\operatorname{Res}_{H \cap{ }^{g}{ }_{H}}\left(c_{g}^{*}(E \mathscr{P}[H])\right)=c_{g}^{*}(E \mathscr{P}[H])$ is a model for the classifying space of $\mathscr{P}[H]$ (and hence $G$-homotopy equivalent to $E \mathscr{P}[H])$ ). By Proposition 6.3.1, we get a short exact sequence $0 \rightarrow\left[S, \Sigma_{+}^{\infty} \operatorname{Res}_{H \cap g_{H}^{g}}^{g_{H}}\left(c_{g}^{*}(E \mathscr{P}[H])\right)\right]_{*}^{H \cap{ }^{g} H} \stackrel{\operatorname{proj}_{*}}{\longrightarrow}[\mathbb{S}, \mathbb{S}]_{*}^{H \cap \cap^{g} H} \stackrel{\Phi^{H} \cap^{g} H}{\longrightarrow}[\mathbb{S}, \mathbb{S}]_{*} \rightarrow 0$.

Case $2 H \cap{ }^{g} H$ is a proper subgroup of $H$ : In this case, $\operatorname{Res}_{H \cap{ }^{g}{ }_{H}}\left(c_{g}^{*}(E \mathscr{P}[H])\right)$ is an $\left(H \cap{ }^{g} H\right)$-contractible cofibrant $\left(H \cap{ }^{g} H\right)$-space and hence the map

$$
\left[\mathbb{S}, \Sigma_{+}^{\infty} \operatorname{Res}_{H \cap{ }^{g} H}^{g_{H}}\left(c_{g}^{*}(E \mathscr{P}[H])\right)\right]_{*}^{H \cap{ }^{g} H} \stackrel{\text { proj }_{*}}{\longrightarrow}[S, S]_{*}^{H \cap{ }^{g} H}
$$

is an isomorphism.

After combining the latter diagram with case 1 and case 2, we see that the map

$$
\operatorname{proj}_{*}:\left[\Sigma_{+}^{\infty} G / H, G \ltimes_{H} \Sigma_{+}^{\infty} E \mathscr{P}[H]\right]_{*}^{G} \longrightarrow\left[\Sigma_{+}^{\infty} G / H, \Sigma_{+}^{\infty} G / H\right]_{*}^{G}
$$

is injective. It still remains to check that $\operatorname{Ker} \Phi_{\text {res }}^{H} \subset \operatorname{Im}\left(\operatorname{proj}_{*}\right)$. For this, first note that $H \cap{ }^{g} H=H$ if and only if $g \in N(H)$. Further, if $g \in N(H)$, then the double coset class $H g H$ is equal to $g H$. Hence the set of those double cosets $[g] \in H \backslash G / H$ for which the equality $H \cap{ }^{g} H=H$ holds is in bijection with the Weyl group $W$. Consequently, using the latter diagram, case 1 (and hence Proposition 6.3.1) and case 2 , one gets an isomorphism

$$
\left[\Sigma_{+}^{\infty} G / H, \Sigma_{+}^{\infty} G / H\right]_{*}^{G} / \operatorname{Im}\left(\operatorname{proj}_{*}\right) \cong \bigoplus_{W}[\mathbb{S}, \mathbb{S}]_{*} \cong\left[\Sigma_{+}^{\infty} W, \Sigma_{+}^{\infty} W\right]_{*}^{W} .
$$

On the other hand, we have already checked that

$$
\Phi_{\text {res }}^{H}:\left[\Sigma_{+}^{\infty} G / H, \Sigma_{+}^{\infty} G / H\right]_{*}^{G} \longrightarrow\left[\Sigma_{+}^{\infty} W, \Sigma_{+}^{\infty} W\right]_{*}^{W}
$$

is surjective and this yields an isomorphism

$$
\left[\Sigma_{+}^{\infty} G / H, \Sigma_{+}^{\infty} G / H\right]_{*}^{G} / \operatorname{Ker} \Phi_{\text {res }}^{H} \cong\left[\Sigma_{+}^{\infty} W, \Sigma_{+}^{\infty} W\right]_{*}^{W} .
$$

Combining this with the previous isomorphism implies that the graded abelian group

$$
\left[\Sigma_{+}^{\infty} G / H, \Sigma_{+}^{\infty} G / H\right]_{*}^{G} / \operatorname{Im}\left(\operatorname{proj}_{*}\right)
$$

is isomorphic to

$$
\left[\Sigma_{+}^{\infty} G / H, \Sigma_{+}^{\infty} G / H\right]_{*}^{G} / \operatorname{Ker} \Phi_{\text {res }}^{H} .
$$

Now if the grading $*>0$, then $\left[\Sigma_{+}^{\infty} G / H, \Sigma_{+}^{\infty} G / H\right]_{*}^{G}$ is finite, and it follows that $\operatorname{Im}\left(\operatorname{proj}_{*}\right)$ and $\operatorname{Ker} \Phi_{\text {res }}^{H}$ are finite groups of the same cardinality; see Subsection 2.8. 
Since we already know that $\operatorname{Im}\left(\operatorname{proj}_{*}\right) \subset \operatorname{Ker} \Phi_{\text {res }}^{H}$ (we have already observed that this is a consequence of Proposition 6.4.1.), one finally gets the equality $\operatorname{Im}\left(\operatorname{proj}_{*}\right)=\operatorname{Ker} \Phi_{\text {res }}^{H}$. For $*=0$, a five lemma argument completes the proof. We do not give here the details of the case $*=0$ as it is irrelevant for our proof of Theorem 3.1.3.

\section{Proof of the main theorem}

In this section we complete the proof of Theorem 3.1.3 and hence of Theorem 1.1.1. We will start by recalling from [33, Section IV.6] the $\mathscr{F}$-model structure on the category of $G$-equivariant orthogonal spectra, where $\mathscr{F}$ is a family of subgroups of a finite group $G$.

\subsection{The $\mathscr{F}$-model structure and localizing subcategory determined by $\mathscr{F}$}

Let $G$ be a finite group and $\mathscr{F}$ a family of subgroups of $G$.

Definition 7.1.1 A morphism $f: X \rightarrow Y$ of $G$-equivariant orthogonal spectra is called an $\mathscr{F}$-equivalence if it induces isomorphisms

$$
f_{*}: \pi_{*}^{H} X \stackrel{\cong}{\longrightarrow} \pi_{*}^{H} Y
$$

on $H$-equivariant homotopy groups for any $H \in \mathscr{F}$. Similarly, a morphism $g: X \rightarrow Y$ in $\mathrm{Ho}\left(\mathrm{Sp}_{G}^{O}\right)$ is called an $\mathscr{F}$-equivalence if it induces an isomorphism on $\pi_{*}^{H}$ for any $H \in \mathscr{F}$.

The category of $G$-equivariant orthogonal spectra has a stable model structure with weak equivalences the $\mathscr{F}$-equivalences and with cofibrations the $\mathscr{F}$-cofibrations; see [33, Theorem IV.6.5]. By restricting our attention to those orbits $G / H$ that satisfy $H \in \mathscr{F}$, we can obtain the generating $\mathscr{F}$-cofibrations and acyclic $\mathscr{F}$-cofibrations in a similar way as for the absolute case of $\operatorname{Sp}_{G}^{O}$ [33, Section III.4]; see Sections 2.6 and 2.7. In particular, retracts of relative $\mathscr{F}$-cell complexes built out of generating $\mathscr{F}$-cofibrations are exactly $\mathscr{F}$-cofibrations. We will denote this model category by $\operatorname{Sp}_{G, \mathscr{F}}^{O}$.

The following proposition relates the classifying space $E \mathscr{F}$ with the concept of an $\mathscr{F}$-equivalence.

Proposition 7.1.2 [33, Proposition IV.6.7] A morphism $f: X \rightarrow Y$ of $G$-equivariant orthogonal spectra is an $\mathscr{F}$-equivalence if and only if $1 \wedge f: E \mathscr{F}+\wedge X \rightarrow E \mathscr{F}+\wedge Y$ is a $G$-equivalence, ie a stable equivalence of orthogonal $G$-spectra. 
By definition of $\mathscr{F}$-equivalences and $\mathscr{F}$-cofibrations, we get a Quillen adjunction

$$
\text { id: } \mathrm{Sp}_{G, \mathscr{F}}^{O} \rightleftarrows \mathrm{Sp}_{G}^{O}: \mathrm{id} \text {. }
$$

After deriving this Quillen adjunction one obtains an adjunction

$$
\mathbf{L}: \operatorname{Ho}\left(\operatorname{Sp}_{G, \mathscr{F}}^{O}\right) \rightleftarrows \mathrm{Ho}\left(\operatorname{Sp}_{G}^{O}\right): \mathbf{R}
$$

on the homotopy level. We now examine the essential image of the left adjoint functor L. Since a weak equivalence in $\operatorname{Sp}_{G}^{O}$ is also a weak equivalence in $\operatorname{Sp}_{G, \mathscr{F}}^{O}$, the unit

$$
\text { id } \longrightarrow \text { RL }
$$

of the adjunction $(\mathbf{L}, \mathbf{R})$ is an isomorphism of functors. Hence the functor

$$
\mathbf{L}: \operatorname{Ho}\left(\operatorname{Sp}_{G, \mathscr{F}}^{O}\right) \longrightarrow \operatorname{Ho}\left(\operatorname{Sp}_{G}^{O}\right)
$$

is fully faithful.

Proposition 7.1.3 For any $X \in \mathrm{Sp}_{G, \mathscr{F}}^{O}$, there are natural isomorphisms

$$
\mathbf{L}(X) \cong E \mathscr{F}_{+} \wedge^{\mathbf{L}} X \cong E \mathscr{F}+\wedge X .
$$

Proof Let $\lambda_{X}: X^{c} \rightarrow X$ be a (functorial) cofibrant replacement of $X$ in $\operatorname{Sp}_{G, \mathscr{F}}^{O}$. By [33, Theorem IV.6.10], we have that the projection map $E \mathscr{F}+\wedge X^{c} \rightarrow X^{c}$ is a weak equivalence in $\mathrm{Sp}_{G}^{O}$. On the other hand, Proposition 7.1.2 implies that the morphism of $G$-spectra $1 \wedge \lambda_{X}: E \mathscr{F}_{+} \wedge X^{c} \rightarrow E \mathscr{F}_{+} \wedge X$ is a weak equivalence in $\operatorname{Sp}_{G}^{O}$. This completes the proof.

Next, note that the triangulated category $\operatorname{Ho}\left(\operatorname{Sp}_{G, \mathscr{F}}^{O}\right)$ is compactly generated with

$$
\left\{\Sigma_{+}^{\infty} G / H \mid H \in \mathscr{F}\right\}
$$

see Subsection 2.2. Indeed, this follows from the following chain of isomorphisms:

$$
\begin{aligned}
& {\left[\Sigma_{+}^{\infty} G / H, X\right]_{*}^{\mathrm{Ho}\left(\mathrm{Sp}_{G, \mathscr{F}}^{O}\right)} \cong\left[E \mathscr{F}+\wedge \Sigma_{+}^{\infty} G / H, E \mathscr{F}+\wedge X\right]_{*}^{G} } \\
& \cong\left[\Sigma_{+}^{\infty} G / H, E \mathscr{F}_{+} \wedge X\right]_{*}^{G} \cong \pi_{*}^{H}\left(E \mathscr{F}_{+} \wedge X\right) \cong \pi_{*}^{H} X .
\end{aligned}
$$

The first isomorphism in this chain follows from Proposition 7.1.3 and from the fact that $\mathbf{L}$ is fully faithful. The second isomorphism holds since $H \in \mathscr{F}$. Finally, the last isomorphism follows from the fact that $E \mathscr{F}+$ is pointed $H$-homotopy equivalent to $S^{0}$.

Proposition 7.1.4 The essential image of the functor $\mathbf{L}: \operatorname{Ho}\left(\operatorname{Sp}_{G, \mathscr{F}}^{O}\right) \rightarrow \operatorname{Ho}\left(\operatorname{Sp}_{G}^{O}\right)$ is exactly the localizing subcategory generated by $\left\{\Sigma_{+}^{\infty} G / H \mid H \in \mathscr{F}\right\}$. 
Proof The functor $\mathbf{L}$ is exact, and as we already noted, $\operatorname{Ho}\left(\operatorname{Sp}_{G, \mathscr{F}}^{O}\right)$ is generated by the set $\left\{\Sigma_{+}^{\infty} G / H \mid H \in \mathscr{F}\right\}$. Next, by Proposition 7.1.3, for any $H \in \mathscr{F}$,

$$
\mathbf{L}\left(\Sigma_{+}^{\infty} G / H\right) \cong E \mathscr{F}_{+} \wedge \Sigma_{+}^{\infty} G / H .
$$

The projection map $E \mathscr{F}_{+} \wedge \Sigma_{+}^{\infty} G / H \rightarrow \Sigma_{+}^{\infty} G / H$ is a weak equivalence in $\operatorname{Sp}_{G}^{O}$. The rest of the proof follows from the fact that $\mathbf{L}$ is full.

Next, we need the following simple lemma from category theory.

Lemma 7.1.5 Let

$$
\mathbf{L}: \mathscr{D} \rightleftarrows \mathscr{E}: \mathbf{R}
$$

be an adjunction and assume that the unit

$$
\text { id } \longrightarrow \text { RL }
$$

is an isomorphism (or, equivalently, $\mathbf{L}$ is fully faithful). Further, suppose we are given morphisms

$$
X \stackrel{\alpha}{\longrightarrow} Z \stackrel{\beta}{\longleftarrow} Y
$$

in $\mathscr{E}$ such that $X$ and $Y$ are in the essential image of $\mathbf{L}$, and that $\mathbf{R}(\alpha)$ and $\mathbf{R}(\beta)$ are isomorphisms in $\mathscr{D}$. Then there is an isomorphism $\gamma: X \stackrel{\cong}{\longrightarrow} Y$ in $\mathscr{E}$ such that the following diagram commutes:

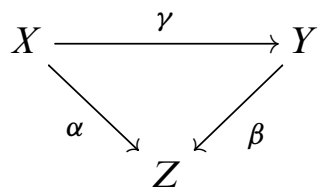

Proof One has the commutative diagram

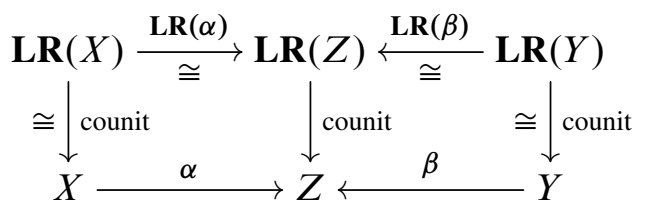

where the left and right vertical arrows are isomorphisms since $X$ and $Y$ are in the essential image of $\mathbf{L}$ and the functor $\mathbf{L}$ is fully faithful. We can choose $\gamma: X \cong Y$ to be the composite

$$
X \stackrel{\text { counit }^{-1}}{\longrightarrow} \mathbf{L R}(X) \stackrel{\mathbf{L R}(\alpha)}{\longrightarrow} \mathbf{L R}(Z) \stackrel{(\mathbf{L R}(\beta))^{-1}}{\longrightarrow} \mathbf{L R}(Y) \stackrel{\text { counit }}{\longrightarrow} Y .
$$


Corollary 7.1.6 Let $\mathscr{F}$ be a family of subgroups of $G$ and suppose $X$ and $Y$ are in the essential image of $\mathbf{L}: \operatorname{Ho}\left(\operatorname{Sp}_{G}^{O}, \mathscr{F}\right) \rightarrow \operatorname{Ho}\left(\operatorname{Sp}_{G}^{O}\right)$ (which is the localizing subcategory generated by $\left\{\Sigma_{+}^{\infty} G / H \mid H \in \mathscr{F}\right\}$ according to Proposition 7.1.4). Further assume that we have maps

$$
X \stackrel{\alpha}{\longrightarrow} Z \stackrel{\beta}{\longleftarrow} Y
$$

such that $\pi_{*}^{H} \alpha$ and $\pi_{*}^{H} \beta$ are isomorphisms for any $H \in \mathscr{F}$ (or, in other words, $\alpha$ and $\beta$ are $\mathscr{F}$-equivalences). Then there is an isomorphism $\gamma: X \stackrel{\cong}{\cong} Y$ such that the following diagram commutes:

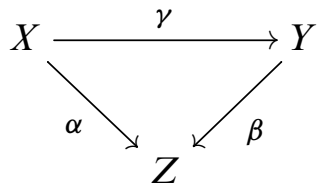

Proof We apply the previous lemma to the adjunction

$$
\mathbf{L}: \operatorname{Ho}\left(\operatorname{Sp}_{G, \mathscr{F}}^{O}\right) \rightleftarrows \operatorname{Ho}\left(\operatorname{Sp}_{G}^{O}\right): \mathbf{R},
$$

and use the isomorphism $\pi_{*}^{H} \mathbf{R}(T) \cong \pi_{*}^{H} T$ for $H \in \mathscr{F}$.

\subsection{Inductive strategy and preservation of induced classifying spaces}

Recall that we are given an exact functor of triangulated categories

$$
F: \operatorname{Ho}\left(\operatorname{Sp}_{G}^{O},{ }_{(2)}\right) \longrightarrow \operatorname{Ho}\left(\operatorname{Sp}_{G}^{O},(2)\right)
$$

that preserves arbitrary coproducts and such that

$$
F\left(\Sigma_{+}^{\infty} G / H\right)=\Sigma_{+}^{\infty} G / H, \quad H \leq G,
$$

and

$$
F(g)=g, \quad F\left(\operatorname{res}_{K}^{H}\right)=\operatorname{res}_{K}^{H}, \quad \text { and } \quad F\left(\operatorname{tr}_{K}^{H}\right)=\operatorname{tr}_{K}^{H}, \quad g \in G, K \leq H \leq G .
$$

We want to show that $F$ is an equivalence of categories. Proposition 5.1.1 implies that, in order to prove that $F$ is an equivalence, it suffices to check that, for any subgroup $H \leq G$, the map between graded endomorphism rings

$F:\left[\Sigma_{+}^{\infty} G / H, \Sigma_{+}^{\infty} G / H\right]_{*}^{G} \rightarrow\left[F\left(\Sigma_{+}^{\infty} G / H\right), F\left(\Sigma_{+}^{\infty} G / H\right)\right]_{*}^{G}=\left[\Sigma_{+}^{\infty} G / H, \Sigma_{+}^{\infty} G / H\right]_{*}^{G}$ 
is an isomorphism. Indeed, there is a commutative diagram

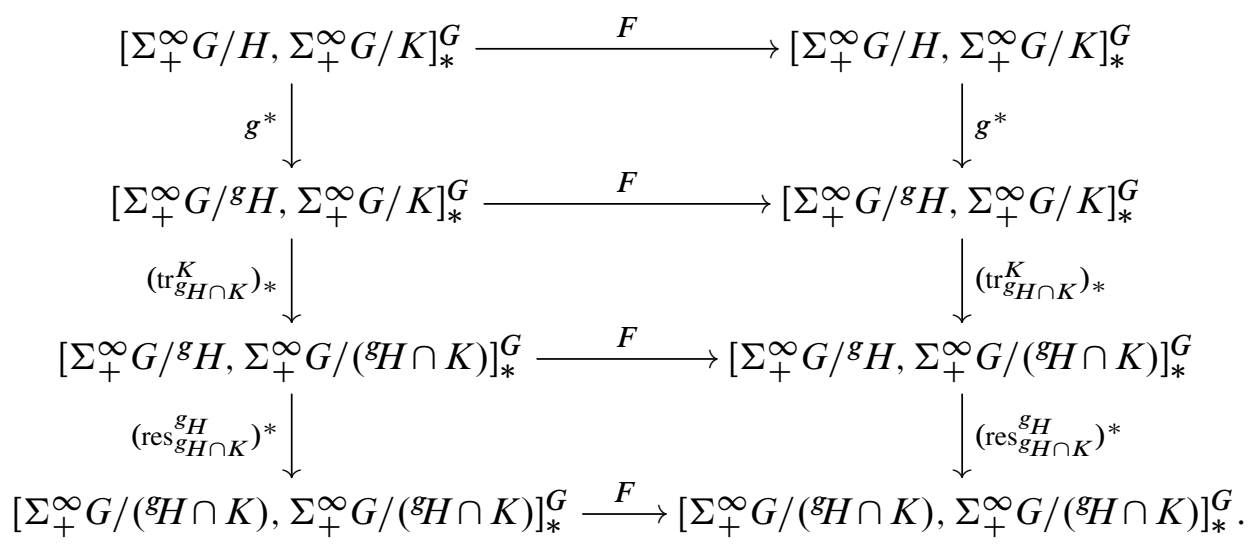

for any $g \in G$, which implies that the diagram

$\left[\Sigma_{+}^{\infty} G / H, \Sigma_{+}^{\infty} G / K\right]_{*}^{G} \stackrel{\left(\kappa_{g}\right)_{[g] \in K \backslash G / H}}{\longrightarrow} \bigoplus \quad\left[\Sigma_{+}^{\infty} G /\left({ }^{g} H \cap K\right), \Sigma_{+}^{\infty} G /\left({ }^{g} H \cap K\right)\right]_{*}^{G}$

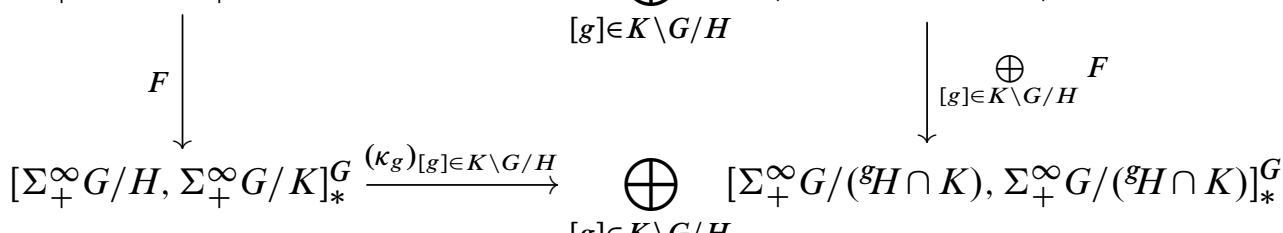
$[g] \in K \backslash G / H$

commutes. If we now assume that, for any subgroup $L \leq G$, the map

$$
F:\left[\Sigma_{+}^{\infty} G / L, \Sigma_{+}^{\infty} G / L\right]_{*}^{G} \longrightarrow\left[\Sigma_{+}^{\infty} G / L, \Sigma_{+}^{\infty} G / L\right]_{*}^{G}
$$

is an isomorphism, then the right vertical map in the latter commutative square is an isomorphism. Proposition 5.1.1 implies that the horizontal maps are injective. Hence, by a simple diagram chase, it follows that the left vertical morphism is injective as well. But now we know that, for $*=0$, the morphism

$$
F:\left[\Sigma_{+}^{\infty} G / H, \Sigma_{+}^{\infty} G / K\right]_{*}^{G} \longrightarrow\left[\Sigma_{+}^{\infty} G / H, \Sigma_{+}^{\infty} G / K\right]_{*}^{G}
$$

is the identity, and for $*>0$, it has the same finite source and target; see Subsection 2.8. Combining this with the latter injectivity result allows us to conclude that the map

$$
F:\left[\Sigma_{+}^{\infty} G / H, \Sigma_{+}^{\infty} G / K\right]_{*}^{G} \longrightarrow\left[\Sigma_{+}^{\infty} G / H, \Sigma_{+}^{\infty} G / K\right]_{*}^{G}
$$

is an isomorphism for any integer $*$. Finally, since the set $\left\{\Sigma_{+}^{\infty} G / H \mid H \leq G\right\}$ is a set of compact generators for the triangulated category $\operatorname{Ho}\left(\operatorname{Sp}_{G}^{O},(2)\right)$, Lemma 2.2.2 completes the proof of Theorem 3.1.3. 
Hence it remains to show that, for any subgroup $H \leq G$, the map between graded endomorphism rings

$F:\left[\Sigma_{+}^{\infty} G / H, \Sigma_{+}^{\infty} G / H\right]_{*}^{G} \rightarrow\left[F\left(\Sigma_{+}^{\infty} G / H\right), F\left(\Sigma_{+}^{\infty} G / H\right)\right]_{*}^{G}=\left[\Sigma_{+}^{\infty} G / H, \Sigma_{+}^{\infty} G / H\right]_{*}^{G}$

is an isomorphism. We proceed by induction on the cardinality of $H$. The induction starts with the case $H=e$. Proposition 4.2.1 tells us that the map

$$
F:\left[\Sigma_{+}^{\infty} G, \Sigma_{+}^{\infty} G\right]_{*}^{G} \longrightarrow\left[\Sigma_{+}^{\infty} G, \Sigma_{+}^{\infty} G\right]_{*}^{G}
$$

is an isomorphism, and hence the basis step is proved. The induction step follows from the next proposition, which is one of the main technical results of this paper.

Proposition 7.2.1 Let $G$ be a finite group and $H$ a subgroup of $G$. Assume that, for any subgroup $K$ of $G$ that is proper subconjugate to $H$, the map

$F:\left[\Sigma_{+}^{\infty} G / K, \Sigma_{+}^{\infty} G / K\right]_{*}^{G} \rightarrow\left[F\left(\Sigma_{+}^{\infty} G / K\right), F\left(\Sigma_{+}^{\infty} G / K\right)\right]_{*}^{G}=\left[\Sigma_{+}^{\infty} G / K, \Sigma_{+}^{\infty} G / K\right]_{*}^{G}$ is an isomorphism. Then the map

$F:\left[\Sigma_{+}^{\infty} G / H, \Sigma_{+}^{\infty} G / H\right]_{*}^{G} \rightarrow\left[F\left(\Sigma_{+}^{\infty} G / H\right), F\left(\Sigma_{+}^{\infty} G / H\right)\right]_{*}^{G}=\left[\Sigma_{+}^{\infty} G / H, \Sigma_{+}^{\infty} G / H\right]_{*}^{G}$ is an isomorphism.

Before starting to prove this proposition, one has to show that, under its assumptions, the functor $F$ preserves the object $G \ltimes_{H} \Sigma_{+}^{\infty} E \mathscr{P}[H]$. More precisely, let $\mathscr{P}[H]$ denote the family of proper subgroups of $H$. This is a family with respect to $H$ and not necessarily with respect to the whole group $G$. Next, let

$$
\text { proj: } G \ltimes_{H} \Sigma_{+}^{\infty} E \mathscr{P}[H] \longrightarrow \Sigma_{+}^{\infty} G / H
$$

be the projection (as in Subsection 6.3). The following holds:

Lemma 7.2.2 Suppose $G$ is a finite group and $H$ a subgroup of $G$. Assume that, for any subgroup $K$ of $G$ that is proper subconjugate to $H$, the map

$$
F:\left[\Sigma_{+}^{\infty} G / K, \Sigma_{+}^{\infty} G / K\right]_{*}^{G} \longrightarrow\left[\Sigma_{+}^{\infty} G / K, \Sigma_{+}^{\infty} G / K\right]_{*}^{G}
$$

is an isomorphism. Then there is an isomorphism

$$
\gamma: F\left(G \ltimes_{H} \Sigma_{+}^{\infty} E \mathscr{P}[H]\right) \stackrel{\cong}{\longrightarrow} G \ltimes_{H} \Sigma_{+}^{\infty} E \mathscr{P}[H]
$$

such that the following diagram commutes:

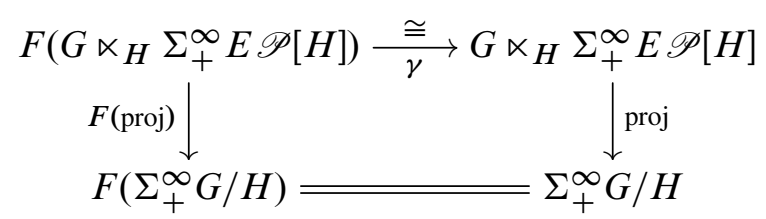


Proof Let $\mathscr{P}[H \mid G]$ denote the family of subgroups of $G$ that are proper subconjugate to $H$. By Proposition 7.1.4, the essential image of the fully faithful embedding

$$
\text { L: } \operatorname{Ho}\left(\operatorname{Sp}_{G, \mathscr{P}[H \mid G]}^{O}\right) \longrightarrow \operatorname{Ho}\left(\operatorname{Sp}_{G}^{O}\right)
$$

is the localizing subcategory generated by the set $\left\{\Sigma_{+}^{\infty} G / K \mid K \in \mathscr{P}[H \mid G]\right\}$. Obviously, the spectrum $G \ltimes_{H} \Sigma_{+}^{\infty} E \mathscr{P}[H]$ is an object of this localizing subcategory as the $H-\mathrm{CW}$ complex $E \mathscr{P}[H]$ is built out of $H$-cells of orbit type $H / K$ with $K \leq H$ and $K \neq H$. Next, since the endofunctor $F: \operatorname{Ho}\left(\operatorname{Sp}_{G}^{O}\right) \rightarrow \operatorname{Ho}\left(\operatorname{Sp}_{G}^{O}\right)$ is exact, preserves infinite coproducts and $F\left(\Sigma_{+}^{\infty} G / L\right)=\Sigma_{+}^{\infty} G / L$ for any $L \leq G$, the spectrum $F\left(G \ltimes_{H} \Sigma_{+}^{\infty} E \mathscr{P}[H]\right)$ is contained in the essential image of $\mathbf{L}: \operatorname{Ho}\left(\operatorname{Sp}_{G, \mathscr{P}[H \mid G]}^{O}\right) \rightarrow$ $\mathrm{Ho}\left(\mathrm{Sp}_{G}^{O}\right)$ as well. Hence by Corollary 7.1.6, it suffices to show the maps in the zigzag

$$
F\left(G \ltimes_{H} \Sigma_{+}^{\infty} E \mathscr{P}[H]\right) \stackrel{F(\text { proj })}{\longrightarrow} F\left(\Sigma_{+}^{\infty} G / H\right)=\Sigma_{+}^{\infty} G / H \stackrel{\text { proj }}{\longleftarrow} G \ltimes_{H} \Sigma_{+}^{\infty} E \mathscr{P}[H]
$$

are $\mathscr{P}[H \mid G]$-equivalences (which means that they induce isomorphisms on $\pi_{*}^{K}(-$ ) for any subgroup $K \in \mathscr{P}[H \mid G]$ ). First, we check that the map

$$
\text { proj: } G \ltimes_{H} \Sigma_{+}^{\infty} E \mathscr{P}[H] \longrightarrow \Sigma_{+}^{\infty} G / H
$$

is a $\mathscr{P}[H \mid G]$-equivalence. Indeed, by Corollary 6.4.3, for any $K \in \mathscr{P}[H \mid G]$, one has the following commutative diagram:

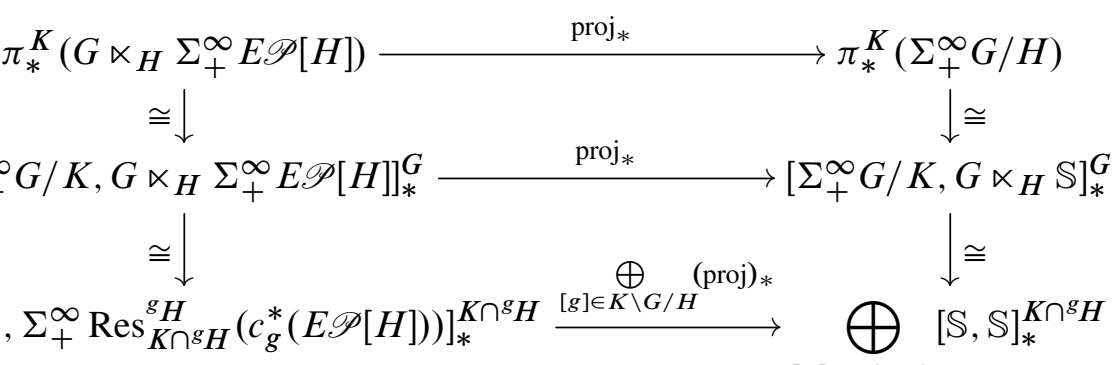
$[g] \in K \backslash G / H$ $[g] \in K \backslash G / H$

If $L$ is a subgroup of $K \cap{ }^{g} H$, then $g^{-1} L g$ is a subgroup of $H$. In fact, $g^{-1} L g$ is a proper subgroup of $H$ since $K \in \mathscr{P}[H \mid G]$. This implies that, for any subgroup $L \leq K \cap{ }^{g} H$, the space

$$
\left(\operatorname{Res}_{K \cap g_{H}}^{g_{H}}\left(c_{g}^{*}(E \mathscr{P}[H])\right)\right)^{L}=(E \mathscr{P}[H])^{g^{-1} L g}
$$

is contractible. Hence, $\operatorname{Res}_{K \cap}^{g}{ }_{H}^{g} H\left(c_{g}^{*}(E \mathscr{P}[H])\right)$ is a $\left(K \cap{ }^{g} H\right)$-contractible cofibrant $\left(K \cap{ }^{g} H\right)$-space and we see that the map

$$
\text { proj: } \Sigma_{+}^{\infty} \operatorname{Res}_{K{ }^{g}{ }^{g}{ }_{H}}\left(c_{g}^{*}(E \mathscr{P}[H])\right) \longrightarrow \mathbb{S}
$$


is a $\left(K \cap{ }^{g} H\right)$-equivalence. From this, we conclude that the lower horizontal map in the latter commutative diagram is an isomorphism. Hence the upper horizontal map is an isomorphism for any subgroup $K \in \mathscr{P}[H \mid G]$, and one concludes that the map

$$
\text { proj: } G \ltimes_{H} \Sigma_{+}^{\infty} E \mathscr{P}[H] \longrightarrow \Sigma_{+}^{\infty} G / H
$$

is a $\mathscr{P}[H \mid G]$-equivalence.

It remains to show that the morphism

$$
F\left(\text { proj): } F\left(G \ltimes_{H} \Sigma_{+}^{\infty} E \mathscr{P}[H]\right) \rightarrow F\left(\Sigma_{+}^{\infty} G / H\right)\right.
$$

is a $\mathscr{P}[H \mid G]$-equivalence as well. We first note that the assumptions imply that, for any $K \in \mathscr{P}[H \mid G]$ and any (not necessarily proper) subgroup $L \leq H$, the map

$F:\left[\Sigma_{+}^{\infty} G / K, \Sigma_{+}^{\infty} G / L\right]_{*}^{G} \longrightarrow\left[F\left(\Sigma_{+}^{\infty} G / K\right), F\left(\Sigma_{+}^{\infty} G / L\right)\right]_{*}^{G}=\left[\Sigma_{+}^{\infty} G / K, \Sigma_{+}^{\infty} G / L\right]_{*}^{G}$

is an isomorphism. Indeed, this follows from Proposition 5.1.1 as well as from the following commutative diagram:

$\left[\Sigma_{+}^{\infty} G / K, \Sigma_{+}^{\infty} G / L\right]_{*}^{G} \stackrel{\left(\kappa_{\lambda}\right)_{[\lambda] \in K \backslash G / L}}{\longrightarrow}\left[\left[\Sigma_{+}^{\infty} G /\left({ }^{\lambda} L \cap K\right), \Sigma_{+}^{\infty} G /\left({ }^{\lambda} L \cap K\right)\right]_{*}^{G}\right.$ $[\lambda] \in K \backslash G / L$

$$
\downarrow_{[\lambda] \in K \backslash G / L}^{\oplus} F
$$

$\left[\Sigma_{+}^{\infty} G / K, \Sigma_{+}^{\infty} G / L\right]_{*}^{G} \stackrel{\left(\kappa_{\lambda}\right)_{[\lambda] \in K \backslash G / L}}{\longrightarrow} \bigoplus\left[\Sigma_{+}^{\infty} G /\left({ }^{\lambda} L \cap K\right), \Sigma_{+}^{\infty} G /\left({ }^{\lambda} L \cap K\right)\right]_{*}^{G}$ $[\lambda] \in K \backslash G / L$

The right vertical map is an isomorphism since ${ }^{\lambda} L \cap K$ is proper subconjugate to $H$ for any $\lambda$. In particular, the map

$$
F:\left[\Sigma_{+}^{\infty} G / K, \Sigma_{+}^{\infty} G / H\right]_{*}^{G} \longrightarrow\left[\Sigma_{+}^{\infty} G / K, \Sigma_{+}^{\infty} G / H\right]_{*}^{G}
$$

is an isomorphism. Next, using a standard argument on triangulated categories, we see that, for any $K \in \mathscr{P}[H \mid G]$ and any $X$ from the localizing subcategory of $\mathrm{Ho}\left(\mathrm{Sp}_{G}^{O}\right)$ generated by $\left\{\Sigma_{+}^{\infty} G / L \mid L \leq H\right\}$, the map

$$
F:\left[\Sigma_{+}^{\infty} G / K, X\right]_{*}^{G} \longrightarrow\left[F\left(\Sigma_{+}^{\infty} G / K\right), F(X)\right]_{*}^{G}
$$

is an isomorphism (recall $F\left(\Sigma_{+}^{\infty} G / L\right)=\Sigma_{+}^{\infty} G / L$ for any $\left.L \leq G\right)$. As a consequence, we see that the morphism

$$
F:\left[\Sigma_{+}^{\infty} G / K, G \ltimes_{H} \Sigma_{+}^{\infty} E \mathscr{P}[H]\right]_{*}^{G} \longrightarrow\left[F\left(\Sigma_{+}^{\infty} G / K\right), F\left(G \ltimes_{H} \Sigma_{+}^{\infty} E \mathscr{P}[H]\right)\right]_{*}^{G}
$$


is an isomorphism. Finally, for any $K \in \mathscr{P}[H \mid G]$, consider the following commutative diagram:

$$
\begin{aligned}
& {\left[\Sigma_{+}^{\infty} G / K, G \ltimes_{H} \Sigma_{+}^{\infty} E \mathscr{P}[H]\right]_{*}^{G} \longrightarrow\left[\Sigma_{+}^{\infty} G / K, \Sigma_{+}^{\infty} G / H\right]_{*}^{G}} \\
& \cong \downarrow F \\
& {\left[F\left(\Sigma_{+}^{\infty} G / K\right), F\left(G \ltimes_{H} \Sigma_{+}^{\infty} E \mathscr{P}[H]\right)\right]_{*}^{G} \stackrel{F(\mathrm{proj})_{*}}{\longrightarrow}\left[F\left(\Sigma_{+}^{\infty} G / K\right), F\left(\Sigma_{+}^{\infty} G / H\right)\right]_{*}^{G}} \\
& \| \\
& {\left[\Sigma_{+}^{\infty} G / K, F\left(G \ltimes_{H} \Sigma_{+}^{\infty} E \mathscr{P}[H]\right)\right]_{*}^{G} \stackrel{F(\mathrm{proj})_{*}}{\longrightarrow}\left[\Sigma_{+}^{\infty} G / K, F\left(\Sigma_{+}^{\infty} G / H\right)\right]_{*}^{G}}
\end{aligned}
$$

As we already explained, the upper horizontal map is an isomorphism. Thus the lower horizontal map in this diagram is an isomorphism as well, and therefore, the map

$$
F\left(\text { proj): } F\left(G \ltimes_{H} \Sigma_{+}^{\infty} E \mathscr{P}[H]\right) \longrightarrow F\left(\Sigma_{+}^{\infty} G / H\right)\right.
$$

is a $\mathscr{P}[H \mid G]$-equivalence.

\subsection{Completing the proof of Theorem 3.1.3}

In this subsection, we continue the induction started in the previous subsection and prove Proposition 7.2.1. Finally, at the end, we complete the proof of Theorem 3.1.3 and hence prove the main Theorem 1.1.1.

Proof of Proposition 7.2.1 Recall (from Section 6) that $W$ denotes the Weyl group $W(H)$, and that the extension

$$
1 \longrightarrow H \stackrel{\iota}{\longrightarrow} N(H) \stackrel{\varepsilon}{\longrightarrow} W \longrightarrow 1
$$

determines the inflation functor

$$
\varepsilon^{*}: \operatorname{Ho}\left(\operatorname{Sp}_{W}^{O}\right) \longrightarrow \operatorname{Ho}\left(\operatorname{Sp}_{N(H)}^{O}\right)
$$

and the geometric fixed point functor

$$
\Phi^{H}: \operatorname{Ho}\left(\operatorname{Sp}_{N(H)}^{O}\right) \longrightarrow \operatorname{Ho}\left(\operatorname{Sp}_{W}^{O}\right) .
$$

Let $\widehat{F}: \operatorname{Ho}\left(\operatorname{Sp}_{W}^{O}\right) \longrightarrow \operatorname{Ho}\left(\operatorname{Sp}_{W}^{O}\right)$ denote the composite

$\mathrm{Ho}\left(\mathrm{Sp}_{W}^{O}\right) \stackrel{\varepsilon^{*}}{\longrightarrow} \mathrm{Ho}\left(\mathrm{Sp}_{N(H)}^{O}\right) \stackrel{G \ltimes_{N(H)}-}{\longrightarrow} \mathrm{Ho}\left(\mathrm{Sp}_{G}^{O}\right)$

$$
\stackrel{F}{\longrightarrow} \mathrm{Ho}\left(\mathrm{Sp}_{G}^{O}\right) \stackrel{\operatorname{Res}_{N(H)}^{G}}{\longrightarrow} \mathrm{Ho}\left(\operatorname{Sp}_{N(H)}^{O}\right) \stackrel{\Phi^{H}}{\longrightarrow} \mathrm{Ho}\left(\mathrm{Sp}_{W}^{O}\right)
$$


It follows from the identifications we did in Subsection 6.2 and from the properties of $F$ that the functor $\hat{F}$ is exact, preserves infinite coproducts and sends $\Sigma_{+}^{\infty} W$ to itself. Moreover, it also follows that the restriction

$$
\left.\widehat{F}\right|_{\operatorname{Ho}\left(\operatorname{Mod}-\Sigma_{+}^{\infty} W\right)}: \operatorname{Ho}\left(\operatorname{Mod}-\Sigma_{+}^{\infty} W\right) \longrightarrow \operatorname{Ho}\left(\operatorname{Mod}-\Sigma_{+}^{\infty} W\right)
$$

of $\widehat{F}$ on the localizing subcategory of $\operatorname{Ho}\left(\operatorname{Sp}_{W}^{O}\right)$ generated by $\Sigma_{+}^{\infty} W$ satisfies the assumptions of Proposition 4.2.1. Hence the map

$$
\widehat{F}:\left[\Sigma_{+}^{\infty} W, \Sigma_{+}^{\infty} W\right]_{*}^{W} \longrightarrow\left[\Sigma_{+}^{\infty} W, \Sigma_{+}^{\infty} W\right]_{*}^{W}
$$

is an isomorphism. Next, by the assumptions and Proposition 5.1.1 (like in the proof of Lemma 7.2.2), we see that for any proper subgroup $L$ of $H$, the map

$$
F:\left[\Sigma_{+}^{\infty} G / H, \Sigma_{+}^{\infty} G / L\right]_{*}^{G} \longrightarrow\left[\Sigma_{+}^{\infty} G / H, \Sigma_{+}^{\infty} G / L\right]_{*}^{G}
$$

is an isomorphism. This, using a standard argument on triangulated categories, implies that for any $X$ which is contained in the localizing subcategory of $\operatorname{Ho}\left(\operatorname{Sp}_{G}^{O}\right)$ generated by $\left\{\Sigma_{+}^{\infty} G / L \mid L \in \mathscr{P}[H]\right\}$, the map

$$
F:\left[\Sigma_{+}^{\infty} G / H, X\right]_{*}^{G} \longrightarrow\left[F\left(\Sigma_{+}^{\infty} G / H\right), F(X)\right]_{*}^{G}
$$

is an isomorphism and hence, in particular, so is the morphism

$$
F:\left[\Sigma_{+}^{\infty} G / H, G \ltimes_{H} \Sigma_{+}^{\infty} E \mathscr{P}[H]\right]_{*}^{G} \longrightarrow\left[F\left(\Sigma_{+}^{\infty} G / H\right), F\left(G \ltimes_{H} \Sigma_{+}^{\infty} E \mathscr{P}[H]\right)\right]_{*}^{G} .
$$

Finally, we have the following important commutative diagram:

$$
\begin{aligned}
& \stackrel{\left[\Sigma_{+}^{\infty} G / H,\right.}{\left.G \ltimes_{H} \Sigma_{+}^{\infty} E \mathscr{P}[H]\right]_{*}^{G}} \stackrel{\operatorname{proj}_{*}}{\longrightarrow} \begin{array}{c}
{\left[\Sigma_{+}^{\infty} G / H,\right.} \\
\left.\Sigma_{+}^{\infty} G / H\right]_{*}^{G}
\end{array} \stackrel{G \ltimes_{N(H)} \varepsilon^{*}}{\longleftarrow}\left[\Sigma_{+}^{\infty} W, \Sigma_{+}^{\infty} W\right]_{*}^{W} \\
& \cong \downarrow F \\
& \begin{array}{l}
{\left[F\left(\Sigma_{+}^{\infty} G / H\right),\right.} \\
\left.F\left(G \ltimes_{H} \Sigma_{+}^{\infty} E \mathscr{P}[H]\right)\right]_{*}^{G} \stackrel{F(\mathrm{proj})_{*}}{\longrightarrow} \underset{F\left(\Sigma_{+}^{\infty} G / H\right),}{\left.\left[\Sigma_{+}^{\infty} G / H\right)\right]_{*}^{G}} \stackrel{\Phi_{\mathrm{res}}^{H}}{\longrightarrow}\left[\widehat{F}\left(\Sigma_{+}^{\infty} W\right), \widehat{F}\left(\Sigma_{+}^{\infty} W\right)\right]_{*}^{W}
\end{array} \\
& \cong \downarrow
\end{aligned}
$$

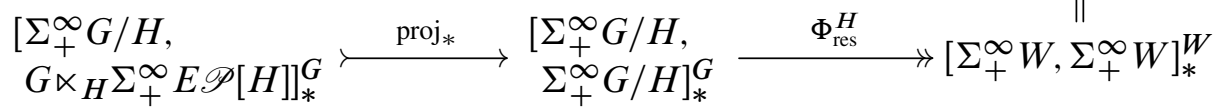

Lemma 7.2.2 implies that the lower left square commutes, and the lower left vertical map is an isomorphism. Other squares commute by definitions. Further, according to Proposition 6.3.2, the lower row in this diagram is a short exact sequence, and hence so is the middle one. 
Now a simple diagram chase shows that the map

$F:\left[\Sigma_{+}^{\infty} G / H, \Sigma_{+}^{\infty} G / H\right]_{*}^{G} \rightarrow\left[F\left(\Sigma_{+}^{\infty} G / H\right), F\left(\Sigma_{+}^{\infty} G / H\right)\right]_{*}^{G}=\left[\Sigma_{+}^{\infty} G / H, \Sigma_{+}^{\infty} G / H\right]_{*}^{G}$

is an isomorphism. Indeed, assume that $*>0$ (the case $*=0$ is obvious by the assumptions on $F$ ). Then the latter map has the same finite source and target, and hence it suffices to show that it is surjective. Fix $*>0$ and take any $\alpha \in\left[F\left(\Sigma_{+}^{\infty} G / H\right), F\left(\Sigma_{+}^{\infty} G / H\right)\right]_{*}^{G}$. Since the map

$$
\widehat{F}:\left[\Sigma_{+}^{\infty} W, \Sigma_{+}^{\infty} W\right]_{*}^{W} \longrightarrow\left[\widehat{F}\left(\Sigma_{+}^{\infty} W\right), \widehat{F}\left(\Sigma_{+}^{\infty} W\right)\right]_{*}^{W}
$$

is an isomorphism, there exists $\beta \in\left[\Sigma_{+}^{\infty} W, \Sigma_{+}^{\infty} W\right]_{*}^{W}$ such that

$$
\widehat{F}(\beta)=\Phi_{\text {res }}^{H}(\alpha) \text {. }
$$

By definition of the functor $\widehat{F}$, the element

$$
F\left(G \ltimes_{N(H)} \varepsilon^{*}(\beta)\right)-\alpha \in\left[F\left(\Sigma_{+}^{\infty} G / H\right), F\left(\Sigma_{+}^{\infty} G / H\right)\right]_{*}^{G}
$$

is in the kernel of

$$
\Phi_{\text {res }}^{H}:\left[F\left(\Sigma_{+}^{\infty} G / H\right), F\left(\Sigma_{+}^{\infty} G / H\right)\right]_{*}^{G} \longrightarrow\left[\widehat{F}\left(\Sigma_{+}^{\infty} W\right), \widehat{F}\left(\Sigma_{+}^{\infty} W\right)\right]_{*}^{W} .
$$

But the kernel of this map is contained in the image of

$$
F:\left[\Sigma_{+}^{\infty} G / H, \Sigma_{+}^{\infty} G / H\right]_{*}^{G} \longrightarrow\left[F\left(\Sigma_{+}^{\infty} G / H\right), F\left(\Sigma_{+}^{\infty} G / H\right)\right]_{*}^{G}
$$

since the middle row in the commutative diagram above is exact and the upper left vertical map is an isomorphism. Consequently, $F\left(G \ltimes_{N(H)} \varepsilon^{*}(\beta)\right)-\alpha$ is in the image of $F$, and this completes the proof.

Proof of Theorem 3.1.3 Recall that our aim is to show that for any subgroup $H \leq G$, the map

$$
F:\left[\Sigma_{+}^{\infty} G / H, \Sigma_{+}^{\infty} G / H\right]_{*}^{G} \longrightarrow\left[\Sigma_{+}^{\infty} G / H, \Sigma_{+}^{\infty} G / H\right]_{*}^{G}
$$

is an isomorphism. We have already shown (in Subsection 7.2) that this, together with Proposition 5.1.1, would imply that the map

$$
F:\left[\Sigma_{+}^{\infty} G / L, \Sigma_{+}^{\infty} G / L^{\prime}\right]_{*}^{G} \longrightarrow\left[\Sigma_{+}^{\infty} G / L, \Sigma_{+}^{\infty} G / L^{\prime}\right]_{*}^{G}
$$

is an isomorphism for any integer $*$ and subgroups $L, L^{\prime} \leq G$. Finally, by Lemma 2.2.2, Theorem 3.1.3 will follow.

The strategy to prove the last claim about the graded endomorphisms was indicated in Subsection 7.2. We proceed by induction on the cardinality of $H$. The induction basis follows from Proposition 4.2.1 as we already explained. Now suppose $n>1$, 
and assume that the claim holds for all subgroups of $G$ with cardinality less than or equal to $n-1$. Let $H$ be any subgroup of $G$ that has cardinality equal to $n$. Then, by the induction assumption, for any subgroup $K$ which is proper subconjugate to $H$, the map

$$
F:\left[\Sigma_{+}^{\infty} G / K, \Sigma_{+}^{\infty} G / K\right]_{*}^{G} \longrightarrow\left[\Sigma_{+}^{\infty} G / K, \Sigma_{+}^{\infty} G / K\right]_{*}^{G}
$$

is an isomorphism. Proposition 7.2.1 now implies that

$$
F:\left[\Sigma_{+}^{\infty} G / H, \Sigma_{+}^{\infty} G / H\right]_{*}^{G} \longrightarrow\left[\Sigma_{+}^{\infty} G / H, \Sigma_{+}^{\infty} G / H\right]_{*}^{G}
$$

is an isomorphism and this completes the proof of the claim.

Acknowledgements This paper is based on my $\mathrm{PhD}$ thesis at the University of Bonn. I would like to thank my advisor Stefan Schwede for suggesting the project, for introducing me to the beautiful world of equivariant stable homotopy theory, and for his help and encouragement throughout the way. I owe special thanks to Justin Noel for many hours of helpful mathematical discussions and for his help in simplifying some of the proofs in this paper. I am also indebted to Markus Hausmann, Kristian Moi and Karol Szumiło for reading earlier drafts of the paper and for giving me valuable comments and suggestions. Further, I benefited from discussions with David Barnes, John Greenlees, Michael Hill, Michael Hopkins, Peter May, Lennart Meier, Constanze Roitzheim, Steffen Sagave, Brooke Shipley, Markus Szymik and many other people.

This research was supported by the Deutsche Forschungsgemeinschaft Graduiertenkolleg 1150 "Homotopy and Cohomology". During the final revision of the paper, the author was supported by the Danish National Research Foundation through the Centre for Symmetry and Deformation (DNRF92).

\section{References}

[1] J F Adams, On the non-existence of elements of Hopf invariant one, Ann. of Math. 72 (1960) 20-104

[2] V Angeltveit, Topological Hochschild homology and cohomology of $A_{\infty}$ ring spectra, Geom. Topol. 12 (2008) 987-1032 MR2403804

[3] D Barnes, C Roitzheim, Rational equivariant rigidity, from: "An alpine expedition through algebraic topology”, (C Ausoni, K Hess, B Johnson, W Lück, J Scherer, editors), Contemp. Math. 617, Amer. Math. Soc. (2014) 13-30 MR3243391

[4] H-J Baues, F Muro, Toda brackets and cup-one squares for ring spectra, Comm. Algebra 37 (2009) 56-82 MR2482810

[5] A J Blumberg, Continuous functors as a model for the equivariant stable homotopy category, Algebr. Geom. Topol. 6 (2006) 2257-2295 MR2286026 
[6] J M Boardman, On stable homotopy theory and some applications, $\mathrm{PhD}$ thesis, University of Cambridge (1964)

[7] A K Bousfield, On the telescopic homotopy theory of spaces, Trans. Amer. Math. Soc. 353 (2001) 2391-2426 MR1814075

[8] A K Bousfield, E M Friedlander, Homotopy theory of $\Gamma$-spaces, spectra, and bisimplicial sets, from: "Geometric applications of homotopy theory, II", (M G Barratt, ME Mahowald, editors), Lecture Notes in Math. 658, Springer, Berlin (1978) 80-130 MR513569

[9] J M Cohen, The decomposition of stable homotopy, Ann. of Math. 87 (1968) 305-320

[10] T tom Dieck, Transformation groups, de Gruyter Studies in Mathematics 8, de Gruyter, Berlin (1987)

[11] D Dugger, B Shipley, K-theory and derived equivalences, Duke Math. J. 124 (2004) 587-617 MR2085176

[12] D Dugger, B Shipley, A curious example of triangulated-equivalent model categories which are not Quillen equivalent, Algebr. Geom. Topol. 9 (2009) 135-166 MR2482071

[13] W G Dwyer, J Spaliński, Homotopy theories and model categories, from: "Handbook of algebraic topology”, (I M James, editor), North-Holland, Amsterdam (1995) 73-126 MR1361887

[14] A D Elmendorf, Systems of fixed point sets, Trans. Amer. Math. Soc. 277 (1983) 275-284 MR690052

[15] A D Elmendorf, I Kriz, M A Mandell, J P May, Rings, modules, and algebras in stable homotopy theory, Mathematical Surveys and Monographs 47, Amer. Math. Soc. (1997) MR1417719

[16] S I Gelfand, Y I Manin, Methods of homological algebra, 2nd edition, Springer, Berlin (2003) MR1950475

[17] P G Goerss, J F Jardine, Simplicial homotopy theory, Progress in Mathematics 174, Birkhäuser, Basel (1999) MR1711612

[18] J P C Greenlees, J P May, Generalized Tate cohomology, Mem. Amer. Math. Soc. 543, Amer. Math. Soc., Providence, RI (1995) MR1230773

[19] B Guillou, J P May, Enriched model categories and presheaf categories, preprint (2013) arXiv:1110.3567

[20] M Hausmann, G-symmetric spectra, semistability and the multiplicative norm, preprint (2014) arXiv:1411.2290

[21] M A Hill, MJ Hopkins, D C Ravenel, On the nonexistence of elements of Kervaire invariant one, Ann. of Math. (2) 184 (2016) 1-262 MR3505179

[22] PS Hirschhorn, Model categories and their localizations, Mathematical Surveys and Monographs 99, Amer. Math. Soc., Providence, RI (2003) MR1944041 
[23] M Hovey, Model categories, Mathematical Surveys and Monographs 63, Amer. Math. Soc., Providence, RI (1999) MR1650134

[24] M Hovey, B Shipley, J Smith, Symmetric spectra, J. Amer. Math. Soc. 13 (2000) 149-208 MR1695653

[25] K Hutschenreuter, On rigidity of the ring spectra $P_{m} \mathbb{S}_{(p)}$ and $k o, \mathrm{PhD}$ thesis, Universität Bonn (2012) Available at http://hss.ulb.uni-bonn.de/2012/2974/ 2974.htm

[26] S Illman, The equivariant triangulation theorem for actions of compact Lie groups, Math. Ann. 262 (1983) 487-501 MR696520

[27] D M Kan, Semisimplicial spectra, Illinois J. Math. 7 (1963) 463-478

[28] G M Kelly, Basic concepts of enriched category theory, London Mathematical Society Lecture Note Series 64, Cambridge Univ. Press (1982)

[29] L G Lewis, Jr, The category of Mackey functors for a compact Lie group, from: "Group representations: cohomology, group actions and topology", (A Adem, J Carlson, S Priddy, P Webb, editors), Proc. Sympos. Pure Math. 63, Amer. Math. Soc., Providence, RI (1998) 301-354 MR1603183

[30] L G Lewis, Jr, J P May, M Steinberger, J E McClure, Equivariant stable homotopy theory, Lecture Notes in Mathematics 1213, Springer, Berlin (1986) MR866482

[31] M Lydakis, Simplicial functors and stable homotopy theory, unpublished manuscript (1998) Available at http://www.math.uoc.gr/ mlydakis/papers/sf.pdf

[32] M A Mandell, Equivariant symmetric spectra, from: "Homotopy theory: relations with algebraic geometry, group cohomology, and algebraic $K$-theory”, (P Goerss, S Priddy, editors), Contemp. Math. 346, Amer. Math. Soc., Providence, RI (2004) 399-452 MR2066508

[33] M A Mandell, J P May, Equivariant orthogonal spectra and S-modules, Mem. Amer. Math. Soc. 755, Amer. Math. Soc., Providence, RI (2002) MR1922205

[34] M A Mandell, J P May, S Schwede, B Shipley, Model categories of diagram spectra, Proc. London Math. Soc. 82 (2001) 441-512 MR1806878

[35] J P May, Equivariant homotopy and cohomology theory, CBMS Regional Conference Series in Mathematics 91, Amer. Math. Soc., Providence, RI (1996) MR1413302

[36] J P May, The Wirthmüller isomorphism revisited, Theory Appl. Categ. 11 (2003) 132-142 MR1988073

[37] A Neeman, Triangulated categories, Annals of Mathematics Studies 148, Princeton Univ. Press (2001) MR1812507

[38] I Patchkoria, On the algebraic classification of module spectra, Algebr. Geom. Topol. 12 (2012) 2329-2388 MR3020210

[39] D G Quillen, Homotopical algebra, Lecture Notes Math. 43, Springer, Berlin (1967) 
[40] C Roitzheim, Rigidity and exotic models for the K-local stable homotopy category, Geom. Topol. 11 (2007) 1855-1886 MR2350470

[41] M Schlichting, A note on K-theory and triangulated categories, Invent. Math. 150 (2002) 111-116 MR1930883

[42] S Schwede, The stable homotopy category has a unique model at the prime 2, Adv. Math. 164 (2001) 24-40 MR1870511

[43] S Schwede, The stable homotopy category is rigid, Ann. of Math. 166 (2007) 837-863 MR2373374

[44] S Schwede, Algebraic versus topological triangulated categories, from: "Triangulated categories", (Cambridge, editor), London Math. Soc. Lecture Note Ser. 375, Cambridge Univ. Press (2010) 389-407 MR2681714

[45] S Schwede, Lectures on equivariant stable homotopy theory, lecture notes, Universität Bonn (2014) Available at http://www.math.uni-bonn.de/ schwede/ equivariant.pdf

[46] S Schwede, B Shipley, A uniqueness theorem for stable homotopy theory, Math. Z. 239 (2002) 803-828 MR1902062

[47] S Schwede, B Shipley, Stable model categories are categories of modules, Topology 42 (2003) 103-153 MR1928647

[48] M Stolz, Equivariant structures on smash powers of commutative ring spectra, $\mathrm{PhD}$ thesis, University of Bergen (2011) Available at https://www.math.rochester.edu/ people/faculty/doug/otherpapers/mstolz.pdf

[49] H Toda, Composition methods in homotopy groups of spheres, Annals of Mathematics Studies 49, Princeton Univ. Press (1962)

[50] C A Weibel, An introduction to homological algebra, Cambridge Studies in Advanced Mathematics 38, Cambridge Univ. Press (1994) MR1269324

Department of Mathematical Sciences, University of Copenhagen

Universitetsparken 5, DK-2100 Copenhagen, Denmark

irakli.p@math.ku.dk

Received: 19 June 2015 
